\title{
A Multivariate Analysis of Crash and Naturalistic Driving Data in Relation to Highway Factors
}




\section{TRANSPORTATION RESEARCH BOARD 2013 EXECUTIVE COMMITTEE*}

\section{OFFICERS}

CHAIR: Deborah H. Butler, Executive Vice President, Planning, and CIO, Norfolk Southern Corporation, Norfolk, Virginia

VICE CHAIR: Kirk T. Steudle, Director, Michigan Department of Transportation, Lansing

EXECUTIVE DIRECTOR: Robert E. Skinner, Jr., Transportation Research Board

\section{MEMBERS}

Victoria A. Arroyo, Executive Director, Georgetown Climate Center, and Visiting Professor, Georgetown University Law Center, Washington, D.C. Scott E. Bennett, Director, Arkansas State Highway and Transportation Department, Little Rock

William A. V. Clark, Professor of Geography (emeritus) and Professor of Statistics (emeritus), Department of Geography, University of California, Los Angeles

James M. Crites, Executive Vice President of Operations, Dallas-Fort Worth International Airport, Texas

Malcolm Dougherty, Director, California Department of Transportation, Sacramento

John S. Halikowski, Director, Arizona Department of Transportation, Phoenix

Michael W. Hancock, Secretary, Kentucky Transportation Cabinet, Frankfort

Susan Hanson, Distinguished University Professor Emerita, School of Geography, Clark University, Worcester, Massachusetts

Steve Heminger, Executive Director, Metropolitan Transportation Commission, Oakland

Chris T. Hendrickson, Duquesne Light Professor of Engineering, Carnegie Mellon University, Pittsburgh, Pennsylvania

Jeffrey D. Holt, Managing Director, Bank of Montreal Capital Markets, and Chairman, Utah Transportation Commission, Huntsville, Utah

Gary P. LaGrange, President and CEO, Port of New Orleans, Louisiana

Michael P. Lewis, Director, Rhode Island Department of Transportation, Providence

Joan McDonald, Commissioner, New York State Department of Transportation, Albany

Donald A. Osterberg, Senior Vice President, Safety and Security, Schneider National, Inc., Green Bay, Wisconsin

Steve Palmer, Vice President of Transportation, Lowe's Companies, Inc., Mooresville, North Carolina

Sandra Rosenbloom, Director, Innovation in Infrastructure, The Urban Institute, Washington, D.C. (Past Chair, 2012)

Henry G. (Gerry) Schwartz, Jr., Chairman (retired), Jacobs/Sverdrup Civil, Inc., St. Louis, Missouri

Kumares C. Sinha, Olson Distinguished Professor of Civil Engineering, Purdue University, West Lafayette, Indiana

Daniel Sperling, Professor of Civil Engineering and Environmental Science and Policy; Director, Institute of Transportation Studies; University of California, Davis

Gary C. Thomas, President and Executive Director, Dallas Area Rapid Transit, Dallas, Texas

Phillip A. Washington, General Manager, Regional Transportation District, Denver, Colorado

\section{EX OFFICIO MEMBERS}

Rebecca M. Brewster, President and COO, American Transportation Research Institute, Marietta, Georgia Anne S. Ferro, Administrator, Federal Motor Carrier Safety Administration, U.S. Department of Transportation LeRoy Gishi, Chief, Division of Transportation, Bureau of Indian Affairs, U.S. Department of the Interior, Washington, D.C. John T. Gray II, Senior Vice President, Policy and Economics, Association of American Railroads, Washington, D.C.

Michael P. Huerta, Administrator, Federal Aviation Administration, U.S. Department of Transportation

David T. Matsuda, Administrator, Maritime Administration, U.S. Department of Transportation

Michael P. Melaniphy, President and CEO, American Public Transportation Association, Washington, D.C.

Victor M. Mendez, Administrator, Federal Highway Administration, U.S. Department of Transportation

Robert J. Papp (Adm., U.S. Coast Guard), Commandant, U.S. Coast Guard, U.S. Department of Homeland Security

Lucy Phillips Priddy, Research Civil Engineer, U.S. Army Corps of Engineers, Vicksburg, Mississippi, and Chair, TRB Young Members Council Cynthia L. Quarterman, Administrator, Pipeline and Hazardous Materials Safety Administration, U.S. Department of Transportation

Peter M. Rogoff, Administrator, Federal Transit Administration, U.S. Department of Transportation

David L. Strickland, Administrator, National Highway Traffic Safety Administration, U.S. Department of Transportation

Joseph C. Szabo, Administrator, Federal Railroad Administration, U.S. Department of Transportation

Polly Trottenberg, Under Secretary for Policy, U.S. Department of Transportation

Robert L. Van Antwerp (Lt. General, U.S. Army), Chief of Engineers and Commanding General, U.S. Army Corps of Engineers, Washington, D.C. Barry R. Wallerstein, Executive Officer, South Coast Air Quality Management District, Diamond Bar, California

Gregory D. Winfree, Acting Administrator, Research and Innovative Technology Administration, U.S. Department of Transportation Frederick G. (Bud) Wright, Executive Director, American Association of State Highway and Transportation Officials, Washington, D.C.

\footnotetext{
${ }^{\star}$ Membership as of April 2013.
} 


\title{
A Multivariate Analysis of Crash and Naturalistic Driving Data in Relation to Highway Factors
}

T. J. Gordon, L. P. Kostyniuk, P. E. Green, M. A. Barnes, D. F. Blower,

S. E. Bogard, A. D. Blankespoor, AND D. J. LeBlanC

University of Michigan Transportation Research Institute

\author{
B. R. Cannon and S. B. McLaughlin \\ Virginia Tech Transportation Institute
}




\section{Subscriber Categories}

Highways

Safety and Human Factors 


\section{The Second Strategic Highway Research Program}

America's highway system is critical to meeting the mobility and economic needs of local communities, regions, and the nation. Developments in research and technology_such as advanced materials, communications technology, new data collection technologies, and human factors science-offer a new opportunity to improve the safety and reliability of this important national resource. Breakthrough resolution of significant transportation problems, however, requires concentrated resources over a short time frame. Reflecting this need, the second Strategic Highway Research Program (SHRP 2) has an intense, large-scale focus, integrates multiple fields of research and technology, and is fundamentally different from the broad, mission-oriented, discipline-based research programs that have been the mainstay of the highway research industry for half a century.

The need for SHRP 2 was identified in TRB Special Report 260: Strategic Highway Research: Saving Lives, Reducing Congestion, Improving Quality of Life, published in 2001 and based on a study sponsored by Congress through the Transportation Equity Act for the 21st Century (TEA-21). SHRP 2, modeled after the first Strategic Highway Research Program, is a focused, timeconstrained, management-driven program designed to complement existing highway research programs. SHRP 2 focuses on applied research in four areas: Safety, to prevent or reduce the severity of highway crashes by understanding driver behavior; Renewal, to address the aging infrastructure through rapid design and construction methods that cause minimal disruptions and produce lasting facilities; Reliability, to reduce congestion through incident reduction, management, response, and mitigation; and Capacity, to integrate mobility, economic, environmental, and community needs in the planning and designing of new transportation capacity.

SHRP 2 was authorized in August 2005 as part of the Safe, Accountable, Flexible, Efficient Transportation Equity Act: A Legacy for Users (SAFETEA-LU). The program is managed by the Transportation Research Board (TRB) on behalf of the National Research Council (NRC). SHRP 2 is conducted under a memorandum of understanding among the American Association of State Highway and Transportation Officials (AASHTO), the Federal Highway Administration (FHWA), and the National Academy of Sciences, parent organization of TRB and NRC. The program provides for competitive, merit-based selection of research contractors; independent research project oversight; and dissemination of research results.
SHRP 2 Report S2-S01C-RW-1

ISBN: 978-0-309-12890-2

(c) 2013 National Academy of Sciences. All rights reserved.

\section{Copyright Information}

Authors herein are responsible for the authenticity of their materials and for obtaining written permissions from publishers or persons who own the copyright to any previously published or copyrighted material used herein.

The second Strategic Highway Research Program grants permission to reproduce material in this publication for classroom and not-for-profit purposes. Permission is given with the understanding that none of the material will be used to imply TRB, AASHTO, or FHWA endorsement of a particular product, method, or practice. It is expected that those reproducing material in this document for educational and not-for-profit purposes will give appropriate acknowledgment of the source of any reprinted or reproduced material. For other uses of the material, request permission from SHRP 2.

Note: SHRP 2 report numbers convey the program, focus area, project number, and publication format. Report numbers ending in " $w$ " are published as web documents only.

\section{Notice}

The project that is the subject of this report was a part of the second Strategic Highway Research Program, conducted by the Transportation Research Board with the approval of the Governing Board of the National Research Council.

The members of the technical committee selected to monitor this project and to review this report were chosen for their special competencies and with regard for appropriate balance. The report was reviewed by the technical committee and accepted for publication according to procedures established and overseen by the Transportation Research Board and approved by the Governing Board of the National Research Council.

The opinions and conclusions expressed or implied in this report are those of the researchers who performed the research and are not necessarily those of the Transportation Research Board, the National Research Council, or the program sponsors.

The Transportation Research Board of the National Academies, the National Research Council, and the sponsors of the second Strategic Highway Research Program do not endorse products or manufacturers. Trade or manufacturers' names appear herein solely because they are considered essential to the object of the report.

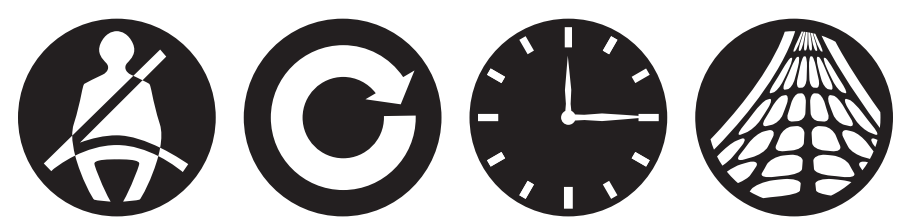

\section{SHRP 2 Reports}

Available by subscription and through the TRB online bookstore: www.TRB.org/bookstore

Contact the TRB Business Office:

202-334-3213

More information about SHRP 2:

www.TRB.org/SHRP2 


\section{THE NATIONAL ACADEMIES Advisers to the Nation on Science, Engineering, and Medicine}

The National Academy of Sciences is a private, nonprofit, self-perpetuating society of distinguished scholars engaged in scientific and engineering research, dedicated to the furtherance of science and technology and to their use for the general welfare. On the authority of the charter granted to it by Congress in 1863, the Academy has a mandate that requires it to advise the federal government on scientific and technical matters. Dr. Ralph J. Cicerone is president of the National Academy of Sciences.

The National Academy of Engineering was established in 1964, under the charter of the National Academy of Sciences, as a parallel organization of outstanding engineers. It is autonomous in its administration and in the selection of its members, sharing with the National Academy of Sciences the responsibility for advising the federal government. The National Academy of Engineering also sponsors engineering programs aimed at meeting national needs, encourages education and research, and recognizes the superior achievements of engineers. Dr. C. D. (Dan) Mote, Jr., is president of the National Academy of Engineering.

The Institute of Medicine was established in 1970 by the National Academy of Sciences to secure the services of eminent members of appropriate professions in the examination of policy matters pertaining to the health of the public. The Institute acts under the responsibility given to the National Academy of Sciences by its congressional charter to be an adviser to the federal government and, on its own initiative, to identify issues of medical care, research, and education. Dr. Harvey V. Fineberg is president of the Institute of Medicine.

The National Research Council was organized by the National Academy of Sciences in 1916 to associate the broad community of science and technology with the Academy's purposes of furthering knowledge and advising the federal government. Functioning in accordance with general policies determined by the Academy, the Council has become the principal operating agency of both the National Academy of Sciences and the National Academy of Engineering in providing services to the government, the public, and the scientific and engineering communities. The Council is administered jointly by both Academies and the Institute of Medicine. Dr. Ralph J. Cicerone and Dr. C. D. (Dan) Mote, Jr., are chair and vice chair, respectively, of the National Research Council.

The Transportation Research Board is one of six major divisions of the National Research Council. The mission of the Transportation Research Board is to provide leadership in transportation innovation and progress through research and information exchange, conducted within a setting that is objective, interdisciplinary, and multimodal. The Board's varied activities annually engage about 7,000 engineers, scientists, and other transportation researchers and practitioners from the public and private sectors and academia, all of whom contribute their expertise in the public interest. The program is supported by state transportation departments, federal agencies including the component administrations of the U.S. Department of Transportation, and other organizations and individuals interested in the development of transportation. Www.TRB.org

wWw.national-academies.org 


\section{SHRP 2 STAFF}

Ann M. Brach, Director

Stephen J. Andrle, Deputy Director

Neil J. Pedersen, Deputy Director, Implementation and Communications

James Bryant, Senior Program Officer, Renewal

Kenneth Campbell, Chief Program Officer, Safety

JoAnn Coleman, Senior Program Assistant, Capacity and Reliability

Eduardo Cusicanqui, Financial Officer

Walter Diewald, Senior Program Officer, Safety

Jerry DiMaggio, Implementation Coordinator

Shantia Douglas, Senior Financial Assistant

Charles Fay, Senior Program Officer, Safety

Carol Ford, Senior Program Assistant, Renewal and Safety

Elizabeth Forney, Assistant Editor

Jo Allen Gause, Senior Program Officer, Capacity

Rosalind Gomes, Accounting/Financial Assistant

Abdelmename Hedhli, Visiting Professional

James Hedlund, Special Consultant, Safety Coordination

Alyssa Hernandez, Reports Coordinator

Ralph Hessian, Special Consultant, Capacity and Reliability

Andy Horosko, Special Consultant, Safety Field Data Collection

William Hyman, Senior Program Officer, Reliability

Michael Marazzi, Senior Editorial Assistant

Linda Mason, Communications Officer

Reena Mathews, Senior Program Officer, Capacity and Reliability

Matthew Miller, Program Officer, Capacity and Reliability

Michael Miller, Senior Program Assistant, Capacity and Reliability

David Plazak, Senior Program Officer, Capacity

Onno Tool, Visiting Professional

Dean Trackman, Managing Editor

Connie Woldu, Administrative Coordinator

Patrick Zelinski, Communications/Media Associate

\section{ACKNOWLEDGMENTS}

This work was sponsored by the Federal Highway Administration in cooperation with the American Association of State Highway and Transportation Officials. It was conducted in the second Strategic Highway Research Program (SHRP 2), which is administered by the Transportation Research Board of the National Academies. The project was managed by Walter Diewald, Senior Program Officer for Safety.

The research reported herein was performed by the University of Michigan Transportation Research Institute (UMTRI) in collaboration with the Virginia Tech Transportation Institute (VTTI). Timothy Gordon was the project director and principal investigator. The authors acknowledge the important role of Michael Hagan and other members of the UMTRI Engineering Research Division, who collected and managed the naturalistic driving data used in this project. Additional thanks are due to the Michigan Department of Transportation for providing the Highway Performance Management System data for the public road system and to the Michigan Center for Geographic Information for providing the digital public road framework map. 


\section{F O R E W R D \\ Walter Diewald, PhD, SHRP 2 Senior Program Officer, Safety}

A large component of the safety research undertaken in the second Strategic Highway Research Program (SHRP 2) is aimed at reducing the injuries and fatalities that result from highway crashes. Through a naturalistic driving study (NDS) involving more than 3,000 volunteer drivers, SHRP 2 expects to learn more about how individual driver behavior interacts with vehicle and roadway characteristics. In anticipation of the large volume of data to be collected during the NDS, several projects were conducted to demonstrate that it is possible to use existing data from previous naturalistic driving studies and data from other sources to further the understanding of the risk factors associated with road crashes. More specifically, the four S01 projects, titled Development of Analysis Methods Using Recent Data, examined the statistical relationship between surrogate measures of collisions (conflicts, critical incidents, near collisions, and roadside encroachment) and actual collisions. This report presents the results of one of these projects, undertaken by the University of Michigan Transportation Research Institute.

This report documents the second phase of a two-phase project under SHRP 2 Safety Project S01C. A primary part of this work involved conducting a multivariate analysis of crash and naturalistic driving data in relation to highway factors. A geographic information system (GIS) framework was used as the basis for fusing multiple information sources to analyze road departure crash risk. A major goal was to use this method to support formulation and validation of crash surrogates.

Two analytical models developed in the study focus on the statistical relationship between surrogate measures of crashes and actual crashes and on the formulation of exposure-based risk measures using surrogate measures. The first approach is an extension of the traditional univariate response model for crashes to a model that treats crashes and crash surrogates as a bivariate response variable, incorporates a correlation structure between them, and can be extended to a Bayesian model. The second approach is based on extreme value theory and estimates the probability of events that are more extreme than any that have been observed. The surrogate measures examined ranged from relatively simple measures based on lane position and time to the crossing of a lane boundary to more complex measures, such as a driver's adjustment of the vehicle yaw angle to match that of the road.

The report also describes three exploratory studies that illustrate the value of the geospatial approach taken. The first study examined the application of spatial statistics to the problem of determining if concentrations of crashes were really "hot spots" or if they could be considered simply random groupings. The second study compared surrogate event rates in episodes of driving while on and off using a cell phone. The comparison was made for the same driver for the same conditions. The third study compared the yaw rate error of drivers through areas of lane widening and locations with uniform lane widths. The results of each exploratory study suggest that the combination of naturalistic, crash, and highway data provides a rich data resource for many types of research. 
This report provides valuable background information to highway safety analysts seeking to use the data that will be made available from the SHRP 2 NDS. The beneficiaries of such research will be the nation's highway users as vehicle design and road design, as well as highway traffic control, are improved through the analytical use of the data. 
1 Executive Summary

3 CHAPTER 1 Introduction

5 CHAPTER 2 Research Questions

5 Research Hypotheses

6 Broad Research Questions

6 Specific Safety Research Questions

7 Data Quality and Validation

8 CHAPTER 3 Data Sources

$8 \quad$ UMTRI Naturalistic Driving Data

8 Highway Data

$9 \quad$ Crash Data

10 Analysis Data

CHAPTER 4 Surrogates for Road Departure Crashes

Overview of Available Surrogates

Specific Surrogates Used for Analysis the Analysis of Rates for Crashes and Crash Surrogates

Traditional Analysis of Crash Data

Seemingly Unrelated Regression Model

Poisson Log-Linear Models Estimated by Weighted Least Squares

Bayesian SUR for Log-Linear Models

SUR Model Application and Results

CHAPTER 6 Statistical Analysis: An Approach Using Extreme Value Theory Extreme Value Analysis

27 CHAPTER 7 Yaw Rate Error

35
CHAPTER 8 Orthogonal Studies

Road Departure Crash Spatial Analysis

Cell Phone Use

Road Boundary Change and Yaw Rate Error

CHAPTER 9 Transferability to Other Data Sources

Data Used for Testing

Method

NDD through VDOT Hot Spots

Summary 


\section{References}

54 Appendix A. Literature Review: Crash Rates and Highway, Environmental, and Driver Factors

59 Appendix B. Road Departure Crashes by Environmental and Driver Conditions

61 Appendix C. Data Tables

63 Appendix D. Determination of Yaw Rate Error from Vehicle-Based Measurements 


\section{Executive Summary}

The research presented in this report deals with the joint statistical analysis of crash data and naturalistic driving data (NDD), referenced in a common spatial framework. The aim is to provide a validated quantitative link between detailed measurements of naturalistic driving behavior, road departure crashes, and road segment characteristics. No such link exists, and therefore the goal was to develop appropriate analysis methods capable of associating crash risk with quantitative metrics ("crash surrogates") available from NDD. In this process, geographic information system (GIS) tools and other analysis tools were used. When applied to results of the future SHRP 2 naturalistic driving study (NDS), the methods should provide quantitative relationships between driving and crash risk, provide validated surrogates for these types of crashes, and develop new understanding of risk factors, which can be used to improve highway safety. This work is exploratory in nature and uses preexisting driving data from southeastern Michigan to develop initial statistical models and formulate appropriate metrics.

The study is based on the idea that the underlying mechanisms leading to single-vehicle road departure crashes are the same as those that create variations in normal driving, especially those involving "disturbed" lane-keeping control. More specifically, the study formulates the road departure crash problem according to the following set of hypotheses:

1. Single-vehicle road departure crashes occur only under conditions of disturbed control.

2. NDD contain measurable episodes of disturbed control.

3. Crash surrogates exist and are based on a combination of objective measures of disturbed control (from onboard sensors), highway geometric factors, and off-highway factors (environmental factors).

4. Crash surrogates can be related to actual crashes.

Numerous research questions were formulated around this theme. The first of these questions relates to the measurement of disturbed control in naturalistic driving. A set of 14 metrics was formulated, all of them relating to the quality of lane-keeping control or the attention of the driver, or both. Since the driving data used did not contain objective variables for driver head or eye movement, not all possibilities were explored in detail. Statistical analysis was in fact restricted to three candidate metrics:

- Lateral deviation (LDEV): The vehicle lateral deviation from the center of the lane exceeds a threshold based on an overall frequency distribution obtained from the driving data.

- Lane departure warning (LDW): The onboard lane departure system used in the driving study gave an alert to the driver.

- Time to edge crossing (TTEC): The estimated time to departing the paved surface, on the basis of lane position and shoulder width, is less than a certain threshold (again based on an overall frequency distribution). 
A fourth candidate metric, the yaw rate error (YRE), was formulated to overcome some of the limitations of the three metrics listed above, but no statistical analysis was carried out on the YRE in this research.

Further research questions were formulated around the variables and methods for performing a joint analysis of the crash data and driving data. As a starting point, a common measure of exposure was found in the form of normalized road segment traversals. The same road segment definitions were used for both data sets, though segments with zero exposure in the NDD were excluded from the study. A unified approach was adopted for the combined analysis of crash rates and surrogate events. The seemingly unrelated regression (SUR) method was adopted because it allows for the use of common explanatory variables in the two data sets and is flexible enough to include additional explanatory variables that are not available in both. This is an important property for future analysis in SHRP 2, in which driver attention variables may be included in the explanatory set for NDD (and no such quantitative information is usually available for crashes). Bayesian estimation was used to determine posterior distributions of the SUR model parameters and also to estimate relative risk (RR) between surrogate and crashes. The posterior distributions of the logarithm of the relative risk (log RR) provided a set of validity tests of the surrogate used. The difference in log RR between crash and surrogate events should be zero for any particular comparison, meaning that zero should be contained within an associated confidence interval. On the one hand, it was found that the simplest surrogate, LDEV, did not satisfy this criterion in the case of a curve/no-curve comparison, and therefore LDEV was not seen as acceptable for use as a crash surrogate. On the other hand, the corresponding log RR distributions for LDW and TTEC did satisfy this criterion. This analysis was not exhaustive, and was conducted as an exemplar of the method. In the future it will be important to increase the number of explanatory variables (including driver attention variables, if available) and apply multiple $\log$ RR comparisons to prioritize the wider range of metrics for lane-keeping control.

Since TTEC was found to be a reasonable candidate crash surrogate, its distribution of extremes was applied to the prediction of road departure frequencies for a single example road segment. By using extreme value theory and annual average daily traffic (AADT) counts, it was possible to estimate the number of road departures. An estimate of 12 road departures per year was obtained, compared to the actual crash number of 1.8 per year (police-reported, single-vehicle road departure crashes, averaged over a 5-year period). Since not every road departure is expected to result in a crash, this sample result is considered plausible at least. The crucial point is that a validated surrogate was needed for this type of analysis, and the surrogate needed be based on an underlying continuous variable.

Overall, this exploratory study has demonstrated the use of the SUR analysis method for the combined analysis of crash data and naturalistic driving data. The approach provides a way to assess crash risk in a common framework and to validate or invalidate candidate surrogates. More detailed analysis of individual sites can be carried out by using extreme value theory, though it is important that surrogates be continuous and display the same RR as measured crash data. Although only a small number of surrogates were analyzed, the study demonstrates the importance of surrogate choice, and a new metric — the YRE— has been defined and proposed for use in future statistical analysis. When YRE is applied to data from the future SHRP 2 NDS, the increased statistical power resulting from the much larger data set will provide more definitive conclusions about surrogate validity and factors influencing overall crash risk. 


\section{CHAPTER 1 \\ Introduction}

This study focuses on a new challenge and a new opportunity in highway safety research. In the upcoming SHRP 2 naturalistic driving study (NDS), a large new database will be created with the potential to provide entirely new information about risk factors and highway crashes. While smaller data sets of a similar nature have been created in earlier work-especially at the University of Michigan Transportation Institute (UMTRI) (LeBlanc et al. 2006) and the Virginia Tech Transportation Institute (VTTI) (Dingus et al. 2006) - the efficient analysis of the SHRP 2 data will require the development of new and innovative methods. Although the SHRP 2 database will be the largest of its kind, the number of actual crashes seen in the study is likely to be small. Therefore, as part of the overall risk analysis, the formulation and validation of surrogates are major goals.

The present study uses smaller existing data sets as testing ground for the statistical analysis of candidate surrogates. The major focus is on highway factors and the codependence of both crash events and surrogate events on these factors. The analysis to be presented relies on the integration of several sources of data: from naturalistic driving (as will be provided by the SHRP 2 Safety project), from historical crash data, and from databases of highway characteristics. These diverse data sets are related by spatial coincidence ("same highway segment," generalizing to "same segment properties") and in particular via reference to underlying road map data. This analysis approach comes under the heading of geographic information systems (GISs), where different layers of information are related via a suitable map-referencing system. Thus, the goal of this study is to develop a GIS-based analysis of crashes and crash surrogates related to highway variables to address many of the priority SHRP 2 Safety questions. The particular crash problem addressed in this study is that of road departure crashes.

Road departure crash rates depend on multiple factors, principally those associated with human behavior and highway/ traffic conditions. Traditional analysis of crash databases cannot determine the influence of human behavior in any great detail, so the "missing information" is to be developed from naturalistic driving studies. All approaches explored in this study include some mapping or common reference for associating naturalistic driving with the occurrence of crashes, and define surrogates that typify physical mechanisms that lead to road departure crashes. Many possible events or conditions can be proposed as surrogates for crashes. The surrogates can be discrete events or continuous conditions that result in a crash in the extreme, or in a noncrash event that is necessary for crash occurrence. In this study the research team focused on developing surrogates based on measures of lane-keeping control performance. Those measures started with relatively simple ones based on lane position and time to the crossing of a lane boundary but included more complex measures, such as a driver's adjustment of the yaw angle of the vehicle to match that of the road.

Two analytical methods developed in this study focused on the statistical relationship between surrogate measures of crashes and actual crashes and on formulation of exposurebased risk measures using the surrogate measures. The first approach is an extension of the traditional univariate response model for crashes to a model that treats crashes and crash surrogates as a bivariate response variable, incorporates a correlation structure between them, and can be extended to a Bayesian model. The second approach is based on extreme value theory and estimates the probability of events that are more extreme than any that have been observed.

The spatially linked data also offer opportunities to examine driving behavior from different perspectives. A set of three small exploratory studies that were orthogonal to the main thrust of the project were also conducted. The first study examined the application of spatial statistics to the problem of determining if concentrations of crashes were really "hot spots" or if they could just be considered to be random groupings. The second study compared surrogate event rates in episodes of driving while on and off a cell phone. The comparison was made for the same drivers under similar conditions. The third study 
compared the YRE of drivers through areas of lane widening and locations with uniform lane widths.

Transferability of methods developed in this study was in part demonstrated by researchers from VTTI, using VTTI's own naturalistic driving data (NDD) and highway and crash information from the state of Virginia.

This report begins with a summary of hypotheses and research questions, from fundamental research hypotheses to detailed technical questions designed to validate the methods in Chapter 2. Data sources and the development of the spatially linked data used in this study are described in Chapter 3. Chapter 4 discusses crash surrogate measures. The statistical analysis methods follow with the bivariate response and Bayesian update model reported in Chapter 5 and the extreme value model in Chapter 6. Full statistical modeling is demonstrated in these sections for a set of the simpler lane-keeping surrogate measures. Chapter 7 focuses on the feasibility of defining and computing a surrogate based on control-oriented driving performance. The orthogonal studies are summarized in Chapter 8 . The feasibility of transferring methods developed in this study for building spatially linked GIS databases by other researchers, with other data, in other areas is demonstrated in Chapter 9. Conclusions and implications for analysis of data from larger studies based on more extensive data sets, in particular those from the SHRP 2 Safety program, are discussed in Chapter 10. Supporting literature review and technical details are in the appendices. 


\section{CHAPTER 2 Research Questions}

For decades crashes have been studied as discrete events focusing on the circumstances of the crash event. This type of analysis is exemplified in the review of Appendix A, and has been used to identify the characteristics of highway features associated with higher crash experience; other factors such as traffic volumes, driver characteristics, land use, and the environmental conditions were also needed to explain or describe crash events. Furthermore, cross-sectional analyses of crash events did not address circumstances leading to a crash.

Advances in vehicle instrumentation technology have made it possible to collect longitudinal naturalistic data about the vehicle, driver, and roadway, accumulating information about events preceding a crash, if a crash occurs. However, crashes are rare events, and there are conditions in which a crash, while likely, does not occur. Thus a crash can be considered as a high-probability outcome, given a set of conditions, and validated crash surrogates could be used to identify these conditions. Assuming that no driver intentionally crashes, it follows that crashes occur when there is loss of situational control leading to a damaging impact, and hence that surrogates are related in some way to disturbances of the driving control function. Also, it should be possible to identify the disturbance of control from NDD. The research team also expects that crashes are related to crash surrogates in an objective way that the team seeks to determine.

"Control" is defined here as the effectiveness of tactical and operational aspects of the driving task (i.e., acquiring and tracking reference information for speed and steering adjustment). "Disturbed control" is any interruption or delay in the process of perception (seeing lane boundary or other relevant features that determine the required path), recognition (what are the relevant objects that are relevant to speed and steering control?), judgment/decision (of required steering, throttle pedal, or brake pedal) or action (apply corrections) in the driving task. Disturbed control is not expected to be the same as poor lane keeping. It is quite common in NDD to see lane excursions, such as "cutting a curve" or use of the shoulder, in which the driver appears fully aware of the action and is simply not tracking within the lane markings. One might argue that these excursions still represent poor control (i.e., they do not conform to the transportation researcher's expectations), but if the driver decides to manipulate the reference conditions used for steering control (essentially a tactical decision to, for example, cut the curve) and follows that action with accuracy and predictability, then at least at the operational level, the control loop is effective. The risk of such behavior clearly depends on the skill and awareness of the driver.

Because the number of factors associated with vehicle crashes increase significantly if more than one vehicle is involved, this research examines only the single-vehicle road departure crashes (i.e., crashes involving only one vehicle in which the first harmful event occurs off the roadway). Thus, the research team tentatively expects that crash surrogates are related in some way to the disturbance of the control function of the driving task, and that it is possible to identify various types of disturbance of control from naturalistic driving data. The research team also expects that crashes are related to crash surrogates. These general considerations are now formalized as research hypotheses as follows.

\section{Research Hypotheses}

The research hypotheses are as follows:

1. Single-vehicle road departure crashes occur only under conditions of disturbed control.

2. Naturalistic driving data contain measurable episodes of disturbed control.

3. Crash surrogates exist and are based on a combination of objective measures of disturbed control (from onboard sensors), highway geometric factors, and off-highway factors (environmental factors).

4. Crash surrogates can be related to actual crashes. 
When conditions leading to single-vehicle crashes are considered, the research team expects that in many cases the disturbance to the control function might be measurable over an extended period of time; for example, a drowsy driving surrogate would only emerge as significant over time as lane-control dysfunction was found to be persistent, compared to what might be seen in a short period of distraction. The key seems to be that surrogacy is an indicator of extremes in a uniform process that includes crashes at the limits, and therefore a road departure surrogate includes the crucial element that it measures how the driving control loop is disturbed and is not simply being manipulated by the driver. While this is a useful guiding principle for the definition of surrogates, it is a matter for analysis and verification of how well such surrogates are matched to actual crash data.

The intention has been to focus on those questions most directly related to the hypotheses of disturbed control, surrogacy, and relationships between data. With this focus, research questions can be posed at many levels from broad general questions down to very specific direct technical questions. The research team focused on three levels: the first level was a restatement of the research hypotheses, the second level was specific questions of safety research, and the third level was data quality and validation.

\section{Broad Research Questions}

The research questions are summarized as follows:

1. Do single-vehicle road departure crashes occur only under conditions of disturbed control?

2. Do naturalistic driving data contain measurable episodes of disturbed control?

3. Do objective measures of disturbed control from naturalistic driving data, together with highway geometric factors, off-highway factors, and environmental factors, satisfy criteria for crash surrogate (i.e., are they related to actual crashes)?

\section{Specific Safety Research Questions}

Specific research questions are broken down into four subtypes: measuring disturbed control, relating driving performance to geometric features and road departure crash frequencies, statistics, and driver factors.

\section{Measuring Disturbed Control}

1. What measures exist in naturalistic driving data that directly measure disturbed control?

2. Are vehicle kinematic measures sufficient to identify disturbed control for risk measures in single-vehicle road departure crashes?
3. Are other driving control metrics necessary (in addition to vehicle kinematic measures) to identify disturbed control?

\section{Relating Driving Performance to Geometric Features and Road Departure Crash Frequencies}

4. Are there measures of driving control performance in existing field operational test (FOT) data that depend on highway factors in a way that is consistent with singlevehicle road departure crash frequencies?

5. Are there specific highway features that are associated with single-vehicle road departure crashes and specific driving control performance measures? (Possible candidates are isolated horizontal curves, sharp horizontal curves, sequences of horizontal curves, and combinations of horizontal and vertical curves.)

6. Can roadside factors (e.g., locations of poles, trees, bridge abutments, and side slopes) be coupled to naturalistic driving data?

7. Does the coupling of roadside factors to naturalistic driving data improve correlation with actual crashes?

8. Can general descriptors of roadside environments be used in this coupling (e.g., tree density and proportion of side slope steeper than 4 to 1 ), or do we have to be more specific about location of roadside obstacles?

\section{Statistics}

9. What statistical tests are available to determine if the measures of driving control performance in naturalistic data and single-vehicle crashes depend on geometric features in a consistent way?

10. Can satisfactory crash risk predictions be made on the basis of vehicle/driver/highway information available from naturalistic driving (e.g., via extreme value theory), or do additional roadside and environmental factors need to be introduced?

\section{Driver Factors}

11. Is the pattern of driving control performance different for the same driver when distracted versus not distracted (e.g., on a cell phone or not on a cell phone)?

12. Can various driver states (e.g., drowsy, aggressive, distracted, engaged) be identified from naturalistic driving data?

13. Can driving control performance for various states be categorized more simply (i.e., good and bad, or risky and nonrisky)?

14. Is there a difference in the driving control performance of good and bad drivers (or risky and nonrisky drivers) at locations with geometric features associated with high single-vehicle crash frequency? 


\section{Data Quality and Validation}

A number of lower-level research questions are related to crosschecking and data quality:

1. What kinematic measures of driving control performance are available in the available naturalistic driving data, and what are the levels of accuracy in those measures?

2. What spatially referenced crash and highway data exist in the regions where the driving took place, and what gaps exist in the data?

3. Can the analysis of data in southeastern Michigan be applied or recreated in another region (e.g., Virginia)? 


\section{СнАРтE 3 Data Sources}

Data for this study were developed from a naturalistic driving data (NDD) resource obtained in a field operational test (FOT) conducted in southeastern Michigan and spatially joined with highway information and crash data from the same region. The following section describes the data sources and the development of the databases used in this study.

\section{UMTRI Naturalistic Driving Data}

NDD are data collected in vehicle studies using a sample of nonprofessional drivers driving test vehicles in pursuit of their normal everyday activities. Test vehicles are instrumented with a broad range of sensors to monitor the vehicle systems, driver, roadway, and environment. A data acquisition system on the vehicle collects the data, which are later transmitted to a relational database and subsequently retrieved by researchers. The raw data are supplemented by derived measures, and other relevant information (e.g., solar elevation angle). In the past decade, several FOTs have used naturalistic driving studies in the evaluation of advanced vehicle-based safety technology.

The NDD used in this study came from an FOT conducted to collect evidence of the interaction of lay drivers with a combined lane departure technology and curve speed warning system that has been termed the road departure crash warning (RDCW) system (LeBlanc et al. 2006). The program was led by the University of Michigan Transportation Research Institute (UMTRI) under a cooperative agreement with the U.S. Department of Transportation. The RDCW systems were developed and integrated by Visteon Corporation and AssistWare in preparation for the FOT. The FOT involved exposing a fleet of 11 RDCW-equipped Nissan Altima cars to 10 months of naturalistic driving. The 78 test participants were lay drivers from southeastern Michigan, randomly selected and recruited from Michigan driver licensing records, who drove these cars as their personal vehicles for several weeks. There were 9,582 trips in the RDCW FOT. Those trips covered 133,290 km $(82,773 \mathrm{mi})$ and took $2,487 \mathrm{~h}$. The RDCW FOT was expressly intended to study road departure crash warning, so that the sensors and instrumentation were ideally suited to study lane-keeping behavior.

Data gathered by using UMTRI's data acquisition system included more than 400 data signals. Among them were video samples of the forward driving scene and driver's face; differential Global Positioning System (GPS) time and position; lane tracking, including boundary type (solid, dashed); forward and side radar returns; distance to lane edge; available maneuvering distance; vehicle velocity; yaw, pitch, and roll; and sensor data on lights and windshield wipers. The data file (raw vehicle data and derived tables) is approximately 250 gigabytes in size and is stored in a SQL server database. The data from the RDCW FOT will be referred to in the rest of this document as the UMTRI NDD.

\section{Highway Data}

The major data source for highway information in this study was the enhanced Highway Performance Monitoring System (HPMS) data for the state of Michigan in 2005. The HPMS is a national-level highway information system that includes data on the extent, condition, performance, use, and operating characteristics of the nation's highways. Limited information on travel and paved miles is included in summary form for the lowest functional systems. The HPMS database contains administrative and extent of system information on all public roads, descriptive information in a mix of universe and sample data for the arterial and collector functional systems, and area-wide summary information for urbanized, small urban, and rural areas. The road system is divided into individual segments. Information about the type of road and rural/urban designation is available for all road segments. The number of through lanes is available for all segments on all road types but minor collectors and local streets. Geometric information (e.g., curves, grades, shoulder and median types and widths) and traffic information (e.g., speed limits, peak 
capacity) are available only for a sample of segments. Traffic volume information (AADT) is available for the sampled segments and also for segments that lie within special areas sampled for air quality monitoring. Each road segment in HPMS has a unique identifier in a linear referencing system (LRS), which allows the segment to be located spatially and joined to geospatial databases.

\section{Crash Data}

Michigan police-reported crash data were used to identify a set of road departure crashes for analysis. Michigan crash data were selected because they provide the best opportunity to link together crash data, roadway geometric and exposure data, and NDD from an FOT. Both the National Automotive Sampling System General Estimates System (NASS GES, or simply GES) and the NASS Crashworthiness Data System (CDS) were considered for the analysis. However, the roadway information in GES is severely limited, and it is not possible to link in data from other files (such as HPMS) because the file is well scrubbed to ensure that specific crashes cannot be identified. Crash locations within the NASS CDS also cannot be identified, which prevents linking the crashes to sources of additional data about the roadway geometry. The Michigan data also provide an example of data sources that will ideally be available for the large naturalistic driving field study. Michigan geo-locates (identifies the crash location by using latitude and longitude coordinates) virtually all crashes. HPMS files on specific roadways are also available and these data can be linked with the crash locations using the latitude and longitude coordinates. The HPMS files include AADT information so that crash frequencies at specific locations may be normalized by a measure of exposure. Finally, FOT data are available for a set of counties in southeastern Michigan and provide information about driving behavior through specific locations.

Five years of Michigan crash data (2001 to 2005) were used in the analysis, including records for almost 2.2 million crash involvements of passenger cars. The records were compiled from information recorded by police officers on State of Michigan Traffic Crash Report forms (UD-10). The form is optically scanned and data to populate the crash file variables are captured from the scan. Crashes are geolocated from the description of the crash location entered by the reporting officer.

The Michigan crash data have limitations that apply to all police-reported data. Certain items are probably recorded with reasonable accuracy and completeness, such as time of day, road type, roadway alignment, vehicle type, weather, and so on. Other more transient conditions are inherently more difficult to identify accurately, such as driver fatigue and distraction. Certain important pieces of data such as travel speed, lane position, roadway radius of curvature, and superelevation are not recorded at all. Moreover, the system of quality control on the data is not rigorous. The crash reports are supposed to be reviewed for accuracy by supervisors at the enforcement agency before being submitted to the state. But once a report is scanned, the only check is to ensure that the scanner accurately captured what was entered on the form, not whether the data were accurate in the first place.

It is important to recognize the limitations in the Michigan crash data. However, in practice, the limitations are shared with other crash data files and, on balance, the ability to link the data with the HPMS files and the availability of the NDD for the same areas as the crashes make for a very powerful combination for the analysis.

The crucial data element in the Michigan crash data is the location information. Previous experience with the location information for a project on signal optimization in Michigan has shown that the accuracy of the location information is good (Green and Blower 2007). The top image in Figure 3.1 shows an aerial photo of an intersection and the bottom is a pin map of crashes at that intersection, using a map generated by the geographic information system (GIS) package Maptitude. The longitude and latitude coordinates are accurate enough to discriminate between the different directions of travel on the two roads. After crash locations were reviewed for 130 intersections in southeastern Michigan, few were found to be inaccurate in a gross way (e.g., the location entered on the UD-10 indicated that the crash was not at the intersection specified by the longitude and latitude coordinates). Moreover,

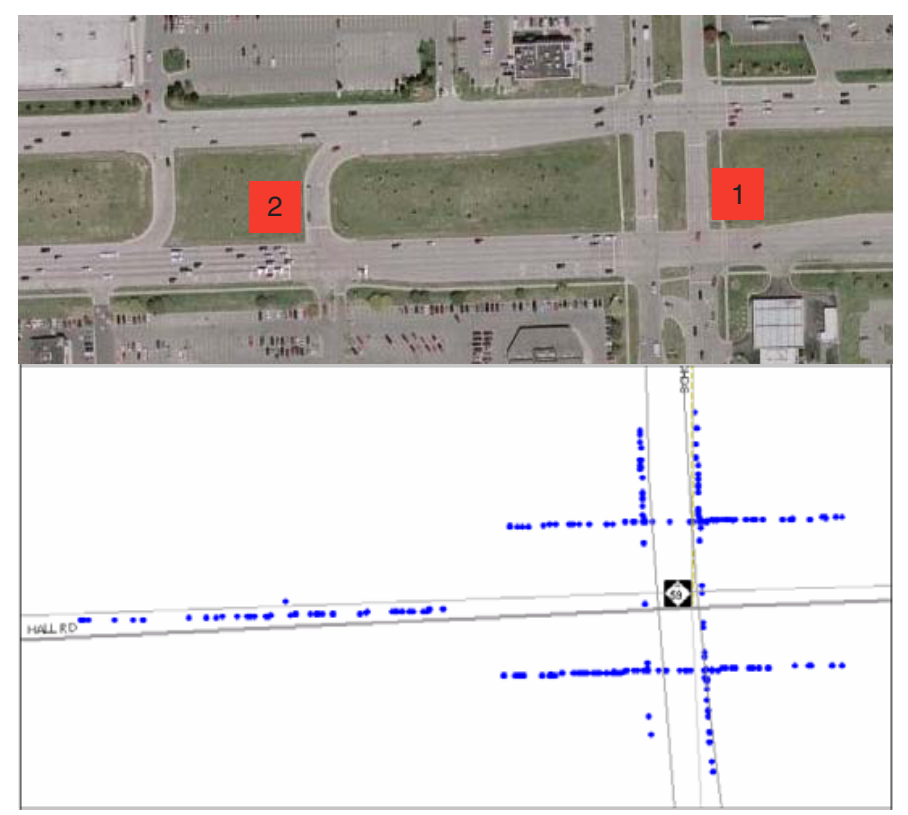

Figure 3.1. Crashes geographically located at Hall Road (M-59) and Schoenherr Road and at Hall Road and the eastbound crossover west of Schoenherr Road. 
missing data rates for location were low. Only about $2.5 \%$ of the crashes could not be located.

Road departure crashes are identified from a set of events variables. The variable records "what happened" in the crash, and include both harmful events (such as collisions) as well as nonharmful events (e.g., crossing the centerline or running off the road). Events include collisions with specific types of fixed and nonfixed objects, rollovers, roadway departures, loss of control, crossing the centerline or median, and reentering the roadway. This system in principle permits quite a complex series of events to be captured. Up to four events may be coded, though in practice only one event is coded in about $83.8 \%$ of cases. For the purposes of identifying road departure crashes, cases were flagged as roadway departure if a collision with a fixed object or run-off-road event occurred before a collision with a motor vehicle or a nonmotorist. In other words, any time a vehicle went off the road before a collision with an on-road vehicle or a nonmotorist, the case was counted as a road departure crash. Thus, road departure crashes may include on-road collisions with motor vehicles, as long as a roadway departure occurred before the collision.

The crash data include a large sample of road departure crashes for the analysis. The Michigan crash files for 2001 through 2005 include records for almost 2.2 million vehicles involved in a traffic crash, of which 192,512 records involved a roadway departure before any harmful event. The UMTRI NDD were collected in eight counties in southeastern Michigan: Lenawee, Livingston, Macomb, Monroe, Oakland, St. Clair, Washtenaw, and Wayne. Restricting the roadway departure crashes to those counties produced 73,135 involvements. Longitude and latitude were missing for 1,827 crashes (2.5\%), leaving 71,308 road departure crash involvements for analysis.
The road departure crashes in the sample are more severe than other crash types. About $0.6 \%$ of the road departure crash involvements included a fatality, compared with $0.2 \%$ of other crash involvements. About $27 \%$ included one or more injuries, compared with $23.3 \%$ of other involvements. The distributions of these road departure crashes by environmental and driver conditions are shown in Appendix B.

\section{Analysis Data}

The UMTRI NDD were spatially joined to the highway and crash data from eight counties in southeastern Michigan by using GIS software tools from ArcMap Version 9.2 from the Environmental Systems Research Institute, Inc. (Esri). A spatial base map of Michigan from the Michigan Center for Geographic Information (CGI) of the Michigan Department of Information Technology provided the key layer for the GIS tools. CGI also provided the research team with a digital map for all public roads in Michigan. The data for each road include jurisdiction, a physical reference number, and the road's functional class among other descriptors. The project used Version 6 of the base map, which represents some $8,765 \mathrm{mi}$ in southeastern Michigan. An illustration showing the four layers used to join the UMTRI data sources is shown in Figure 3.2.

In addition, CGI provided the project with ortho-imagery (aerial maps) for the southeastern Michigan region. Spatial data layered on the aerial images helped to identify pavement markings, roadside obstacles, and other road features not available via spatial data sets.

The database for subsequent analyses was developed from the spatially joined data. Only HPMS-defined segments that were also in the NDD were included. Because traffic volume

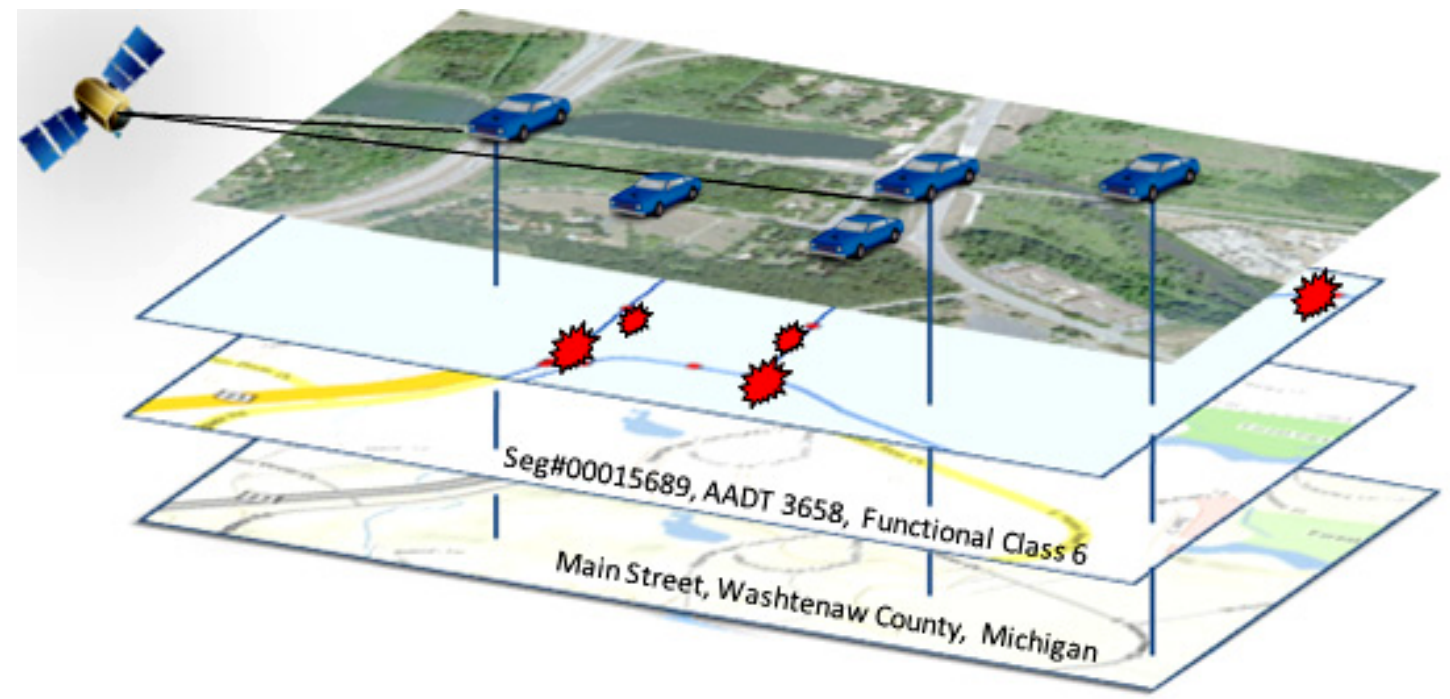

Layer (4) UMTRI Naturalistic driving data from FOT Fleets with Lat/Long positions

Layer (3) SE Michigan Road Departure Crashes (2001-05) Lat/Long

Layer (2) Michigan 2005 HPMS Data Table

Layer (1) State of Michigan public roads base map

Sources: Google Earth and MapQuest Transportation Display.

Figure 3.2. IIlustration showing the spatial layers of the data used in the UMTRI analysis. 
information was needed for exposure, HPMS segments without AADT information were excluded. Examination of the segments without volume information showed them to be minor collectors and local streets.

HPMS segments are not directional, that is, the data are for both directions of travel if the road is two-way. Because the direction of a curve and the direction of road departure are relevant in the team's analyses of road departures, the road segments in the team's analysis data are the HPMS road segments plus the direction of travel. While it is possible that the close spatial proximity of the opposite sides of the same segment of road could cause unmodeled correlations (which might be analyzed in future studies), the two travel directions were only considered to be correlated via the coincidence of the explanatory highway variables used.

Thus, the research team's basic unit of analysis was the directional road segment for which traffic volume information was provided, that had been traversed by at least one of the drivers in the NDS. There were 9,526 direction road segments in the analysis database.

Of the 71,308 road departure crashes in the eight counties of southeastern Michigan that were recorded in the Michigan crash data file from 2001 to 2005, 21,340 crashes were on the traversed directional segments in the team's analysis database. Of those crashes, 7,562 vehicles departed the road to the right and 4,372 vehicles departed to the left; in 9,406 cases, the direction of the departure was not known.

Selected attributes from the HPMS database were spatially joined to the analysis road segments. Road type information was taken directly from the functional classification of the road segment in the HPMS database. The rural/urban designation was also taken directly from the HPMS codes. Information on types and widths of shoulders was taken directly from the HPMS data, if available for the segment. Otherwise, the shoulder variables were assigned the median value obtained from the HPMS sample road segments of the same functional class, with the same number of through lanes, in the same county.

Horizontal curves in sample HPMS segments are classified into six ranges of degrees of curvature, and the total length of curves in each of these categories is reported in the HPMS database. This form of information was not useful for the research team's analyses because there was no simple and meaningful way to summarize the curve information for the segment, and also there was no credible way to impute curve information for the segments not in the HPMS sample. Because only road segments that had been traversed by an instrumented vehicle are included in the analysis database, information about the vehicle's path can be used to obtain additional roadway information. The team found that it was possible to obtain the degree of curvature and length of horizontal curves from the vehicles' path and yaw rate information. However, the procedure was labor-intensive and time-consuming. For example, drivers do not follow the same path through a curve, drivers turn off onto driveways, or a long horizontal curve appears as a series of short curves interspersed with tangent sections. While this clearly was a way of obtaining curve information, the team did not spend time and effort to develop a more efficient algorithm because there still was the problem of describing the curves if there was more than one curve in the segment.

It was clear from this experience that a different way of defining road segments for future studies is needed; for example, dividing the roadway into segments so that there is only one horizontal curve in a segment, or in a way that each horizontal curve is a unique segment, would have advantages over the use of HPMS-defined segments. For the purposes of this study, the team used the yaw rate in the NDD for the segment and defined a variable indicating the presence or absence of at least one curve in the roadway segment.

Exposure measures for each segment were also developed. Because the team used 5 years of crash data, the exposure for crashes was based on the volume of vehicles entering the segment over 5 years, as well as the segment length. The exposure for surrogate events was based on the number of traversals of instrumented vehicles and the segment length. These exposure measures are to be included in the statistical modeling, and this is considered further in Chapter 5.

The team was not able to code roadside information into its analysis database. Although the ortho-imagery provided visual information on the roadside, this information was not in a form that could be used for formal analysis. Another option would be to conduct labor-intensive manual coding of the roadside, but this option was not considered feasible. This data limitation precluded the team's ability to address research questions concerned directly with roadside features. The team recognizes that methods do exist for distinguishing between features that are found in the roadside (e.g., spectral analysis) and sees this possibility as something that could be explored in future work. 


\section{онАРтE⿱}

\section{Surrogates for Road Departure Crashes}

\section{Overview of Available Surrogates}

In Chapter 5, the statistical analysis of candidate surrogates will assume a basic form of discrete (Boolean) events. Counts are made of both crashes and surrogate events over road segments, and statistical models are developed for event rates per unit exposure, and hence relate these models to highway variables. In this way surrogate (crash) events are evaluated for overall fidelity to actual crashes, model parameters are derived, and relative risks are estimated. In Chapter 6, extreme value theory is applied to a surrogate in the form of a continuous variable. Both types of surrogates are useful and continuous surrogates may easily be converted to Boolean form via a threshold shown in Equation 4.1:

$X= \begin{cases}1 & \text { if } x>a \\ 0 & \text { otherwise }\end{cases}$

where $x$ is the continuous surrogate and $X$ is the resulting Boolean one. Typically $x$ is not used as a realistic surrogate until a threshold is applied (for example, the deviation from lane center may be continuously monitored; $x$ may be used as a crash surrogate when it exceeds a defined threshold). In general, multiple variables may be combined as shown in Equation 4.2:

$X= \begin{cases}1 & \text { if } x_{1}>a_{1}, x_{2}>a_{2}, \ldots, x_{p}>a_{p} \\ 0 & \text { otherwise }\end{cases}$

(Here all $x_{i}>a_{i}$ are required to hold, but more complex logical relationships are also possible-for example, by using the OR operator.) It is clear that a very large number of candidate surrogates may be generated in this way.

According to the research hypotheses, it is intended to capture aspects of crash mechanisms in the surrogate, in the form of disturbed vehicle control by the driver. For road departure crashes this clearly relates to lateral (steering) control, so variables such as lane deviation and steering correction come to mind. Also, driver assistance systems - on suitably equipped vehicles-are designed to give alerts when an apparently high-risk scenario occurs; in the NDD (Chapter 3), such an alert was available as a candidate surrogate. Table 4.1 provides a list of basic candidate surrogates formulated, or at least initially considered, in the research project.

Table 4.1 is not a complete list. Indeed, given the opportunity to combine basic variables via algebraic and logical functions, the full list is actually infinite. Even when this table was used, it was not considered feasible to analyze all listed surrogates in depth, because of constraints on time as well as feasibility in some cases. The list is briefly reviewed below to explain the main aspects.

The first two aspects, lane departure warning (LDW) and curve speed warning (CSW) events, were recorded from the equipped vehicles used in the naturalistic driving study. Onboard systems used a lane-tracking camera, side-pointing radar, and a high-resolution digital road map, as well as vehicle variables such as turn signal, speed and steering angle, to compute (1) a warning that the vehicle is about to leave the lane when apparently the driver does not intend to do so and (2) a warning that the driver is approaching a curve too fast. While the full details of the algorithms are not published, it can be stated that most relevant aspects of these warning events can be reconstructed in postprocessing (motivating virtual lane departure warning [VLDW]). A virtual curve speed warning is not included in the list but is also feasible. However, it is worth noting that LDW makes use of the side-pointing radar to adapt the warning threshold. An estimate of "available maneuvering room" is made, so the event threshold is reduced when a crash barrier or an adjacent vehicle in detected by the radar, making a warning more likely. This aspect is not so easily incorporated into a post-hoc VLDW surrogate.

The next candidate, lateral deviation (LDEV), simply measures the instantaneous deviation from the lane center. This 
Table 4.1. Summary of Candidate Surrogates

\begin{tabular}{|c|c|c|c|}
\hline Name & Abbreviation & Type & Brief Description \\
\hline Lane departure warning & LDW & Boolean & Warning from lane departure warning system (RDCW project) \\
\hline Curve speed warning & CSW & Boolean & Warning from curve speed warning system (RDCW project) \\
\hline Virtual lane departure warning & VLDW & Boolean & $\begin{array}{l}\text { Kinematic condition based on postprocessing, aimed to emulate an onboard } \\
\text { LDW system }\end{array}$ \\
\hline Lateral deviation & LDEV & Continuous & Lateral deviation of vehicle from the lane center \\
\hline Predictive lateral deviation & PLDEV & Continuous & Predicted LDEV based on current motion and lane geometry \\
\hline Time to lane crossing & TTLC & Continuous & $\begin{array}{l}\text { Estimated time for the vehicle to leave travel lane, given the current speed, } \\
\text { position, and direction of motion }\end{array}$ \\
\hline Inverse time to lane crossing & ITTLC & Continuous & Reciprocal of TTLC \\
\hline Time to edge crossing & TTEC & Continuous & As TTLC, but including shoulder width \\
\hline Inverse time to edge crossing & ITTEC & Continuous & Reciprocal of TTEC \\
\hline Yaw rate error & YRE & Continuous & $\begin{array}{l}\text { Correction required to current yaw rate to avoid a deviation from the lane (with } \\
\text { a given time horizon) }\end{array}$ \\
\hline Control loop coherency & CLC & Continuous & $\begin{array}{l}\text { Signal processing-based measure of coherency between steer angle and LDEV } \\
\text { variable }\end{array}$ \\
\hline Visual interruption & VINT & Boolean & Driver looks away from the road for more than a specified time \\
\hline Steering rate & QSR & Boolean & Steering rate below a prescribed threshold \\
\hline $\begin{array}{l}\text { Yaw deviation associated with } \\
\text { a boundary discontinuity }\end{array}$ & YD-BD & Boolean & $\begin{array}{l}\text { Association of lane or road boundary discontinuity (e.g., missing lane marker) } \\
\text { with a lateral or yaw deviation }\end{array}$ \\
\hline
\end{tabular}

deviation is based on estimates from the vision-based lane tracker on the FOT vehicle, measuring the offset of the vehicle from the center of the lane. Applying a threshold to this (see the next section for more detail) may indicate some aspect of disturbed lateral control, but this ignores the way that drivers may knowingly or deliberately cut across lane boundaries. Indeed, this consideration was included in the LDW event algorithm, offering more lateral freedom when no "hard" objects were detected by the side radar. An improved measure of lane-keeping error is offered by predictive lateral deviation (PLDEV), where the vehicle path is extrapolated according to a horizon time (e.g., 1 or $2 \mathrm{~s}$ ) so that even if the vehicle is outside the lane boundary, the intent is to control or reduce the excursion. This presumably differs from an unintended drift out of lane (or drift toward the pavement edge), so an improved measure of control disturbance may result.

Time to lane crossing (TTLC) is somewhat similar to PLDEV. Given the current trajectory of the vehicle and assuming no change of speed or steer angle (and hence path curvature), TTLC is the estimated time for the relevant front wheel to cross the lane boundary. As a potential risk measure, its reciprocal-inverse time to lane crossing (ITTLC) - is perhaps preferred; in this case, a large value indicates proximity to lane departure, so "big is bad." On the other hand, when thresholds are used to define surrogate events, it is clear that the two variables are completely equivalent. Also, if the departure is referenced on the appropriate road boundary-so typically the shoulder width is included in the calculation-the corresponding measures are time to edge crossing (TTEC) and inverse time to edge crossing (ITTEC).

The above measures are based on relatively simple vehicle kinematics relative to the lane or road edges. In an attempt to overcome some of these limitations, YRE is defined as a measure of steering correction required to remain within the lane boundaries. The difference may appear minimal, but as will be developed later, this measure appears to offer numerous important advantages, the most important of which is that it infers a physical reference for an actively engaged driver to follow. The result is a criterion that is likely to be more directly related to the control task for lane keeping, something that is taken up in more detail in Chapter 7. In a related finding, if control input (steering) and reference (e.g., YRE or LDEV) are estimated, the quality of driving control performance may be inferred by a statistical relationship between the two. Because this relationship may not be instantaneous (e.g., the steering may exhibit a delayed or predictive response to a path or yaw error), a dynamic relationship is better considered, as, for example, in the form of a frequency-based measure of coherency, as broadly described in the control loop coherency (CLC) surrogate. 
The final three surrogates in Table 4.1 are more relevant to using combinations of candidate variables. Visual interruption (VINT) assumes that direct eye tracking (or at least head pose) of the driver is measured. In this case, when VINT is coincident with some other variable such as YRE, a surrogate event of "poor directional control while distracted" is motivated. Similarly, if the steering rate drops to near zero at the same time an YRE threshold is exceeded, the implication is that the driver is not responding appropriately to a yaw deviation. In a similar fashion, yaw deviation associated with a boundary discontinuity (YD-BD) indicates a yaw deviation associated with a break in the lane or road boundary. Presuming an increased probability of a missing or ambiguous reference for the driver causing the yaw excursion, this deviation provides another (bivariate) surrogate measure.

\section{Specific Surrogates Used for Analysis}

As mentioned earlier, it has not been possible to fully analyze all the above surrogates within the scope of this research. In the case of driver eye tracking or head pose, the required information is not directly available in the NDD. This study focused on a subset of these surrogates, and in the next sections the research team considers various aspects of LDW, LDEV, TTLC, TTEC, YD-BD, and YRE. In particular, the statistical analysis of LDEV, LDW, and TTEC events is presented in Chapter 5, so further details are now presented regarding how these events were defined.

\section{LDEV}

The vehicle offset was obtained at a rate of $10 \mathrm{~Hz}$ when the subject vehicle was in a lane with a solid right or left boundary, and lane-tracking confidence was $70 \%$ or higher. Periods of time when the turn signal was on were excluded, and only time intervals when the lateral velocity was in the direction of a solid lane boundary were used. The vehicle offset was calculated for the above conditions for both the right and left boundaries for the entire driving data set, and the 95th global percentile value of LDEV was obtained to be used as a threshold for identifying LDEV events. An LDEV event was defined as the incident when a vehicle exceeded the 95th percentile lane offset. At that time, to avoid multiple repetitions during the same event, the comparison (of offset against the 95th percentile) was suppressed for $10 \mathrm{~s}$ in the drive record, and then resumed. For example, if a vehicle offset greater than the 95th percentile was detected, the LDEV count increased by 1 . The next comparison of vehicle offset would occur $10 \mathrm{~s}$ later in the vehicle's time history.

\section{LDW}

In the FOT study that generated the NDD, the LDW was triggered when the predicted vehicle path was to cross a solid lane boundary (edge or centerline), or dashed line boundary into occupied space. The vehicle had to be traveling on a road that was not a local street, the vehicle speed had to exceed $25 \mathrm{mph}$, there was no turn signal or braking in the past $5 \mathrm{~s}$, and there was actual tracking on the boundary to be crossed. There was also a restriction on high steering rate in the past $5 \mathrm{~s}$. The circumstances of each LDW event in the naturalistic driving database were processed, so that only LDW events of solid right and left boundaries were retained. On the basis of the full data set, the average duration of the LDEV events was $0.61 \mathrm{~s}$ and the maximum was 8.9 s. A 10-s delay prevented the long events from artificially increasing the event count and excluded only $2.3 \%$ of the NDD.

\section{TTEC}

TTEC was extracted on the basis of position and velocity information. Unlike YRE (Chapter 7), TTEC did not directly include curvature of the road or the vehicle path. It was calculated as the quotient of the distance to the outside edge of the roadway (i.e., outer edge of shoulder) divided by the lateral vehicle velocity, as shown in Equation 4.3.

TTEC $=\frac{(d+\text { AMR })}{\mathrm{V}_{\text {lateral }}}$

This measure takes advantage of a variable in the NDD, called the available lateral maneuvering room (AMR), which was derived from side-radar reflection, and lane-tracking information (Figure 4.1).

The distance to the road edge was determined from the vehicle's position in the lane (from the vehicle offset from the center

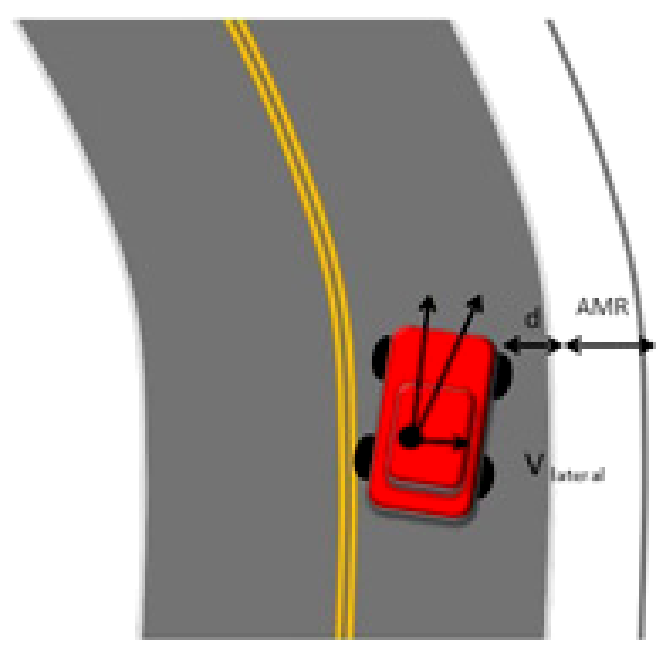

Figure 4.1. Illustration of TTEC. 
of the lane following the same method as used for LDEV), the width of the vehicle, and the available maneuvering room. Only periods of driving were considered when the lateral velocity points toward the right solid lane boundary with lateral velocity to the right. To be included, a driving period had to have tracking confidence $70 \%$ or better, and no turn signal. TTEC values were obtained for a rate of $10 \mathrm{~Hz}$ for all driving periods in the database that satisfied the above conditions. TTEC values in this form were used in the extreme value analysis.
The 5th percentile global value of TTEC was also determined and used as a threshold to define TTEC events for statistical modeling. A TTEC event was defined as an instance when the vehicle's TTEC was less than the 5th percentile global TTEC value. At that time, the comparison of TTEC against the 5th percentile value was suppressed for $10 \mathrm{~s}$ to prevent the long events from inflating the surrogate counts. This procedure generated the number of TTEC events for each directional segment in the analysis database. 


\section{Statistical Analysis: A Unified Approach to the Analysis of Rates for Crashes and Crash Surrogates}

\section{Traditional Analysis of Crash Data}

In transportation-related safety studies, various data analytic methodologies have been used to investigate associations between crashes and various risk factors. Historically, depending on the application under investigation, Poisson, negative binomial, random effects, and hierarchical Bayesian data models, among others, have been used to analyze data collected from historical crash databases. The response variable under these models is typically the number of crashes which can be cross-classified into a contingency table according to certain explanatory factors hypothesized to be associated with the response variable (Table 5.1). Exposure data, such as vehicle miles traveled (VMT) or annual average daily traffic (AADT), can also be cross-classified by the explanatory factors for the analysis of rates. As shown in Table 5.1, explanatory factors can include those related to the driver, the environment, the vehicle, and the highway. After an appropriate model is fitted to the data, relative risks (RRs) of various combinations of the explanatory variables, including interactions, can be calculated from the estimated model parameters to determine which risk factors are most associated with the occurrence of crashes.

This project involves an additional component not generally considered in standard data analysis problems. The study focuses on the statistical relationship between surrogate measures of collisions with actual collisions, and the formulation of exposure-based risk measures using these surrogate measures. The surrogate measures of collisions are generally collected from naturalistic driving data (NDD), which rarely provide sufficient data resulting from actual collisions, while data for crash outcomes are derived from historical crash databases. Therefore, it is necessary to consider the attributes of crash data and naturalistic driving data simultaneously in order to provide a link between crashes and crash surrogates (Table 5.2).
The variables collected from NDD are generally richer than those available in historical crash databases in the sense that NDD are derived from instrumented vehicles capable of making precise measurements with respect to certain roadway factors, driver behavior, and vehicle factors. Crash data, on the other hand, are derived mainly from the information available in police accident reports and cannot capture the level of detail contained in NDD. While certain variables such as weather condition, light condition, and road condition are recorded in both data sources, many variables recorded in natural driving experiments are not recorded in crash databases. Therefore, it is desirable to develop data analytic methods that consider crash data and NDD in a unifying framework, one that can account for and possibly adjust for inherent differences in the types of variables available from the two different sources of data.

In the analysis that follows, the response variables are actually defined as rates (crashes or surrogate event per unit exposure). Thus, exposure is included in the definition of the response variable in each case. However, exposure is also included in the explanatory variable set, so it is simply a matter of adjusting the model coefficients to convert from rates to counts and vice versa. In general, the relationship between crash (or surrogate) counts and exposure is expected to be nonlinear. However, on a logarithmic scale it is commonly the case that this relationship does indeed become linear (indicating a power law relationship in the underlying variables). In Figures 5.1 and 5.2, a plausible linear relationship is indeed seen on logarithmic scales.

\section{Seemingly Unrelated Regression Model}

Based on the discussion above, a model is proposed that extends the usual univariate response model for crashes to a model that treats crashes and crash surrogates as a bivariate response variable. Instead of fitting one model for crashes 
Table 5.1. Common

Response and Explanatory

Factors Used in Crash

Data Analysis

\begin{tabular}{|l|l|}
\hline \multirow{2}{*}{ Response } & Crashes \\
\cline { 2 - 2 } & (Exposure) \\
\hline \multirow{2}{*}{ Explanatory Factor } & Driver \\
\cline { 2 - 2 } & Environment \\
\cline { 2 - 2 } & Vehicle \\
\cline { 2 - 2 } & Highway \\
\hline
\end{tabular}

and independently fitting a separate model for a crash surrogate, the idea is to fit one model that accommodates both responses in a unifying model. The model is based on the method of seemingly unrelated regressions (SURs) proposed by Zellner (1962). SUR is developed in a normal theory framework and incorporates a correlation structure between crashes and crash surrogates. It allows formal tests of hypotheses to be conducted to test whether the risks associated with explanatory factors, or more importantly subsets of explanatory factors, are the same or different for crashes and crash surrogates.

The model used here takes the form shown in Equation 5.1:

$Y_{1}=X_{1} \beta_{1}+\varepsilon_{1}$

$Y_{2}=X_{2} \beta_{2}+\varepsilon_{2}$

where the subscript 1 refers to the crash model and the subscript 2 refers to the surrogate model. The equations resemble ordinary regression equations where $Y_{1}$ and $Y_{2}$ are the response variables, $X_{1}$ and $X_{2}$ are data matrices of explanatory variables, $\beta_{1}$ and $\beta_{2}$ are regression parameters, and $\varepsilon_{1}$ and $\varepsilon_{2}$ are error terms with normal distributions. In the SUR framework, the crash data are stacked on top of the surrogate data to form a system of equations (Equation 5.2).

Table 5.2. Simultaneous Consideration of Crash and Field Operational Test Data

\begin{tabular}{|l|l|l|l|}
\hline \multicolumn{2}{|l|}{ Crash Data } & \multicolumn{2}{|c|}{$\begin{array}{r}\text { Field Operational } \\
\text { Test Data }\end{array}$} \\
\hline \multirow{2}{*}{ Response } & Crashes & \multirow{2}{*}{ Response } & Surrogates \\
\cline { 2 - 2 } & (Exposure) & & $($ Exposure) \\
\hline \multirow{2}{*}{$\begin{array}{c}\text { Explanatory } \\
\text { Factor }\end{array}$} & Driver & \multirow{2}{*}{$\begin{array}{c}\text { Explanatory } \\
\text { Factor }\end{array}$} & Driver \\
\cline { 2 - 2 } & Environment & & Environment \\
\cline { 2 - 2 } & Vehicle & & Vehicle \\
\cline { 2 - 2 } & Highway & & Highway \\
\hline
\end{tabular}

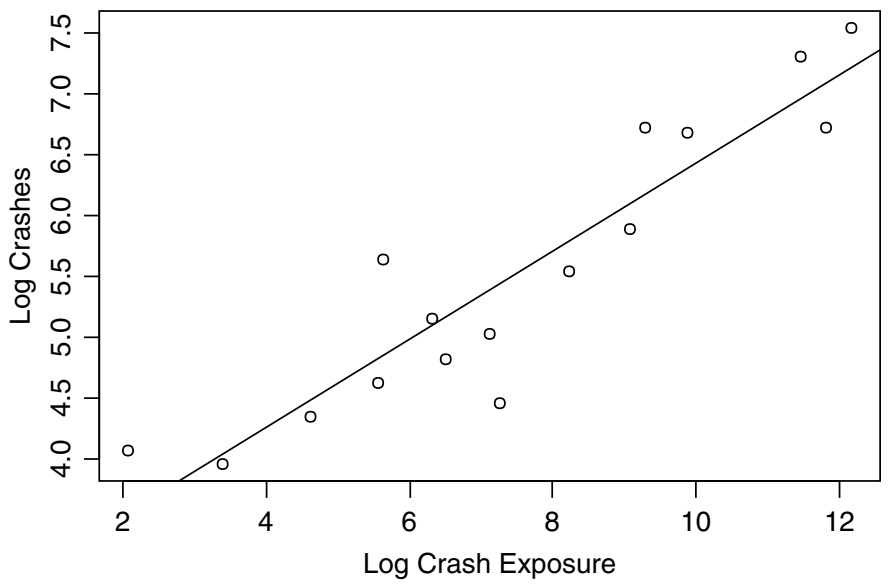

Figure 5.1. Relationship between crash numbers and exposure on logarithmic scales.

$\left[\begin{array}{l}Y_{1} \\ Y_{2}\end{array}\right]=\left[\begin{array}{ll}X_{1} & 0 \\ 0 & X_{2}\end{array}\right] \cdot\left[\begin{array}{l}\beta_{1} \\ \beta_{2}\end{array}\right]+\left[\begin{array}{l}\varepsilon_{1} \\ \varepsilon_{2}\end{array}\right]$

Note that the $X$ matrices of explanatory variables are not required to be the same, either in terms of variables or in terms of dimension. Therefore, variables collected from NDD can be different from those collected in the crash data set. Since crash data are stacked on top of surrogate data, the system of equations satisfies a linear model of the form shown in Equation 5.3:

$Y=X \beta+\varepsilon, \quad \varepsilon \sim N(0, \Sigma)$

where

$\operatorname{Var}(\varepsilon)=\Sigma=\left[\begin{array}{ll}\sigma_{11} I & \sigma_{12} I \\ \sigma_{21} I & \sigma_{22} I\end{array}\right]$

and $I$ is an identity matrix. Suppose $Y_{1}$ has dimension $N_{1} \times 1$ and $Y_{2}$ has dimension $N_{2} \times 1$ so that $Y$ has length $N_{1}+N_{2}=N$.

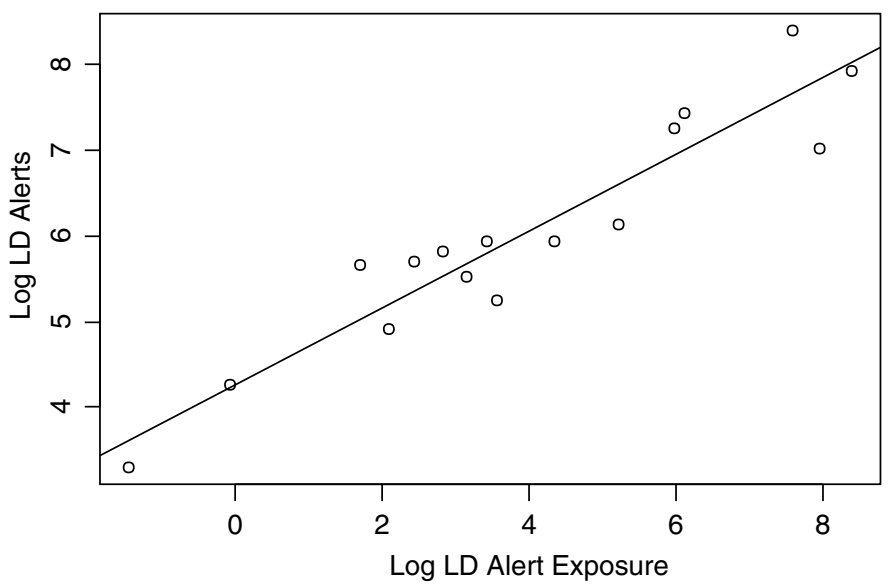

Figure 5.2. Relationship between surrogate crash numbers and exposure on logarithmic scales. 
Then, the matrix $\Sigma$ has dimension $N \times N$. Since this model satisfies the properties of a linear model with a defined covariance matrix, the parameters can be estimated by weighted least squares (WLS) as shown in Equation 5.4:

$\hat{\beta}=\left(X^{T} \Sigma^{-1} X\right)^{-1} X^{T} \Sigma^{-1} Y$

The parameters $\sigma_{11}$ and $\sigma_{22}$ represent the variances in the crash and surrogate regressions, respectively. The parameter $\sigma_{12}=\sigma_{21}$ is the covariance between $Y_{1}$ and $Y_{2}$. These parameters are estimated by fitting separate independent regressions for the crash data and the surrogate data and using the usual residual sum of squares for the variances, and the sum of the residual cross-product terms for the covariance.

The utility of this unifying framework is that tests of hypotheses of the form

$H_{0}: \beta_{1}=\beta_{2}$

can be conducted by using the usual $F$-test in a regression setting. This hypothesis tests whether crash model parameters equal surrogate model parameters. More important, it is possible to test whether only certain crash model parameters equal certain surrogate model parameters. This last point is important to the application of this framework to the simultaneous modeling of crashes and surrogates, because in many cases only a subset of the variables will be common to both.

\section{Poisson Log-Linear Models Estimated by Weighted Least Squares}

The Poisson log-linear model is the standard model for the analysis of rates. However, this model has limited use in practice because for a Poisson random variable, the mean is restricted to equal the variance. This has caused researchers to consider more flexible models such as negative binomial, generalized linear mixed models (GLMMs), or Bayesian models.

It is well known that WLS can be used to estimate maximum likelihood parameters in Poisson log-linear models (see, e.g., Agresti 2002). Therefore, the SUR framework can be used to estimate parameters in a log-linear model since parameters in a SUR model can be estimated by WLS. The WLS solution depends on asymptotic theory, so the only restriction is that the data are not too sparse. For example, the number of crashes or the number of surrogate events should not be 0 for many observations. Estimating parameters in a log-linear model by using normal theory on which the SUR model is based requires a square root transformation of the data. In particular, as shown in Equations 5.5 and 5.6, the dependent and independent variables are

$$
Y^{\prime}=\sqrt{Y} \log (Y) \quad X^{\prime}=\sqrt{Y} X
$$

The variable $Y^{\prime}$ is regressed on $X^{\prime}$ by using WLS with covariance matrix $\Sigma$. This model does not suffer from the restrictions of the Poisson model. That is, in addition to the mean parameter, the normal model has two parameters for variance, and one parameter for the covariance. Therefore, it can handle extravariability or overdispersion often encountered in observational studies that the standard Poisson model cannot. One disadvantage, discussed briefly above, is that $Y$ should not be 0 . In the rare cases that it is $0,0.5$ can be added. The asymptotics can break down if there are many zeros. Ideally, the $Y$ values should be at least 5 .

\section{Bayesian SUR for Log-Linear Models}

Now that the model is set up in the context of a normal theory linear model, the extension to a Bayesian model is straightforward. Methods for Bayesian data analysis of normal regression models are well developed. A likelihood function for the data and a prior distribution for the parameters must be specified. The likelihood function and prior distributions are described below in Equations 5.7 through 5.9.

Likelihood: $\quad Y^{\prime} \mid \mu, \Sigma \sim N(\mu, \Sigma) \quad \Sigma$ fixed

Prior 1:

$$
\begin{aligned}
\mu_{i} \mid \beta, \tau \sim N\left(\lambda_{i}, \tau\right) \quad i=1, \ldots, N \\
\lambda_{i}=\beta_{0} x_{i 0}^{\prime}+\beta_{1} x_{i 1}^{\prime}+\cdots \cdots+\beta_{p} x_{i p}^{\prime}
\end{aligned}
$$

Prior 2:

$$
\begin{aligned}
& \beta_{j} \sim N\left(0,10^{6}\right) j=0, \ldots, p \\
& 1 / \tau \sim \operatorname{Gamma}(0.001,0.001)
\end{aligned}
$$

In the likelihood, the matrix $\Sigma$ is assumed to be fixed. It is the same as described above and contains two parameters for variance, $\sigma_{11}$ and $\sigma_{22}$, and one parameter $\sigma_{12}$ for covariance. These parameters are estimated by using the residual sum of squares and residual sum of cross-product terms from ordinary independent regression models fit to crash and surrogate data, respectively. The regression model equation is incorporated into the first prior as the mean of a normal distribution and is designated by $\lambda$, which is a linear combination of the regression parameters $\beta$ and the explanatory variables $X$. The second prior is proper and takes a standard noninformative prior. Using proper priors ensures propriety of posterior distributions.

Estimation proceeds by Markov chain Monte Carlo (MCMC) simulation, which is used to generate random variables from the posterior distributions of the parameters $\mu, \beta$, and $\tau$. Because calculation of posterior distributions directly is not possible in closed form, the output generated from MCMC simulation is used to estimate characteristics of posterior distributions. These Markov chains are designed to converge in 
distribution to the desired posterior distributions. To ensure convergence, Markov chains are run with 60,000 iterations, and the first 30,000 are discarded for "burn-in."

The Bayesian model has an important advantage over the classical model. Because the regression model is specified in the prior, the posterior estimate of $\mu$ will tend to be a weighted average of the data $Y^{\prime}$ and the regression estimate $\lambda$. The weights depend on the estimates of variance, namely $\Sigma$ and $\tau$. Therefore, if the regression model displays lack of fit, indicated by large $\tau$, the posterior estimate will be smoothed toward the data. Accordingly, in the Bayesian SUR model, interest focuses on the posterior estimates of $\mu$ and not on the regression estimates $\lambda$. The estimates of RR produced by the Bayesian model that are the focus of this analysis depend on $\mu$. Since the Bayesian model produces estimates that are a weighted average of the data and the regression model, in the case of lack of fit the Bayesian model smoothes estimates toward the data. This was an important property in the models fit by the team. In a classical model, RR would be estimated by the regression equation for $\lambda$ alone.

Because the SUR model is estimated on a transformed scale to normality, it is necessary to transform back to make inference about the RRs. The RR is simply a ratio of rates comparing one combination of explanatory variables in the numerator to another combination in the denominator. Running the Markov chain will produce samples generated from the posterior distributions of $\mu$. The transformation of the dependent variable that was shown in the previous section is:

$Y^{\prime}=\sqrt{Y} \log (Y)$

Therefore, the simulated values should be transformed by the formula

$$
\frac{\mu}{\sqrt{Y}}-\log (\text { exposure })
$$

to calculate a posterior sample for the log rates. Then log RRs can be formed by taking differences of log rates based on combinations of certain explanatory variables. The reason for using the $\log R R$ is that the sampling distribution of the RR on the log scale is close to normal. A main hypothesis of interest is whether the difference between a crash $\log R R$ and a surrogate $\log R R$ is 0 while controlling for certain explanatory variables.

\section{SUR Model Application and Results}

Bayesian SUR models were applied to right road departure crashes and three candidate surrogates: right lane deviation (LDEV), right lane departure warning (LDW), and time to right edge crossing (TTEC). The number of explanatory variables for SUR model application was limited by the data and consisted of four variables reported in the literature to be associated with road departure crashes, area type, road type, horizontal curvature, and shoulder width. Therefore, three separate models are presented, one for each crash-surrogate pair. The categorical models are: crashes, surrogate events, and exposure measures aggregated into the 24 combinations of the four variables in the models-Curve (2), Freeway (2), Area (2), and Right Shoulder (3) - so that there are $2 \times 2 \times 2 \times 3=24$ independent observations. Of the 24 possible cells (combinations of the explanatory variables), only 16 are used as data for the models; six cells were necessarily empty (meaning the specific combinations were not found in the data, for example, rural freeways with shoulder width 0 to $3 \mathrm{ft}$ on curved and on tangent sections), and two cells had very low values for traversals and crashes, that dropped from the analysis. The number of traversals for the cells left in the analysis ranged from 57 to more than 28,000 , and the number of crashes in the cells ranged from 52 to 1,879 .

The exposure for crashes in each case was based on the 5-year traffic volume and segment length, and the exposure for each of the surrogates was based on the number of traversals in segment and segment length. The same set of explanatory variables was used in each model:

- Curve $(1=$ Yes, 2 = No $)$

- Freeway $(1=$ Yes, 2 = No)

- Area (1 = Rural, 2 = Urban $)$

- Right Shoulder $(1=0$ to $3 \mathrm{ft}, 2=3+\mathrm{ft}$ to $8 \mathrm{ft}, 3=8+\mathrm{ft}$ )

The data used in each of the models for each of the three surrogate candidates are in Appendix C. Results from the three are shown in subsequent subsections. Posterior estimates of the regression parameters are given, and log RR comparisons between crashes and surrogate measures are shown. The real focus of this analysis is the presentation of the log RR differences, which are used to determine if the RRs of crashes and surrogate events are the same under specified conditions. The regression parameters in the crash and surrogate equations are of secondary concern. The regression parameters in the models are shown to give an indication of the effects of the four variables on crashes and surrogate measures. Often, parameter estimates are in the same direction and are of similar magnitude in the crash and surrogate regression equations. The regression model was included in the prior specification of the Bayesian model to help smooth estimates of RR, the primary exposure-based risk measure used in the study.

In the regression equations, the first level of each independent variable serves as the baseline case in which parameter estimates are constrained to be 0 . That is, for the binary variables Curve, Freeway, and Area, the parameter estimates are associated with the second level of the categorical variables. For example, a negative regression coefficient attached to Curve indicates that a crash or surrogate event is more likely 
on a curve than on a segment of highway defined as not on a curve. The Right Shoulder variable has two regression parameters corresponding to the second level $(3+\mathrm{ft}$ to $8 \mathrm{ft})$ and third level $(8+\mathrm{ft})$ of that variable.

The $\log$ RR of a crash and of each candidate surrogate were calculated from the three models for a road segment with a curve compared to a road segment without a curve on a nonfreeway rural road with shoulders greater than $3 \mathrm{ft}$ but less than $8 \mathrm{ft}$. If the log RR of a crash and a candidate surrogate are the same, then it is argued that the candidate is a good surrogate for the crash. Accordingly, for each model a sample is generated from the posterior distribution of the log RR difference by using MCMC simulation. The hypothesis of interest is whether 0 is contained in the middle $95 \%$ of this distribution. Note that one expects crash rates to be considerably smaller than rates derived from surrogate measures. This difference makes the RR an attractive exposure-based measure, since rates are not compared on an absolute scale but on a relative scale that compares the risk of an event on a curve to the risk of an event not on a curve.

\section{Lateral Deviation}

Table 5.3 shows posterior estimates from the regression parameters in the LDEV model. The table also includes estimates that describe the middle $95 \%$ of the distributions which

Table 5.3. Posterior Regression Estimates for Bayesian SUR LDEV Model

\begin{tabular}{|l|r|r|r|r|}
\hline $\begin{array}{l}\text { Crash } \\
\text { Parameter }\end{array}$ & Mean & SD & $\mathbf{2 . 5 \%}$ & $\mathbf{9 7 . 5 \%}$ \\
\hline Intercept & 2.095 & 0.419 & 1.263 & 2.933 \\
\hline Log exposure & 0.469 & 0.042 & 0.386 & 0.554 \\
\hline Curve & -0.642 & 0.072 & -0.782 & -0.500 \\
\hline Freeway & 0.262 & 0.126 & 0.012 & 0.510 \\
\hline Area & -0.534 & 0.216 & -0.967 & -0.113 \\
\hline Shoulder2 & 0.523 & 0.129 & 0.267 & 0.778 \\
\hline Shoulder3 & 0.327 & 0.145 & 0.040 & 0.615 \\
\hline LDEV & Mean & SD & $2.5 \%$ & $97.5 \%$ \\
\hline Intercept & 3.981 & 0.203 & 3.569 & 4.374 \\
\hline Log exposure & 0.553 & 0.030 & 0.494 & 0.613 \\
\hline Curve & -0.558 & 0.062 & -0.680 & -0.433 \\
\hline Freeway & -0.153 & 0.079 & -0.306 & 0.005 \\
\hline Area & -0.568 & 0.141 & -0.849 & -0.290 \\
\hline Shoulder2 & 0.658 & 0.090 & 0.477 & 0.836 \\
\hline Shoulder3 & 0.794 & 0.103 & 0.594 & 1.006 \\
\hline
\end{tabular}

Note: $\mathrm{SD}=$ standard deviation. are indicated by the 2.5 and 97.5 percentiles. Log exposure is fit on the right-hand side of the model equations for both the crash and the surrogate regressions. There is some similarity in the directions and the magnitudes for certain variables. For example, in the crash regression, the posterior mean for the Curve variable is -0.642 , while in the LDEV regression the value is -0.558 . On the basis of coding of the Curve variable, this difference suggests that crashes and LDEV events were more likely on curves. In addition, the Area variables are both negative and of similar magnitude. This similarity suggests the protective effects of urban areas relative to rural areas. The shoulder variables are both positive, although of somewhat differing magnitudes between the two regressions. The freeway variable is not significant at the 0.05 level in the LDEV regression.

At first glance, the signs of the coefficients for the shoulder width seem counterintuitive: apparently crash risk is higher when the shoulders are wider. Care is needed in interpretation. This result does not imply that increasing shoulder width on a particular road segment would increase crash risk. Rather, it indicates that, within the resolution of the statistical model used here, there is a systematic effect that more road departure crashes (as well as more surrogate events) occur under conditions where shoulders are wider than where shoulders are narrower. Note that a single model has been used for both urban and rural areas, and only a limited set of highway variables has been included. In urban areas, with high traffic density, shorter journey distances and occasional congestion, single-vehicle road departure crashes are relatively rare; curbs typically define the road edges (shoulder width is zero) and risk is low. On rural highways with higher traffic speeds (and shoulders present) the risk is expected to be higher. It is not surprising that the road segments of higher risk for road departure crashes are also areas with shoulders present. And if wider shoulders tend to be associated with limited access highways, higher speeds and longer journey times, again the association with higher risk is not too surprising. The urban/ rural area variable is expected to account for some of this variation (crash risk being lower for urban areas) but if the effect noted is particularly strong and the population-based "area" variable in only partially correlated with road conditions, it is not surprising that the presence of shoulders is associated with higher crash risk. Clearly it would be fruitful to increase the number of explanatory variables so that the shoulder variable is not confounded with other factors, and in the future, with larger data sets, this is entirely feasible. It will also be beneficial to implement separate models for urban and rural areas. (In this project, given the limited volume of driving data, it was more feasible to combine the two areas in a single model.) Thus, on the basis of the largescale naturalistic driving study, the team expects confounding effects to be removed, and the shoulder-width coefficients will provide a more direct indicator of relative risk. 

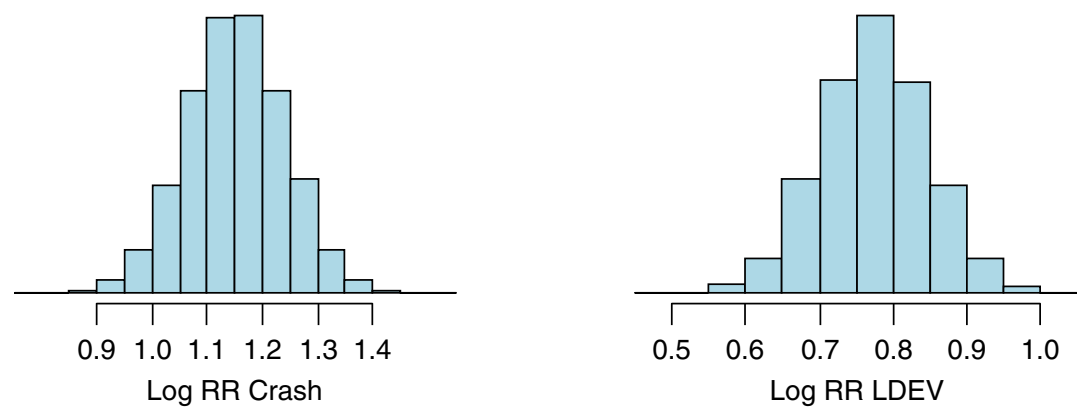

Figure 5.3. Posterior distributions of log RR comparing curve to no curve for crash and LDEV.

The real focus of this analysis, however, is not on the regression parameters, but the RR measures, which depend on the estimated posterior rates. One of the advantages of this Bayesian model is that the posterior rates tend to a weighted average of the observed rates and those estimated from the regression equation shown in Table 5.3. Therefore, if the regression equation displays lack of fit, the posterior estimates tend to the observed rates. Figure 5.3 shows histograms of samples of size 30,000 drawn from the posterior distributions of the $\log$ RRs for crashes and the LDEV surrogate. The comparison is between curved road segments and those that are not curved while holding the freeway, area, and shoulder variables fixed as described above in the section SUR Model Application and Results. The estimate for the log crash RR is 1.15 with a $95 \%$ confidence interval of $(0.98,1.33)$. The estimate for the log LDEV RR is 0.77 with a $95 \%$ confidence interval of $(0.63,0.92)$. Both of the confidence intervals do not contain 0 , suggesting that the risks of crashes and LDEV events are greater on curves holding the other variables fixed.

Figure 5.4 shows the posterior distribution of the $\log \mathrm{RR}$ difference between crashes and the LDEV surrogate comparing

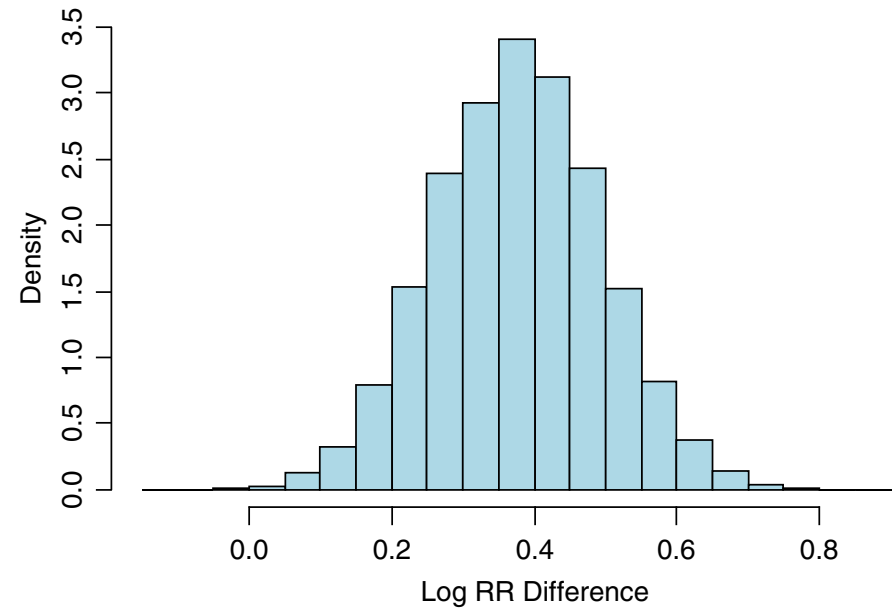

Figure 5.4. Posterior distribution of log RR difference between crash and LDEV comparing curve to no curve. curve to no curve while holding other variables in the model fixed. The mean of this distribution is 0.38 with a $95 \%$ confidence interval of $(0.15,0.61)$. Since 0 is not contained in the confidence interval, the conclusion is that lane deviation is a poor surrogate for lane departure crashes.

\section{Lane Departure Warning}

Table 5.4 shows posterior estimates from the regression parameters in the LDW model. In this model, the coefficients for the Curve variable are also negative, suggesting that crashes and

Table 5.4. Posterior Regression Estimates for Bayesian SUR LDW Model

\begin{tabular}{|l|c|c|c|c|}
\hline $\begin{array}{l}\text { Crash } \\
\text { Parameter }\end{array}$ & Mean & SD & $2.5 \%$ & $97.5 \%$ \\
\hline Intercept & 1.918 & 0.420 & 1.044 & 2.720 \\
\hline Log exposure & 0.463 & 0.039 & 0.391 & 0.546 \\
\hline Curve & -0.629 & 0.069 & -0.766 & -0.494 \\
\hline Freeway & 0.580 & 0.213 & 0.169 & 1.018 \\
\hline Area & -0.240 & 0.234 & -0.729 & 0.194 \\
\hline Shoulder2 & 0.486 & 0.119 & 0.258 & 0.728 \\
\hline Shoulder3 & 0.315 & 0.134 & 0.057 & 0.591 \\
\hline Freeway $\times$ area & -0.367 & 0.195 & -0.764 & 0.008 \\
\hline LDW & & & & -0.54 \\
\hline Parameter & Mean & SD & $2.5 \%$ & $97.5 \%$ \\
\hline Intercept & 1.536 & 0.654 & 0.285 & 2.833 \\
\hline Log exposure & 0.422 & 0.087 & 0.238 & 0.589 \\
\hline Curve & -0.522 & 0.174 & -0.864 & -0.184 \\
\hline Freeway & 0.866 & 0.498 & -0.071 & 1.885 \\
\hline Area & 0.428 & 0.580 & -0.709 & 1.565 \\
\hline Shoulder2 & 0.388 & 0.250 & -0.109 & 0.870 \\
\hline Shoulder3 & 0.643 & 0.293 & 0.062 & 1.212 \\
\hline Freeway $\times$ area & -0.964 & 0.485 & -1.919 & -0.045 \\
\hline
\end{tabular}



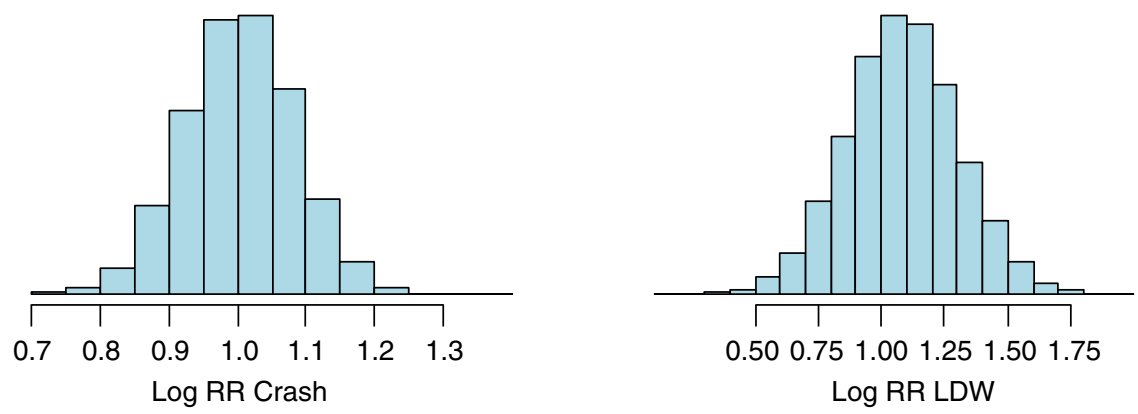

Figure 5.5. Posterior distributions of the log RR comparing curve to no curve for crash and LDW.

LDW events are more likely on curved road segments. The shoulder coefficients are also both positive. This model contains an interaction term between the freeway and area variables that is significant in the LDW regression equation and marginally significant in the crash regression. The negative coefficients suggest that the additive effects of freeway and area are somewhat reduced in urban areas when not on a freeway.

Figure 5.5 shows histograms of samples of size 30,000 drawn from the posterior distributions of the log RRs for crashes and the LDW surrogate. The estimate for the log crash RR is 1.00 with a $95 \%$ confidence interval of $(0.84,1.16)$. The estimate for the $\log$ LDW RR is 1.09 with a $95 \%$ confidence interval of $(0.65$, 1.53). Both of the confidence intervals do not contain 0 , suggesting that the risks of crashes and LDW events are greater on curves holding the other variables fixed.

Figure 5.6 shows the posterior distribution of the log RR difference between crashes and the LDW surrogate comparing curve to no curve while holding other variables in the model fixed. The mean of this distribution is -0.08 with a $95 \%$ confidence interval of $(-0.51,0.33)$. The $95 \%$ confidence interval for the log RR difference includes 0 , indicating that LDW could be useful as a surrogate for crashes on rural nonfreeway roads.

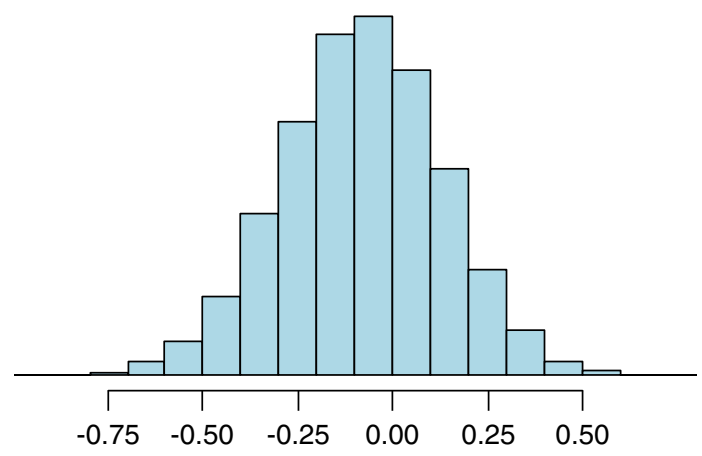

Figure 5.6. Posterior distribution of the $\log R R$ difference between crash and LDW comparing curve to no curve.

\section{Time to Edge Crossing}

Table 5.5 shows posterior estimates from the regression parameters in the TTEC model. In terms of regression estimates between the crash and surrogate measure, this model shows the best agreement among the three models. The log exposure, curve, area, and shoulder variables are not only in the same direction between the crash and the TTEC regressions, but the magnitudes also tend to be reasonably close. Note that the intercept is of no interest because it only captures the difference on an absolute scale of numbers of crashes and TTEC events. All parameters in the crash regression are significant. The freeway and area variables in the TTEC regression equation do not meet the significance criteria at 0.05 .

Table 5.5. Posterior Regression Estimates for Bayesian SUR TTEC Model

\begin{tabular}{|l|c|c|c|c|}
\hline $\begin{array}{l}\text { Crash } \\
\text { Parameter }\end{array}$ & Mean & SD & $\mathbf{2 . 5 \%}$ & $\mathbf{9 7 . 5 \%}$ \\
\hline Intercept & 2.017 & 0.438 & 1.136 & 2.836 \\
\hline Log exposure & 0.478 & 0.045 & 0.394 & 0.567 \\
\hline Curve & -0.638 & 0.077 & -0.787 & -0.488 \\
\hline Freeway & 0.285 & 0.130 & 0.038 & 0.545 \\
\hline Area & -0.579 & 0.230 & -1.033 & -0.129 \\
\hline Shoulder2 & 0.541 & 0.135 & 0.283 & 0.805 \\
\hline Shoulder3 & 0.351 & 0.152 & 0.063 & 0.653 \\
\hline $\begin{array}{l}\text { TTEC } \\
\text { Parameter }\end{array}$ & Mean & SD & $\mathbf{2 . 5 \%}$ & $\mathbf{9 7 . 5 \%}$ \\
\hline Intercept & 4.557 & 0.341 & 3.843 & 5.213 \\
\hline Log exposure & 0.464 & 0.054 & 0.362 & 0.576 \\
\hline Curve & -0.594 & 0.100 & -0.788 & -0.399 \\
\hline Freeway & 0.072 & 0.150 & -0.218 & 0.368 \\
\hline Area & -0.469 & 0.259 & -0.996 & 0.026 \\
\hline Shoulder2 & 0.462 & 0.150 & 0.172 & 0.765 \\
\hline Shoulder3 & 0.466 & 0.180 & 0.119 & 0.829 \\
\hline
\end{tabular}



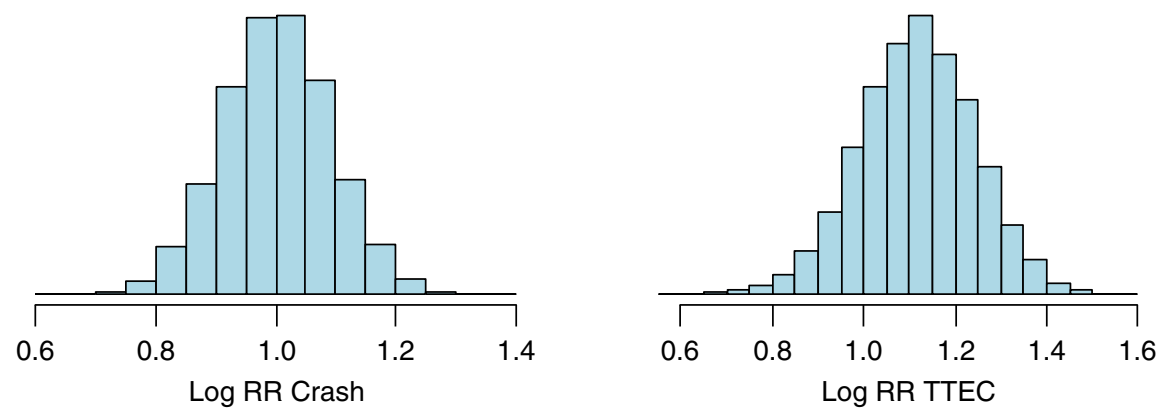

Figure 5.7. Posterior distributions of the log RR comparing curve to no curve for crash and TTEC.

Figure 5.7 shows histograms of samples of size 30,000 drawn from the posterior distributions of the log RRs for crashes and the TTEC surrogate. The estimate for the log crash RR is 1.00 with a $95 \%$ confidence interval of $(0.82,1.18)$. The estimate for the $\log$ TTEC RR is 1.12 with a $95 \%$ confidence interval of $(0.83,1.36)$. Both of the confidence intervals do not contain 0 , suggesting that the risks of crashes and TTEC events are greater on curves holding the other variables fixed.

Figure 5.8 shows the posterior distribution of the $\log \mathrm{RR}$ difference between crashes and the TTEC surrogate comparing curve to no curve while holding other variables in the model fixed. The mean of this distribution is -0.11 with a $95 \%$ confidence interval of $(-0.40,0.18)$. The $95 \%$ confidence interval for the $\log$ RR difference includes 0 , indicating that TTEC could be useful as a surrogate for crashes on rural nonfreeway roads.

Overall, from the three example analyses, it is seen that different candidate surrogates have different qualities in terms of their fidelity to the crash model. In the figures above, comparing log RR differences, as well as signs and magnitudes of the regression parameters, it is clear that LDEV is the worst candidate of the three, and TTEC is the best candidate, while LDW is intermediate. The results not only help confirm surrogacy but also provide a possible tool for guiding future studies in reducing risk: given a valid surrogate —one that really mimics RR in crashes_-an

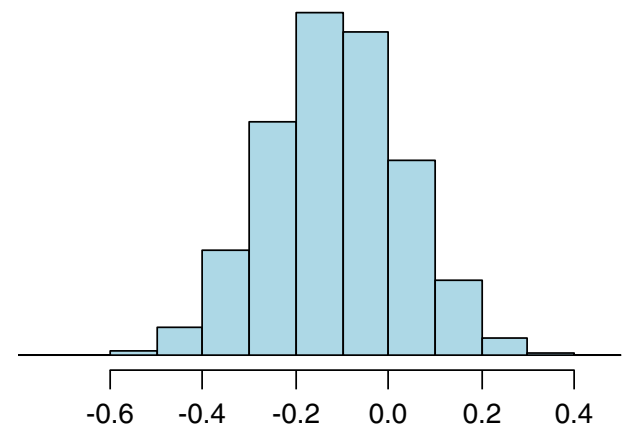

Figure 5.8. Posterior distribution of the log RR difference between crash and TTEC comparing curve to no curve. intervention such as road widening, improving lane markings, changing signage or adding rumble strips can be evaluated by using the surrogate in a relatively short space of time. The effect on RR then represents a predicted safety benefit. While data still need to be collected to evaluate the effect on the surrogate, this approach is potentially much more useful, sensitive, and repeatable than counting crashes at a single "treated" location.

This chapter explored a statistical approach for testing candidate surrogate measures for road departure crashes with the type of NDD and highway data that will be available from the SHRP 2 Safety projects. The focus was on identifying appropriate analysis methods coupled to mechanisms and control performance, not just on statistical associations. Three alternative surrogates were tested by using the SUR approach and using the same four explanatory variables - area type, road type, horizontal curvature, and shoulder width-that were used in the SUR analyses of the three candidate surrogates. These variables were selected a priori for this exploratory study because they are known to be associated with road departure crashes. Furthermore, classification of these variables was limited by available data. The results obtained were similar to what would be obtained from multivariate response model. The use of the same variables for both the crash and surrogate portions of the model was done for simplicity during the testing of the approach. The SUR framework developed in this section can accommodate the more general case, that is different variables can be used in the crash and surrogate portions of the model, bridging crash data with naturalistic data in a highway context. In particular, variables relating to specific driver behaviors are only available in the naturalistic case, so this flexibility in the SUR approach should prove valuable in the future. The large natural use data from SHRP 2 should provide a richer selection of explanatory variables and finer classification of explanatory variables for expanded analysis within this framework. The team's analysis has shown that the SUR approach is well suited for screening surrogates. Of the three surrogates evaluated, TTEC appears to be the best. However, the team's analysis was exploratory, and better surrogates may exist. 


\section{CHAPTER 6}

\section{Statistical Analysis: An Approach Using Extreme Value Theory}

\section{Extreme Value Analysis}

Another way to analyze crash surrogates is in terms of the underlying continuous measurements rather than from discrete surrogate events. This offers a possible way to estimate the probability of crash or near crash from the frequency of small crash margins. Extreme value theory provides a robust statistical method by which probability levels in the tails of observed distributions of crash margins can be estimated. One potential advantage is to link absolute exposure values (AADT) to actual crash numbers via surrogate estimated frequencies. Another advantage is that an objective surrogate threshold can be used in place of the more arbitrary percentile thresholds used previously.

Gumbel (1958) laid the foundation for the study of extreme values. Since then, extreme value theory has received much attention and undergone many changes. The idea is to model rare events that lie outside the range of available observations. The problem begins by selecting the largest (smallest) observation from each of many samples. The resulting sample of maximum (minimum) values is the sample of extreme values for analysis. Gumbel showed that for large samples, depending on the parent distribution, distributions of extremes can follow one of three asymptotic distributions. The three asymptotic distributions that Gumbel referred to as the first, second, and third asymptotes are now commonly called the Gumbel, Frechet, and Weibull distributions, respectively.

A generalized extreme value (GEV) distribution contains a parameter for accommodating all three solutions simultaneously so that a sample of extreme values can be fit to one distribution without consideration of the three cases separately (see, for example, Coles 2001). One approach is to make use of extremal probability paper based on the first asymptotic solution (the Gumbel distribution). If the observed data follow the Gumbel distribution, the data should plot as a straight line. If, however, the data follow the Frechet or Weibull distribution, the points will plot as a curve.
The distinguishing feature of an extreme value analysis is the objective to quantify the stochastic behavior of a process at unusually large (small) levels. Extreme value analyses usually require estimation of the probability of events that are more extreme than any that have already been observed. As an example, traffic crashes are generally regarded as rare events and few, if any, actual crashes may be observed during a field operational test using instrumented vehicles in a naturalistic driving study. Instead, surrogate measures may be defined that approach actual crashes if extrapolation is permitted from observed levels of the surrogate measures to unobserved levels.

The TTEC variable is used in this demonstration of the use of extreme value theory in the search for crash surrogates. With TTEC, the event of concern is road departure, a necessary but not sufficient condition for a road departure crash. However, extreme value analysis can help to explore relationships between road departures and road departure crashes by providing a surrogate for a surrogate, and thus leading to a better understanding of the road departure crash.

To demonstrate this approach it was necessary to identify a length of roadway traveled by a large number of subjects. An extreme value distribution can be fit to a sample of the minimum TTEC for each driver. By plotting the values on Gumbel probability paper, the rate at which vehicles run off that particular roadway can be estimated. Screening the analysis database for adjacent HPMS segments traversed by a large number of same subjects was not fruitful because the total number of drivers was not large, and their trips are distributed over a very large area. A search was conducted to find a single long road segment that was traversed by the largest number of drivers. The search yielded a 2.3-mi segment of US-23, a freeway in the Ann Arbor, Michigan, area which had 117 traversals by 43 different drivers. The segment has two lanes in each direction, a center median, a long horizontal reverse curve, and 12 - $\mathrm{ft}$ shoulders.

The minimum TTEC values for the 117 trips were used to fit the extreme value distribution. Treating traversals as 


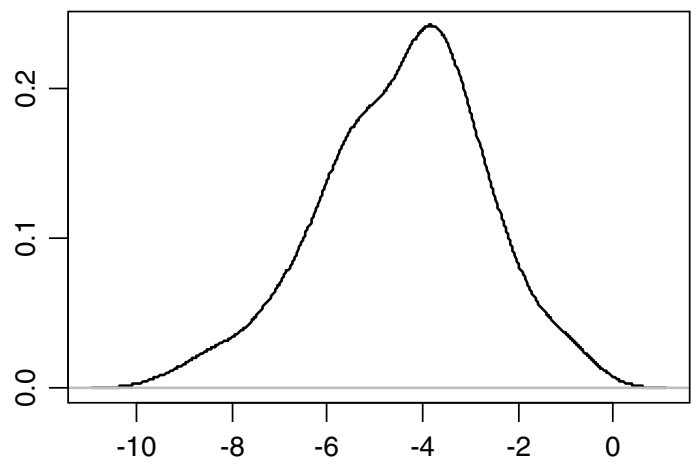

Figure 6.1. Kernel density plot of TTEC.

observations may violate an assumption of independence but results in larger sample size. It is possible that the normal differences in the environmental and traffic conditions encountered by the same driver on this road segment would justify treating each traversal as independent. An attempt was made to find differences due to night and rain conditions, but the numbers of traversals at night and during rain were too few for meaningful analysis.

Figure 6.1 shows a kernel density estimate of the minimum TTEC for the 117 observations. Standard extreme value analyses usually consider maximum values, but interest in TTEC is related to analysis of minimum values. One way to proceed with an extreme value analysis for minimum values is to reverse the sign of TTEC and simply conduct a standard extreme value analysis for maximum values. This was done, and hence the shown TTEC values are negative (the actual distribution is the mirror image of the distribution shown, symmetric about zero). The smoothed plot covers the value 0 .
Figure 6.2 shows the fit of a GEV distribution to the minimum TTEC data. The data are plotted on extreme value probability paper. Because the data plot as a curve that is increasing at a decreasing rate, the data tend to follow a Weibull distribution.

The assumption is that a road departure event occurs when TTEC $=0$, so interest focuses on the intersection of the fitted line and the horizontal line where TTEC $=0$. For these data, no observations resulted in actual events. Solution of the point where TTEC crosses 0 gives a return period of approximately 2 million. The return period is the reciprocal of the probability and can be used to estimate the expected number of observations required to attain a certain level of TTEC. For this example, one would expect to record approximately 2 million extreme observations before seeing one road departure.

The AADT for this road segment obtained from the HPMS data files is 65,755 vehicles. Thus, a road departure on this segment can be expected about once every 30.4 days or about 12 times a year. There were nine roadway departure crashes on this road segment from 2001 to 2005, or an average of 1.8 crashes per year. This indicates that on this particular road segment, about $15 \%$ of road departures resulted in a road departure crash. While the TTEC data are quite sparse, and these calculations cannot be seen as definitive, the resulting estimates appear very reasonable. This supports the potential future value in conducting an in-depth study based on extreme value theory and TTEC.

Figure 6.3 shows the distribution of crashes and TTEC extremes on the $2.3 \mathrm{mi}$ of US-23 near Ann Arbor. Here the research team has not attempted to correlate TTEC extremes with actual crash locations but noted that for this segment, the crashes are neither uniformly spaced nor clustered around

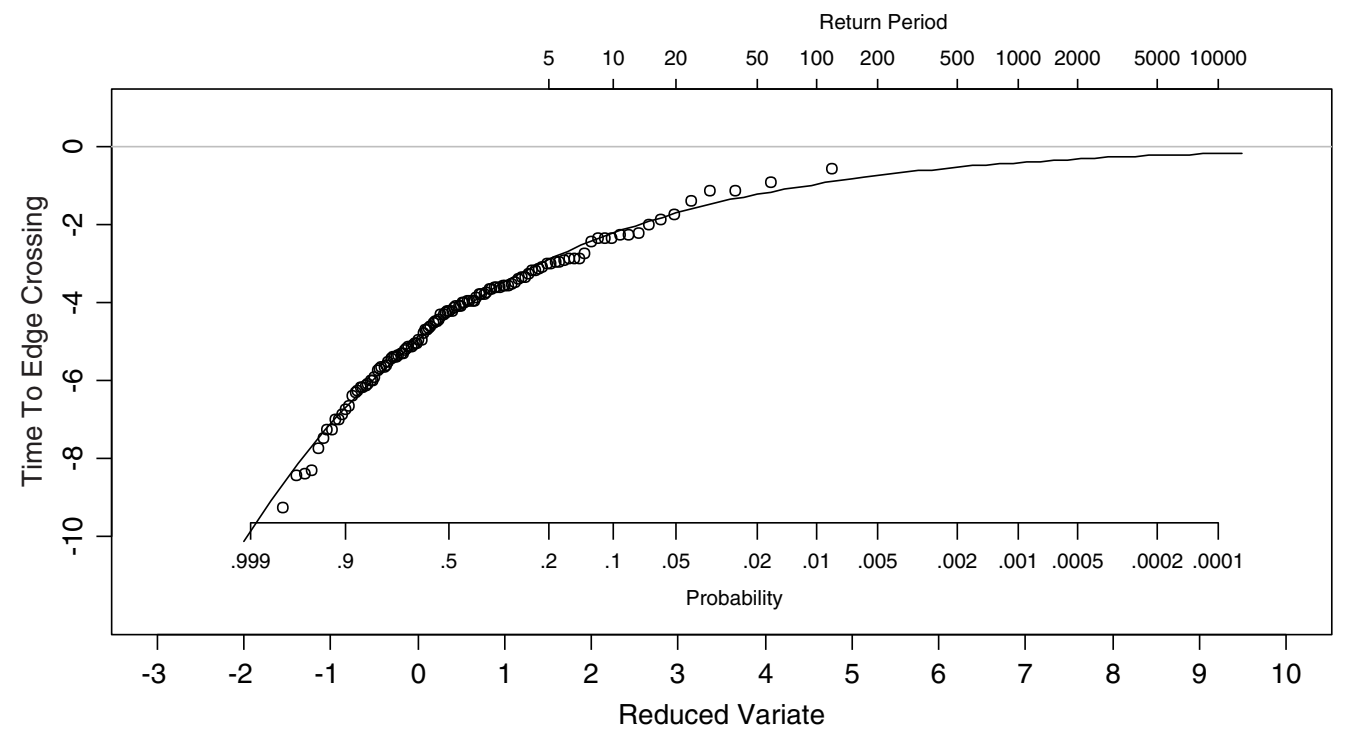

Figure 6.2. Fit of the generalized extreme value distribution to TTEC. 
$\triangle$ Crash

- TTEC

Extremes

Source: Google Earth.

Figure 6.3. Crashes and TTEC extremes on 2.3 miles of US-23.

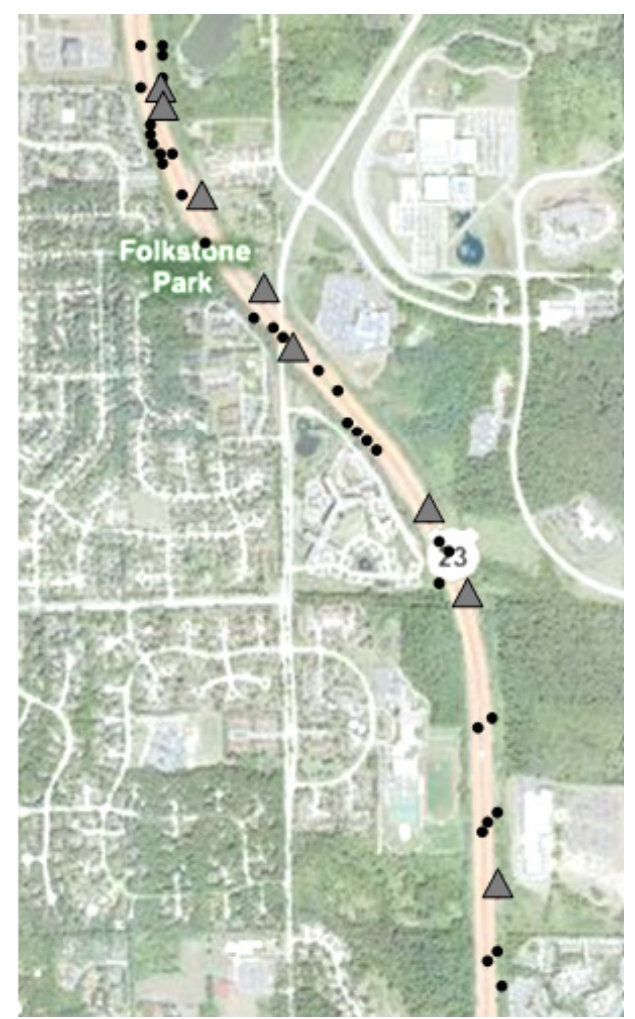

particular points. It therefore seems unlikely on the one hand that further analysis will establish meaningful trends of crash and surrogate event locations within individual segments. On the other hand, the relationship between frequencies of TTEC-based excursions (TTEC $\leq 0$ ) to actual crash numbers across different segments may be fruitful, because that relationship offers the possibility of deriving a nonparametric relationship between crashes and surrogate events. The TTEC $\leq 0$ surrogate event frequency, which is derived from extreme value theory, may be viewed as a higher-order surrogate event to be modeled via SUR in the same way other surrogates were considered in the previous section. As with several other aspects of the team's exploratory analysis, the possibilities are deferred to future studies based on the larger NDD to be collected under SHRP 2.

This section explored the use of extreme value analysis for examining properties of a surrogate. In contrast to the SUR approach that required categorical variables and used crash data, highway data, and NDD, the extreme value analysis presented here used only continuous natural use data. The linkage to crashes was done afterward by estimating the number of observations one would expect to record before seeing one road departure. The potential of the extreme value approach is in the further exploration of driver behaviors associated with small crash margin events in general or by specific roadway features. 


\section{OHAPTER Z Yaw Rate Error}

A key objective of this project has been to focus on data analysis coupled to mechanisms and control performance, not just on statistical associations. In Chapter 5 three candidate surrogates were evaluated, and time to edge crossing (TTEC) was seen to be the best, while the very simple lateral deviation variable provided the worst surrogate. Presumably TTEC works better because it takes account of more relevant variables. However, it may not be optimal and better surrogates may still exist. According to the guiding principles of Chapter 2, a best-case surrogate is expected to be closely connected to the control task and disturbances in the control task. In this section the aim is to formulate a candidate surrogate and evaluate its performance in a limited set of cases. However, because of time and computing constraints, at this point no formal largescale analysis using seemingly unrelated regression (SUR) or extreme value distributions is to be carried out.

When a driver is engaged in lane keeping, there exists a relatively continuous process of adjusting the yaw angle of the vehicle to match that of the road. On a continuous curve, the steer angle is changed so that the yaw angular velocitythe rate of change of directional angle-matches the highway curvature and the vehicle speed. Yaw rate error (YRE) is a proposed measure of overall lane-keeping control performance that may supersede simpler measures such as lane position or time to lane crossing discussed previously. While a full surrogate analysis based on YRE has not been carried out in this study, the team does focus on the feasibility of defining and computing YRE and seeks indicators of its value as a controloriented performance indicator. This brings the team back to "what driving is."

Driving is a control task based on visual input; it includes filtering of input for relevance, extracting signals or patterns from that visual information, and hence provides a reference to guide steering and speed control. Control action then involves manual effort by the driver to modulate vehicle motion using further force and acceleration cues (Toffin et al. 2007; Land and Horwood 1995; Chen and Ulsoy 2001).
Here the team's focus is on the visual reference for lane keeping in terms of a conflict measure or error criterion. In broad terms, the team seeks a simple measure of the control reference for situations when the driver is concerned with staying in the lane but less concerned with some optimal path within that lane. To this end, YRE, a measure of yaw rate correction required, is introduced. Since no preferred path is known, the YRE is computed for multiple lane boundary points and the most critical of these will represent the overall correction required. This metric has been used previously in driver modeling (Gordon and Magnuski 2006) and applied to collision avoidance (Chang and Gordon 2008).

The approach is analogous to longitudinal speed control in traffic, in which control action required can be found in terms of the vehicle deceleration needed to avoid a collision with the vehicle in front. Again, this contrasts with the predicted time to collision (TTC), based on instantaneous positions and velocities of the vehicles (Vogel 2003). While in the speed control problem there is essentially a single target point, the more complex lane-keeping activity involves multiple conflict points and more complex vehicle kinematics.

The team focused on yaw velocity rather than the related variables of path curvature and lateral acceleration because of the importance of visual reference. Yaw velocity is directly available to the driver as the perceived angular rate of distant or peripheral objects across the field of view. Path curvature by contrast requires a constructive element as the driver "imagines" the path of the vehicle, something that is surely more appropriate to low speed maneuvering. Again, vehicle lateral acceleration is not a visual input, but rather a feedback for the lower-level manual control of the vehicle. Thus, the emphasis on yaw rate as the reference is based on its availability through visual feedback. This is analogous to what happens in vehicle stability control (e.g., Trachtler 2004)—vehicle yaw rate is directly measured and compared to a referencethough in this case, it is based on anticipated vehicle response to steering at the current speed. In this case path curvature is 
not directly measurable, and lateral acceleration is subject to many dynamic disturbances such as body roll; also, the lateral acceleration is dependent on location (whether that is at the driver's head or in a solid state electronic device). By contrast, the yaw rate is only sensitive to sensor orientation.

It is also worth noting that under ideal conditions of constant speed, minimal vehicle sideslip (when the vehicle is in a normal stable condition) and negligible body roll angle, the three variables mentioned (path curvature, yaw rate, and lateral acceleration) are simply proportional to each other. So under these simple conditions, any one of these variables might be used for the current purpose.

For any point on the road or lane boundary, we are to determine whether a yaw rate correction is needed to avoid going outside of the lane/road. If so, the yaw correction required is a measure of conflict. The maximum magnitude of all such corrections (left or right) is to be the conflict measure, though it is often of interest to analyze "worst right boundary case" and "worst left boundary case" in parallel. Additional information is relevant, namely, the distance and polarity (left, right) of any conflict point, as well as the horizon distance. The horizon distance is the maximum distance or headway for which, under ideal yaw rate, no conflicts occur. The horizon distance is a combined measure of position and direction error, as well as road geometry, and arises naturally out of the YRE analysis.

As mentioned, YRE and these associated measures are related to time to lane crossing (TTLC), but are expected to incorporate a greater degree of continuity and relevance into the control task. Unlike TTLC, the "angle of attack" of the lane excursion is implicitly included, so it potentially attaches due significance to how severe the predicted lane excursion will be, not just when it will be. For this reason, YRE is expected to be a superior combined metric of lane-keeping performance analysis than TTLC.

We now consider how to construct the yaw error criterion by using NDD. In Figure 7.1 we represent the lateral vehicle control relative to a single "conflict point" $P$. This is presumed to be on the right lane boundary, so the yaw rate (positive in the case shown, with the vehicle curving to the right) should be no more than for the critical case shown. The vehicle point $Q$

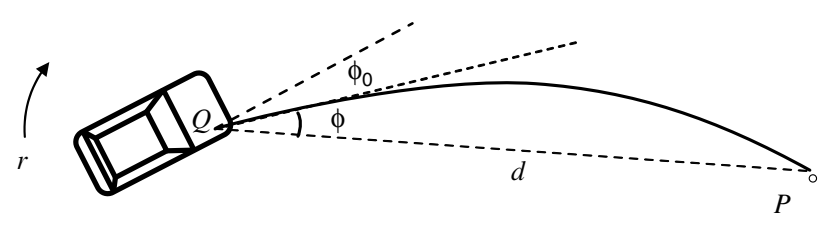

Figure 7.1. Turning kinematics (reference point $Q$ intersects with boundary edge point $P$ during a steady state turn). required to pass to the left of $P$, while here it just intersects with $P$. Using polar coordinates $(\phi, d), \phi$ is the azimuth angle and $d$ is the distance-to-target, both computed relative to the velocity vector at the reference point $Q$. This in turn is oriented at an angle $\phi_{0}$ relative to the vehicle axes, and if $Q$ is assumed to be at the outside edge of the front right tire, then $\phi_{0}$ is very roughly equal to the steering angle at the right front wheel.

The vehicle path is in the form of a circular arc, for which the essential geometry is represented in Figure 7.2. We find that the critical case occurs when the turning radius $R$ satisfies Equation 7.1

$\sin \phi=\frac{d}{2 R}$

which is equivalent to the yaw rate condition

$r=\frac{2 U \sin \phi}{d}$

where $U$ equals vehicle speed. Equation 7.2 defines the maximum yaw rate of the vehicle to avoid conflict with a right boundary point $P$.

When driving data are used, there is no direct information on all of the variables used in the above. The absolute coordinates of the boundary points are unknown, as are their relative locations to the vehicle. Therefore, they must be inferred from the lane tracker, which estimates lateral position and lane width. Note that while in principle GPS could be used, it is far from being accurate enough to give useful estimation of the lane-keeping performance, so this is not considered. The team proposes a method that is more realistic to estimate instantaneous value of YRE by using multipoint measurements of lateral lane position, vehicle yaw rate, and vehicle speed. Essentially this is to distinguish vehicle path curvature from highway curvature by using variations in lane position. The calculations and their derivation are somewhat messy (see Appendix D). However, they turn out to be entirely feasible. As a "free bonus," the YRE estimation technique provides a potentially useful estimation of underlying road curvature; this is based on vehicle yaw rate, but factoring out any lateral drift of the vehicle within the lane. Such estimation

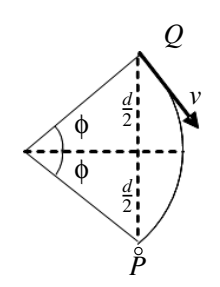

Figure 7.2. Essential geometry of steady state turning motion. 


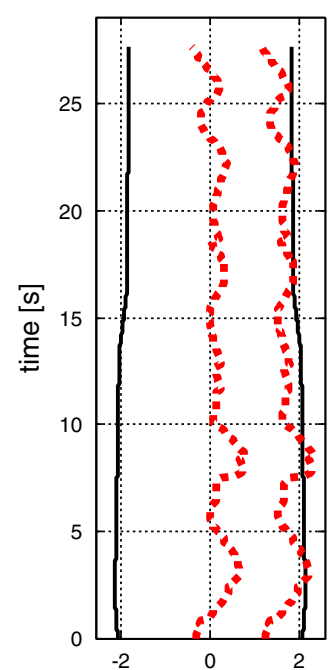

(a) distance [m]

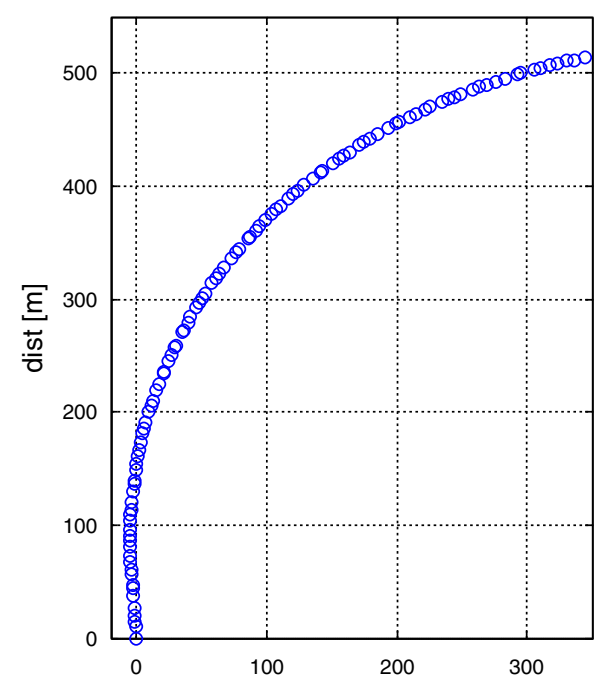

(b) dist [m]

Figure 7.3. The vehicle path for Event 1. (a) The dotted lines represent the left and right edges of the vehicle with respect to the center of the lane markings (solid lines). (b) The XY position of the vehicle in space.

is not applied in this project, but it is worth noting that it is available for future use.

The motivation for YRE estimation was to provide a robust and continuous measure of lane-keeping control performance. To test this idea, at least in an informal way, the team considered a small number of driving situations and compared YRE to TTLC and its reciprocal, inverse time to lane crossing (ITTLC). The estimation method described above was used to determine the YRE for driving events recorded in the UMTRI NDD. Event 1, depicted in Figure 7.3, shows a driver negotiating an on-ramp that is in the form of a right-hand curve.

The left plot shows the location of the left and right front wheels relative to the lane boundaries. Note that there is some variation in the lane width, but that most of the variations are in the dashed lines, which depict the outside edges of the front tires. This event shows a situation in which the driver maintained a position very close to the lane boundary with several excursions beyond the boundary. From video review, it appeared that the driver's attention was switching between reading a map and looking at the road ahead. The event represents an example of poor lane keeping. Figure 7.4a shows critical and actual yaw rate time histories, as well as lateral distance within the lane boundary (scaled by a factor 0.1 so that scales are reasonably consistent).

All conflicts for this event appear to be right side only, so the YRE in Figure 7.4b is positive whenever the current location and path predict at least one lane boundary conflict within the chosen time horizon ( 0.5 to $2 \mathrm{~s}$ ). Note that the YRE is always positive at the start of a lane excursion, and actually

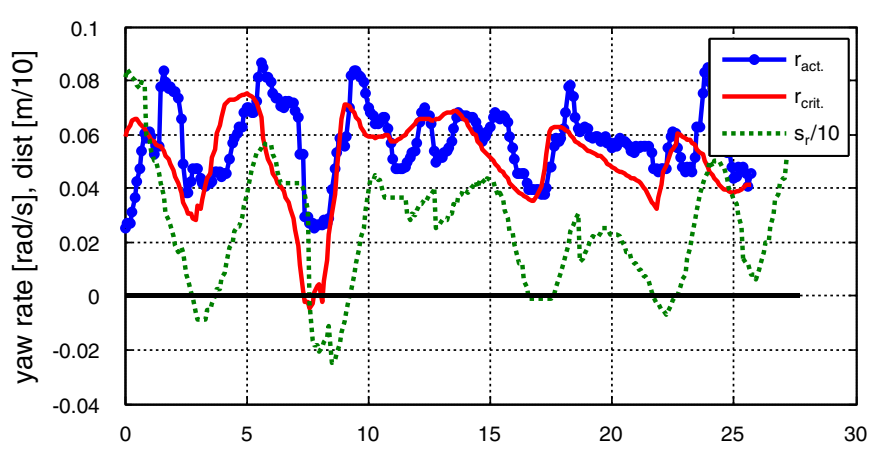

(a) time $[\mathrm{s}]$

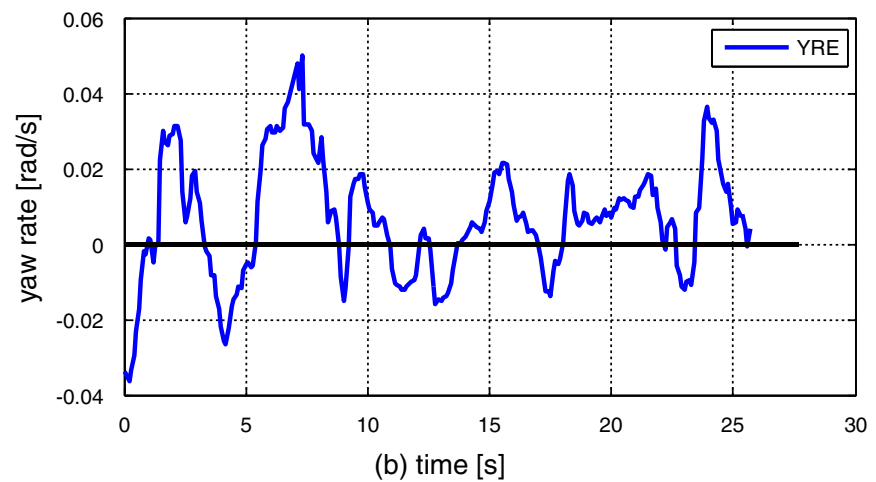

Figure 7.4. The yaw rate parameters for Event 1. (a) Actual yaw rate, critical yaw rate, and distance to right lane boundary. (b) The calculated YRE through the curve. 
always becomes positive before a lane excursion occurs. In this sense, as would be expected, YRE is predictive of each lane excursion.

Figure 7.5 shows YRE again (lower plot) together with TTLC in the upper plot and also its reciprocal, ITTLC, in the center plot. ITTLC might be preferred as a conflict metric since large values indicate proximity to a lane excursion, in contrast to TTLC, which is large when the vehicle is tracking the lane well.

The main features seen in Figure 7.5 are the great variations and major discontinuities in TTLC and ITTLC, as compared to the more continuous form of YRE. This suggests that YRE may connect more directly to the continuous steering control behavior of the driver, especially because lane crossing is not generally a catastrophic event and does not generate a panic response from the driver.

In Figure 7.6 this is tested informally by plotting steer response (upper curve) as well as YRE (lower curve). Each local peak of the YRE curve seems to coincide with a sharp negative slope in the steering, and this is the case at the YRE peaks at around $t=2,7,16$, and $24 \mathrm{~s}$; these sharp reactions seem to correlate with corrective actions by the driver in a way that TTLC, ITTLC, and even lane crossing in Figure 7.4a do not. The distracted driver in this event is not responding to YRE as it reaches positive values, but arguably when attention to the road coincides with a positive value of YRE.

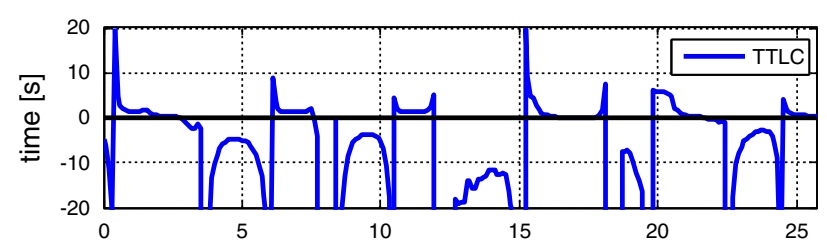

(a) time [s]

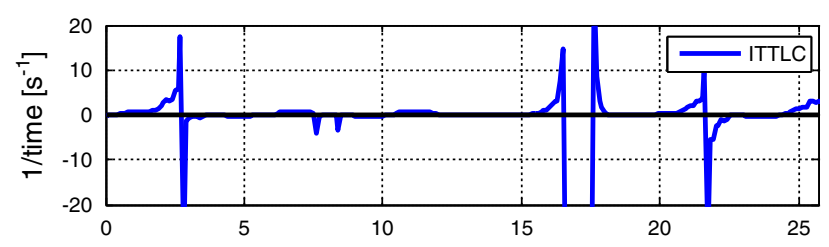

(b) time [s]

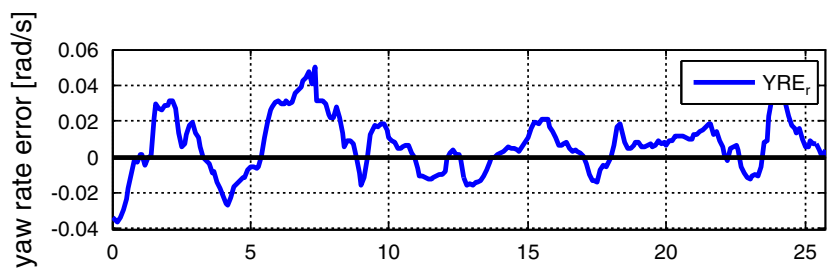

(c) time [s]

Figure 7.5. A comparison of the driver risk parameters for Event 1. (a) TTLC. (b) ITTLC. (c) YRE.

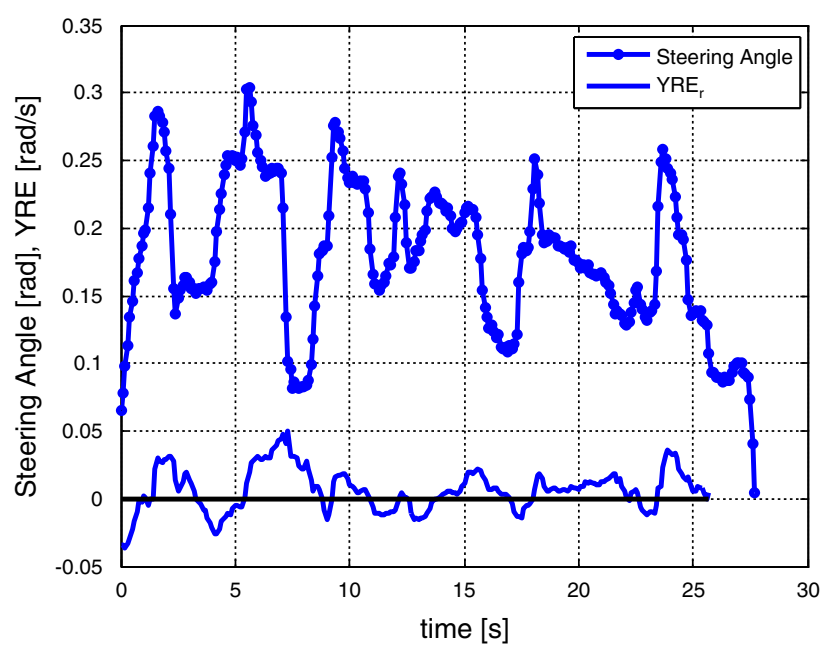

Figure 7.6. A comparison of the driver-controlled steering angle and the calculated YRE for Event 1.

The second event considered was a single boundary crossing followed by a correction back to the middle of the lane. The vehicle trajectory data can be seen in Figure 7.7. The event is somewhat simpler than Event 1, in that only one major excursion exists. Figure 7.8 shows the event in terms of yaw rate and critical yaw rate, and it is interesting that the conflict most heavily dominated by variations is the critical yaw rate rather than the actual yaw rate.

In the upper plot of Figure 7.8, the yaw rate exceeds its critical value at around $7 \mathrm{~s}$, while the first lane excursion takes place around $1 \mathrm{~s}$ later, again showing the predictive nature of YRE. In the lower plot, the YRE undergoes a correction at $t=10 \mathrm{~s}$ and from the previous analysis we would expect to see a sharp negative slope in the steering angle then.

Notice that in Figure 7.9, the previous comparisons with TTLC and ITTLC are consistent: the time-based metrics show large discontinuities, while YRE varies continuously and in a simple way during the event. YRE grows at a very roughly uniform rate until the correction is presumably applied at $t=10 \mathrm{~s}$, then decays uniformly until at around $12 \mathrm{~s}$, it is corrected again in the opposite sense. In Figure 7.10, a sharp negative slope is seen at $t=10 \mathrm{~s}$, and a positive slope steering correction takes place at $t=12 \mathrm{~s}$, as expected. Of course there are other steering corrections visible in Figure 7.6, and not all are directly predicted by conflicts with the right lane boundary, but perhaps some involve the right lane boundary. To this end, a modified plot of vehicle yaw rate plotted over the pair of critical boundary cases is considered below.

First, however, we consider a third example, also on a curved road section but one where there are no obvious lane boundary conflicts (Figure 7.11). It shows a nearly uniform distance from the car to the lane boundaries while negotiating the right-hand curve. Surely in this case the control loop is 


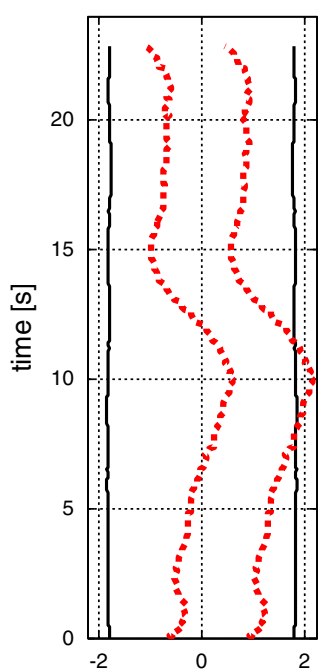

(a) distance [m]

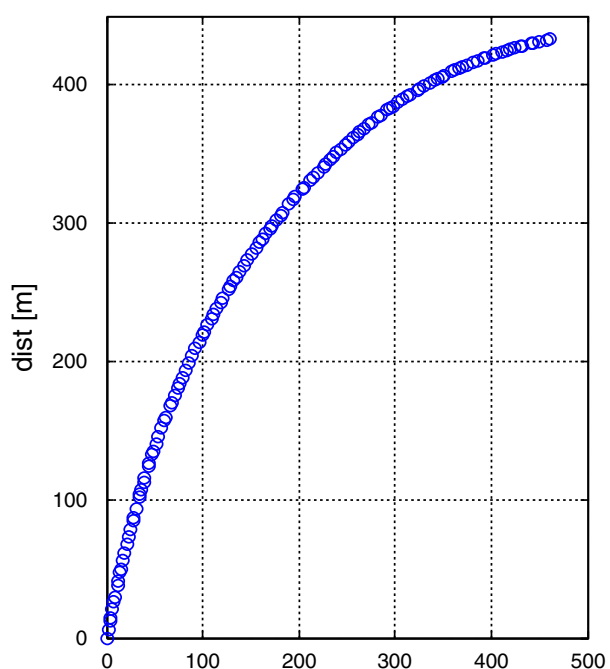

(b) dist [m]

Figure 7.7. The vehicle path for Event 2. (a) The dotted lines represent the left and right edges of the vehicle with respect to the center of the lane markings (solid lines).

(b) The XY trajectory of the vehicle.

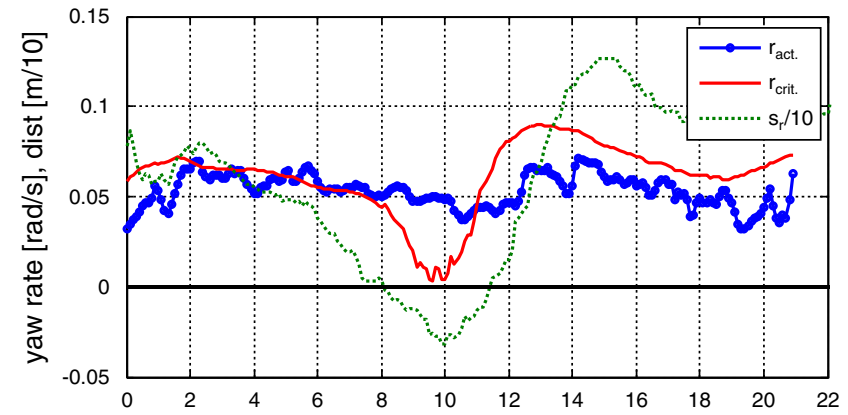

(a) time [s]

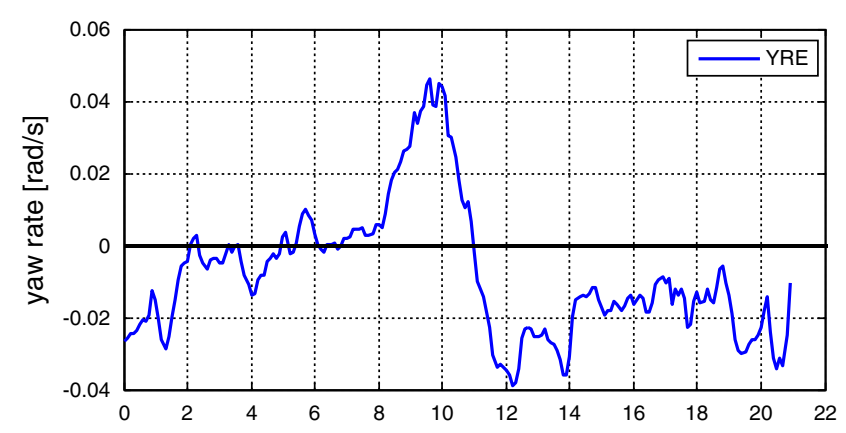

(b) time [s]

Figure 7.8. The yaw rate parameters for Event 2. (a) The actual yaw rate, critical yaw rate, and distance to the right lane boundary. (b) The calculated YRE through the curve.

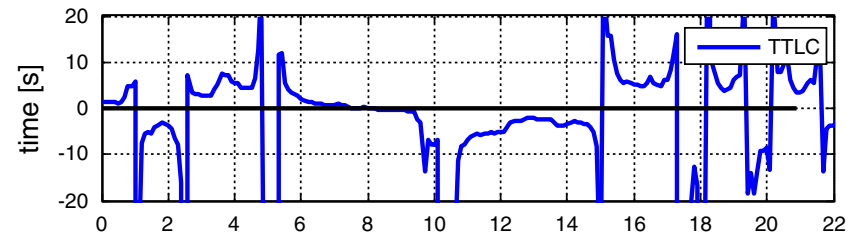

(a) time [s]

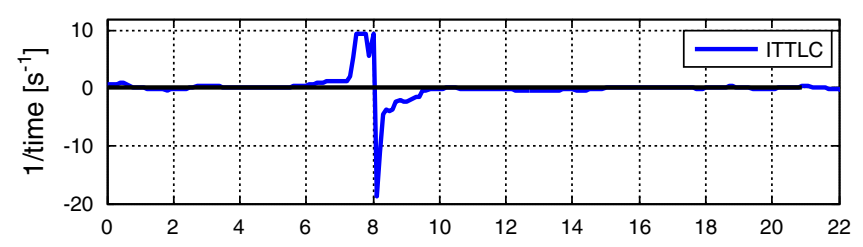

(b) time [s]

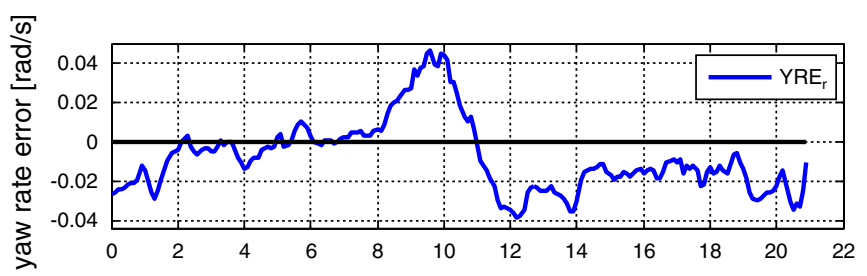

(c) time $[\mathrm{s}]$

Figure 7.9. A comparison of the driver risk parameters for Event 2. (a) The TTLC.

(b) The ITTLC. (c) The YRE. 


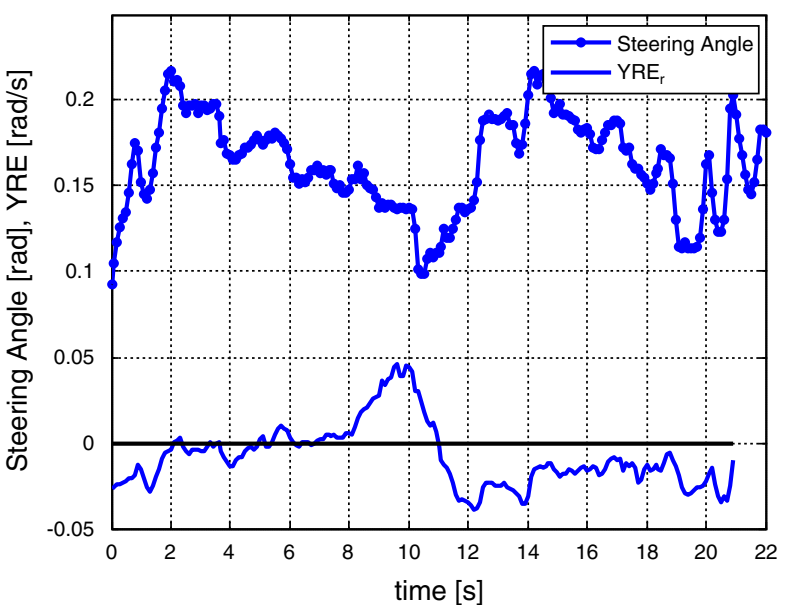

Figure 7.10. A comparison of the drivercontrolled steering angle and the calculated YRE for Event 2.

inactive, meaning that the driver has found a stable line and has no need to make multiple corrections to avoid boundary conflicts. Figure 7.12 appears to show otherwise. Again, we are plotting YRE for the right boundary and steering control actions. Far from being random or disconnected from the boundary conflict, the driver appears to be making regular steering corrections (negative slope interventions) whenever YRE approaches a critical (zero or positive) value.

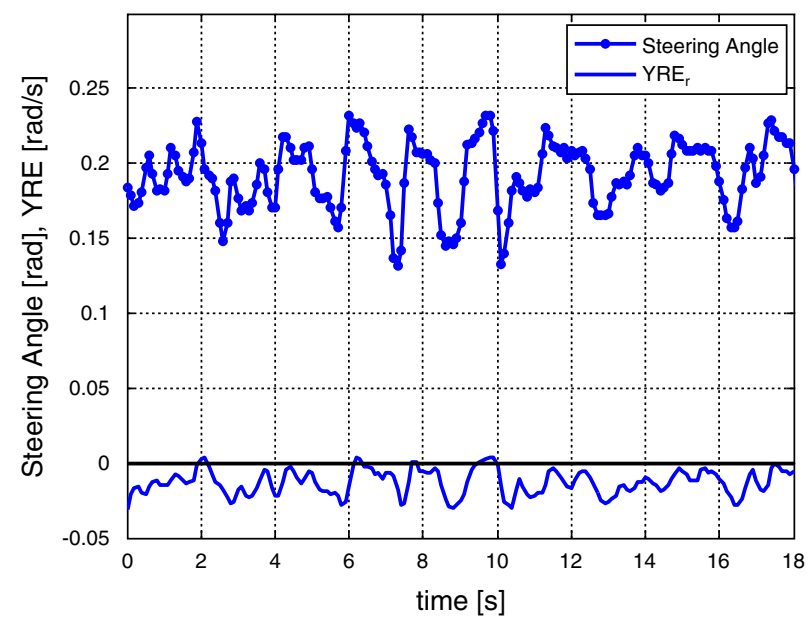

Figure 7.12. A comparison of the drivercontrolled steering angle and the calculated YRE for Event 3.

Figure 7.13 shows the yaw rate versus its two critical limits, where conflict avoidance takes the form

$r_{c, \text { left }}<r<r_{c, \text { right }}$

All three events are shown, but the most striking is for Event 3 in the lower plot: the vehicle appears to be controlled very precisely within the critical boundaries, with minimal

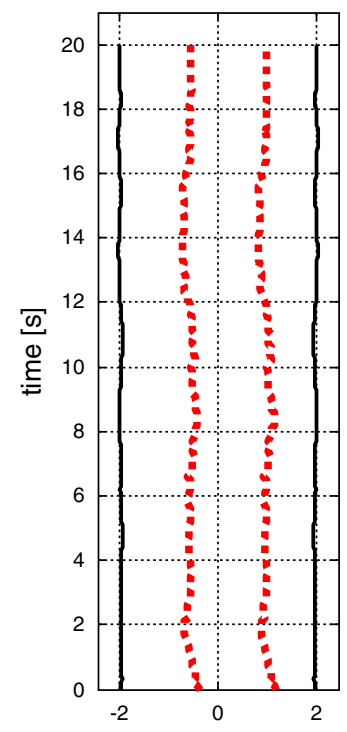

(a) distance [m]

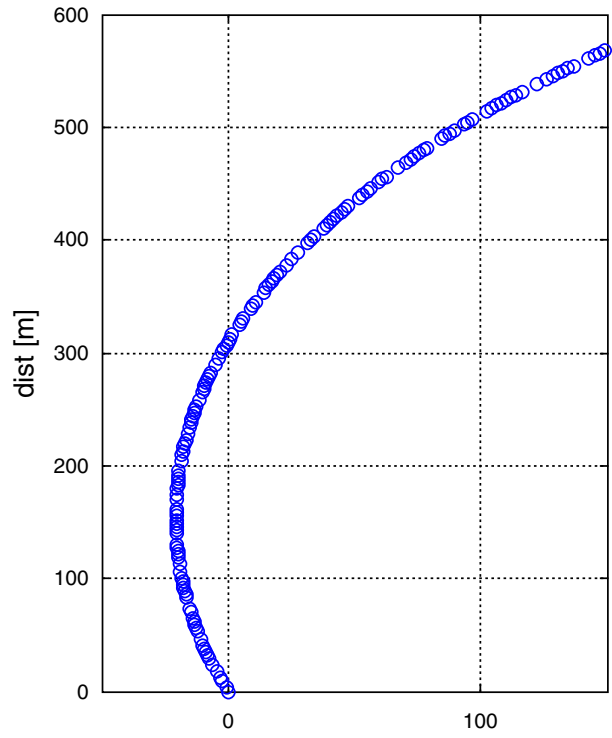

(b) dist [m]

Figure 7.11. Vehicle path for Event 3. (a) The dotted lines represent the left and right edges of the vehicle with respect to the center of the lane markings (solid lines). (b) The XY position of the vehicle in space. 


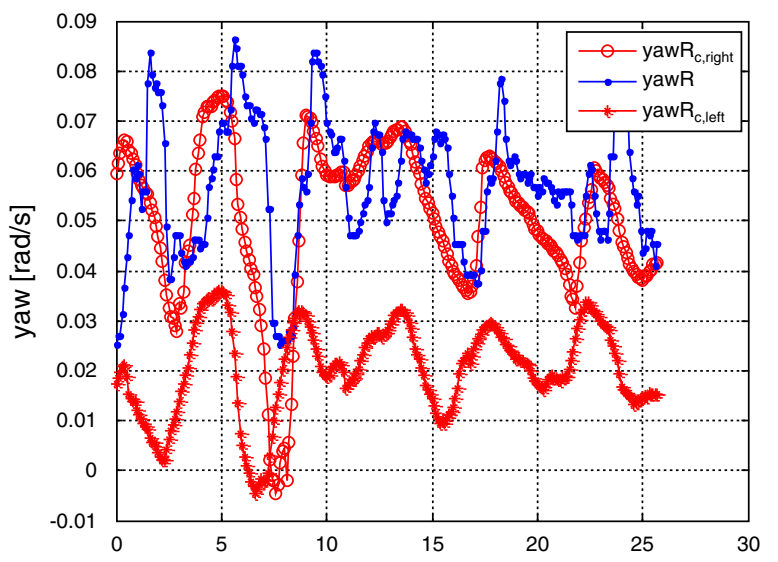

(a) time [s]

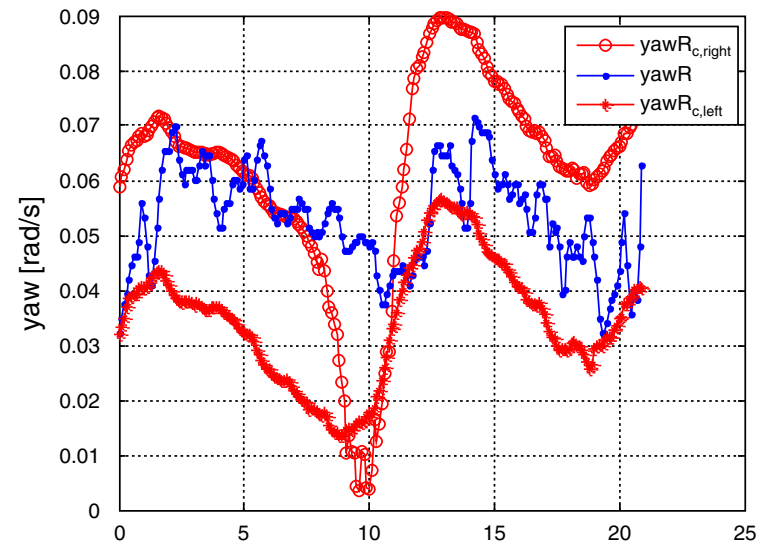

(b) time [s]

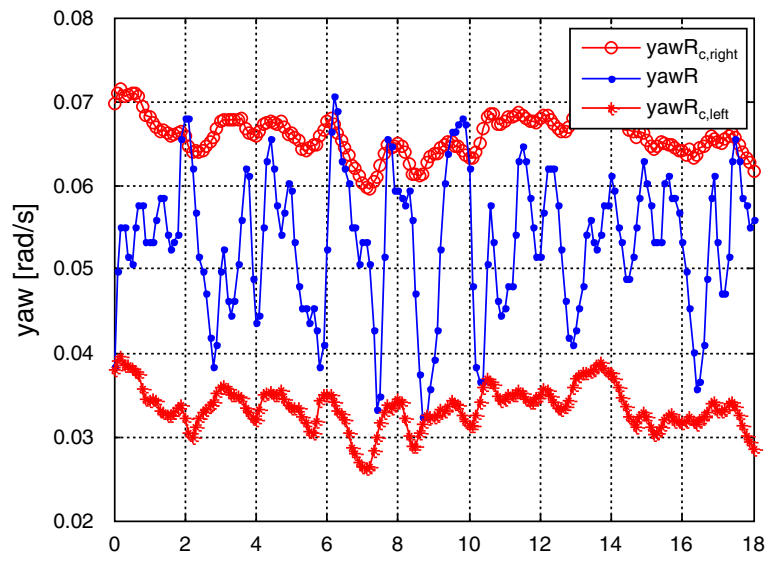

(c) time [s]

Figure 7.13. Comparison between the critical yaw rate for left and right boundary conflicts and the actual yaw rate for (a) Event 1, riding the right boundary; (b) Event 2, single boundary crossing with correction; and (c) Event 3, good lane following. overshoot but using the full range. Far from a stable "on center" steering control tracking the lane center, in "YRE space" the vehicle is bouncing quasiperiodically between its limits. If this interpretation is correct, the YRE provides a simple picture of lane-keeping control actions by the human driver. Turning to the center plot, where a single excursion event was seen, the degradation in control appears to be initiated as early as $t=3 \mathrm{~s}$ when the more stable "bouncing between limits" is interrupted. After the lane excursion is corrected, normal effective control appears to be regained at around $14 \mathrm{~s}$. Turning back to Figure 7.4a, this same interpretation seems reasonable from the within-lane drift: intuitively the driver is drifting right from about $t=3 \mathrm{~s}$, and only recovers full control at around $t=15 \mathrm{~s}$. The point here is that YRE seems to provide a direct measure of lane-keeping performance, and may even correlate with the error criterion active in the control loop of the human driver. In Figure 7.13a, it appears that the driver does not regain effective control of the vehicle throughout the $15 \mathrm{~s}$, and this is consistent with the distracted nature of the driving event. Finally in the upper two plots, left and right boundaries actually cross over, so no solution to the above equation actually exists. This interesting situation is briefly considered below.

As intended, we have defined and evaluated a YRE criterion as the basis of potential new surrogates for single-vehicle road departure crashes. The measure appears to be strongly connected to lane-keeping control, and has several advantages over similar but less clear measures. Note that

- YRE behaves in a continuous way, even when lane boundary crossings take place, and this is not the case for TTLC and its reciprocal (ITTLC).

- YRE excursions correlate strongly with rapid steering interventions by the driver, especially when the driver is providing effective control of lane position.

- When left and right critical yaw rate boundaries are considered simultaneously, the normal effective control of lane position appears to operate to constrain between the crucial limits.

- YRE may be a useful predictor of actual lane excursions, but, more important, it seems to provide a strong indicator of degraded or ineffective lane keeping.

In Events 1 and 2, the lane excursions appear to induce an impossible situation for the driver: the left and right limits cross over. This is most easily seen in Figure 7.13b, where crossover takes place between approximately $t=8$ and $t=12 \mathrm{~s}$. From Figure 7.7a and Figure 7.8a, this corresponds to the vehicle being outside the lane boundary; the steering task changes from lane keeping to lane recovery, though from 
Figure 7.10, the reaction seems to be consistent with a single sharp correction to divert the YRE to a correct linear rate of descent, followed by a second sharp correction in the opposite direction at around $t=12 \mathrm{~s}$. Thus it seems that the crossover is not a major factor to the driver, who perhaps applies focus to one boundary at a time.

The preceding results were based on arbitrarily chosen events. There was no selection procedure adopted other than to find events from lane position typical of (a) an extended period of degraded lane control, (b) a single event lane excursion error, and (c) well-controlled lane keeping. Informal reviews of many such events appear to show that YRE control relationships in lane keeping are robustly followed by most drivers in most conditions. Further work will be needed to expand the number of events and attempt to quantify formally and accurately the relationships hinted at in the three events presented. In Chapter 8, the section Road Boundary Change and Yaw Rate Error briefly considers the application of YRE to "road widening" scenarios, where the right lane boundary becomes ambiguous and one might expect lane-keeping control to be disturbed. Otherwise, the analysis of YRE is limited to the brief exploration presented above. 


\section{онартен a \\ Orthogonal Studies}

The emphasis of this project has been on creating and demonstrating feasible analysis methods for the SHRP 2 naturalistic driving study, using limited data that already exists. While the main thrusts of this analysis have been in Bayesian estimation and extreme value theory, the research questions posed can be addressed by other techniques. In this chapter the research team proposes simple additional relevant analysis techniques that can be used to resolve the data in different (orthogonal) directions. The first of these techniques considers how existing GIS tools for the analysis of spatial clustering may be directly applied to road departure crash data, with the aim of suggesting crash patterns and hence formulating hypotheses for deeper analysis. The second technique relates to driver distraction and surrogacy by comparing surrogate event rates in episodes of driving while on and off a cell phone. The third technique compares a measure of control disturbance (YRE) in the presence of particular highway features, based on the tentative hypothesis that disturbed control is more likely when lane or road boundaries change, for example, when the road widens near the entrance to a side-street. As with the main analysis approaches that have been used, the aim is to propose and test viability for application to the future SHRP 2 driving database and not to answer specific research questions based on the limited data used.

\section{Road Departure Crash Spatial Analysis}

Examining common features of spatial clusters of crashes, or hot spots, can be useful in identifying patterns in the occurrence of such events. The common features of the hot spots can be related to roadway characteristics or to driver behaviors captured in NDD, or to both, and can be informative in developing hypotheses for further analyses. For example, examination of roadway data from the hot spots may suggest roadway features associated with the crashes and may provide a nonparametric indicator of the existence of surrogates.
Examination of driving data at the hot spots may suggest patterns or particular driver behaviors for deeper analyses.

What appear to be concentrations of crashes, however, could simply be a result of random occurrences, and looking for commonalities in these random groupings would be a wasted effort. Spatial analysis tools allow researchers to identify clusters that constitute statistically nonrandom, spatially dependent hot spots. In this orthogonal study, ArcToolbox spatial analysis tools are used to identify nonrandom spatial clusters of run-off-road crashes. The development of comprehensive information about the hot spot clusters through the joining of spatially referenced crash locations, roadway, and roadway characteristics is also demonstrated.

In spatial analysis, hot spots are formally defined as clusters of points with values higher in magnitude than one would expect to find by random chance. The spatial analysis of 7,924 road departure crashes in Oakland County, Michigan, from 2001 to 2005 was completed by using the Getis-Ord $\mathrm{G}_{\mathrm{i}^{\star}}$ statistic. The Getis-Ord $\mathrm{G}_{\mathrm{i}^{*}}$ statistic is a well-known method for establishing local spatial dependencies that may exist and one of the six statistics designed to address local relationships among geo-referenced data (Getis and Ord 1996).

The conceptualization of spatial relationships used for weighting the crash data was defined by using the inverse and Euclidean distances. The threshold distance chosen for the calculation was zero. The inverse distance method gives more weight to those crash locations that are close to each other and less weight to those farther apart. The Euclidean distance models the shortest path between events, and a threshold distance of 0 ensures that no points are dropped from the analysis. The road segment crash rate was selected as the random/ independent variable or our exposure value.

Given a set of weighted data points, the model generated a $Z$-score for each segment along which a crash occurred. Hot spots are indicated by a $Z$-score greater than +1.96 or locations with a high crash-rate cluster. The hot spot location has a high crash-rate value and its neighbors also have high crash-rate 
values or higher than would be expected by random chance. These hot spots indicate nonrandom, spatially dependent crash-rate locations.

Running the model resulted in only a few (397) of the 7,924 crashes having a $Z$-score greater than +1.96 or an indication of spatial dependency. There were no $Z$-score values less than or equal to -1.96 , which means that no "cold spots" were found. Cold spots are locations where low crash rates are surrounded by locations with similarly low crash rates.

The orange dots on the top of Figure 8.1 indicate the hot spot locations. The aerial image zooms in on a hot spot cluster. The crashes, digital base map segments (the blue line), and signage can be viewed as a layer on the aerial photograph. The data table illustrates the selected HPMS road segment data that were associated with crashes.

Although this hot spot is located near an intersection, the crashes considered here are single-vehicle run-off-road crashes. Further analyses of the crash data or of driving data might reveal some patterns worthy of further pursuit. However, it was not the intention of this exploratory study to establish common factors of the crashes or go into further analysis, but to demonstrate the use of spatial analysis tools and to suggest that such an investigation would be fruitful. The results of any such microscopic analysis may suggest possible common elements and help formulate a hypothesis for more formal study.

\section{Cell Phone Use}

Engaging in secondary tasks while driving taxes a driver's attention resources, leaving less for the driving task. In many normal driving situations the attention demand for driving is met even with secondary tasks without crashes or other incidents. However, whenever attention demand exceeds available attention resources, the risk of a crash increases (Eby and Kostyniuk 2004a). Carrying on a cell phone conversation while driving has been shown to negatively affect driving

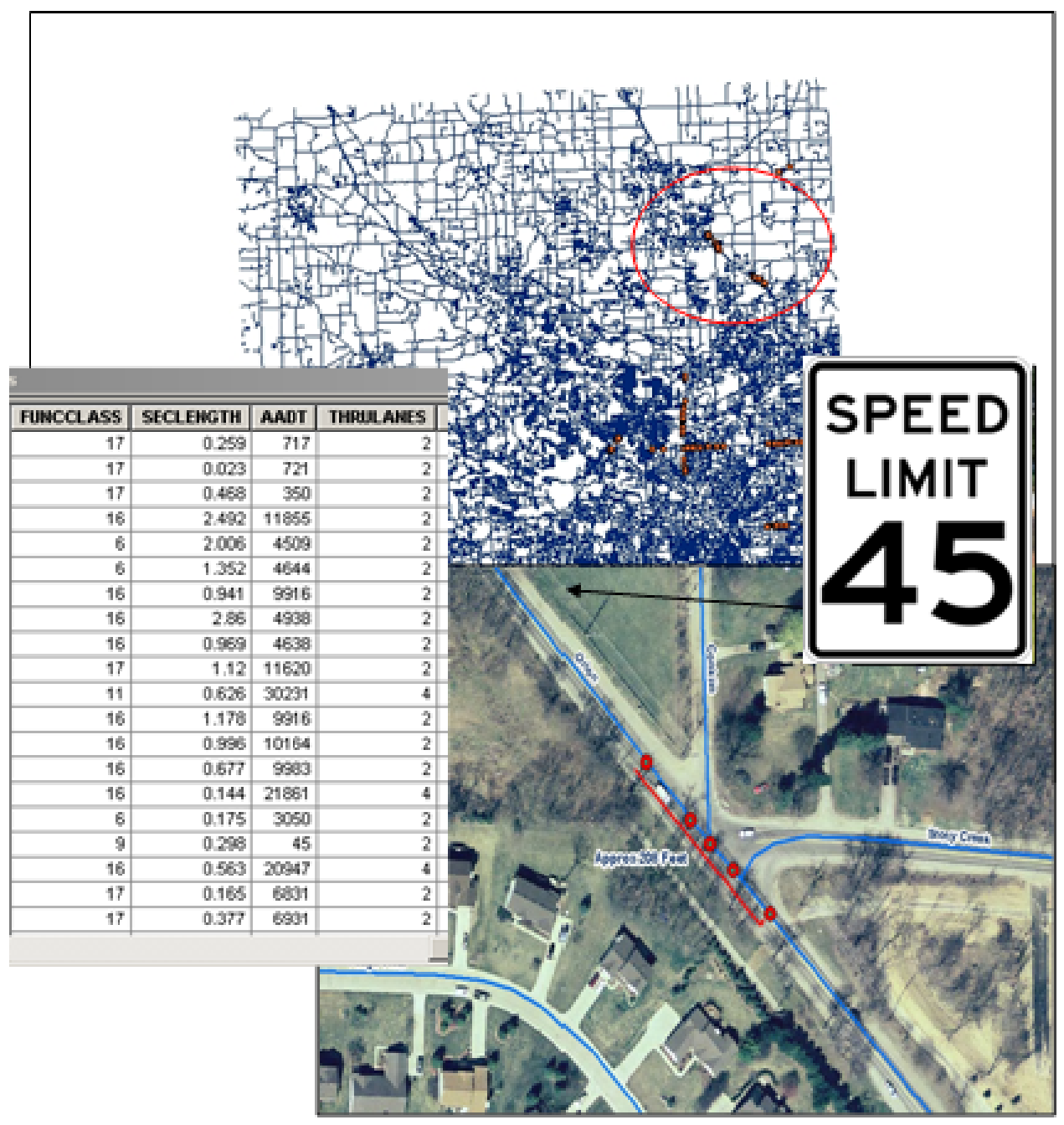

Sources: Google Earth, ArcMap XY plot using project data, and Uniform Traffic Control Manual.

Figure 8.1. Clusters of run-off-road crashes, Oakland County, Michigan. 
performance in many simulator studies (Caird et al. 2004; Hoerrey and Wickens 2004). There is also much anecdotal evidence of unsafe driving behavior of drivers on cell phones. This suggests that the effectiveness of tactical and operational aspects of the driving task is reduced when a driver is engaged in a cell phone conversation. Furthermore, if this is true, then there should be some evidence of disturbed control in the vehicle kinematic history of the driving episode.

This study explored LDW alerts as a marker for disturbed vehicle control in a comparison driving with and without a cell phone. Episodes of cell phone use were identified in the RDCW naturalistic driving database by visual review of face videos of the first week of driving for all subjects. Comparison episode periods were selected for each cell phone episode of each driver randomly from the same trip and on the same road type.

On average, each subject in the RDCW study drove the vehicle on 32 trips in the first week of driving. Of the 78 subjects, 61 used the cell phone in the first week, spending an average of $1,150 \mathrm{~s}$ on that activity. The number of cell phone episodes per driver ranged from 1 to 64, with an average of 11.6 episodes. The duration of the cell phone episodes ranged from 4 to $818 \mathrm{~s}$, with an average of $99 \mathrm{~s}$.

Of the 61 subjects, 25 (41\%) did not trigger an LDW alert while on a cell phone or during the comparison periods. Of the 36 drivers with LDW alerts, 18 (50\%) triggered alerts only in comparison periods (i.e., while not on a cell phone), $4(11 \%)$ triggered alerts only during cell phone episodes, and $14(39 \%)$ triggered alerts in both cell phone and comparison episodes.

The overall LDW alert rate for each driver was developed from the cell phone usage on all of his or her trips, and the comparison rate was developed from all the comparison periods. Of the 14 drivers with LDW alerts in both the cell phone episodes and in the comparison periods, the rate of alerts during cell phone periods was greater than the rate for the comparison period for eight drivers and less than the rate for six drivers.

The exploration of LDW alerts and cell phone does not show a greater rate of LDW alerts while drivers are on cell phones. Indeed the indications are that in general, drivers are more likely to receive an LDW alert while not using a cell phone than while on a cell phone.

Because YRE appears to be a useful metric for lane excursions, the team examined its applicability to this situation also. YRE was calculated for episodes of cell phone use and comparison periods for several subjects. This exercise also did not show much difference between driving periods with cell phone and without cell phone usage.

While it seems that there should be some indication of a deterioration of vehicle control while the driver is engaged in a cell phone conversation, this approach was not able to identify it. A different analysis of secondary tasks in the same RDCW data by Sayer, Denvonshire, and Flannagan (2005) concluded that drivers select the time and conditions for their cell phone conversations and that there is a large amount of variability in the behavior between drivers, and even of the same driver on different days.

Studies with tight experimental design and controls will be challenging at best with natural use driving data. A different approach might be more suitable for analyzing the effect of cell phone use (or other secondary behaviors) on driver performance in natural use data. Rather than designing a study around episodes of cell phone use, one could design a study around the candidate marker of disturbed control (i.e., YRE or LDW), identify events or conditions in which critical values of these markers occur, and then examine the environmental and driver conditions that were present.

\section{Road Boundary Change and Yaw Rate Error}

Chapter 7 introduced the YRE estimation and explained how it might be used to determine the lane-tracking performance of drivers. This short study investigated the possibility that a change in road geometry, in the form of a deviation in the perceived road boundary, would influence the lane-tracking performance. The YRE was calculated for multiple passes past a point where the road provides deceleration/acceleration lanes (or generous turning radii) at $\mathrm{T}$-intersections and compared that to the YRE for travel with uniform boundaries.

Ten intersections were selected for analysis based on a random sampling of satellite images, and of these, two were traversed multiple times by various drivers in the NDD. Figure 8.2 shows satellite images of the two points used in the analysis. Notice that both points display a road widening situation for only one direction of travel, which enabled the traversals in the opposite direction to be used as a baseline for comparison. Point 1 had 35 traversals by four unique drivers, 23 in the direction with the widening and 12 that could be used for a baseline. Point 2 had 95 traversals by 10 unique drivers, 56 in the direction with the widening and 39 baseline passes in the opposite direction.

The YRE was calculated for $5 \mathrm{~s}$ before and after the middle of the intersection for each pass in both directions. Figure 8.3 shows the standard deviation of the YRE for each 10-s pass. Even without formal statistical analysis, in each case there appear to be no systematic differences between the underlying samples shown in red and blue. This is not particularly surprising, given the small size of the samples. The implication is that the general driving pattern is unaffected by the road boundary change. On the other hand, the break, or deviation in the perceived road boundary, may occasionally provide an outlier caused by a corresponding disturbance in the 


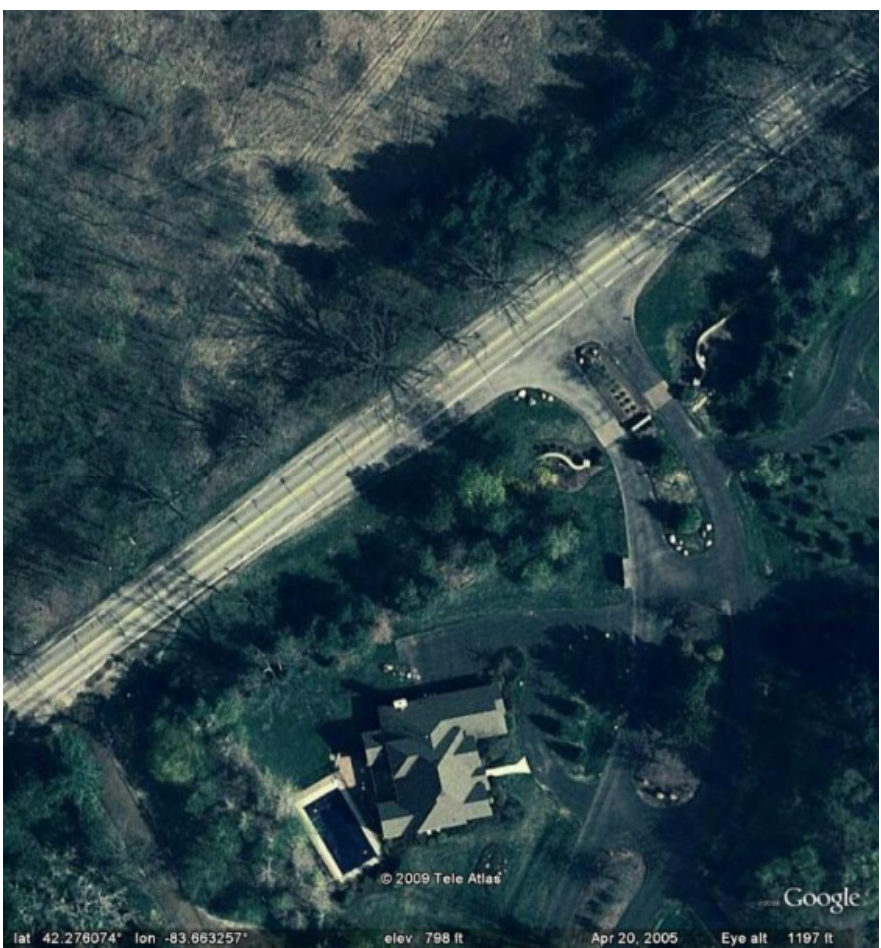

(a)

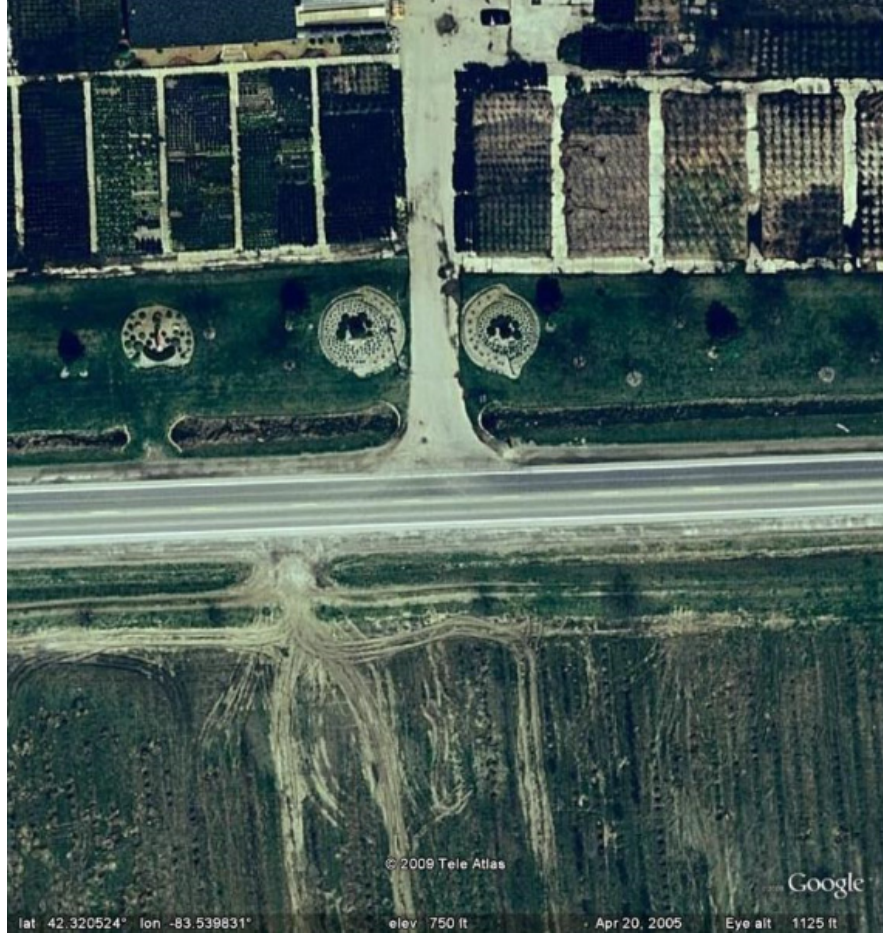

(b)

Source: Google Earth

Figure 8.2. Road widening on right boundary for (a) Analysis Point 1 (42.276308, -83.662979) and (b) Analysis Point 2 (42.320524, -83.539831).

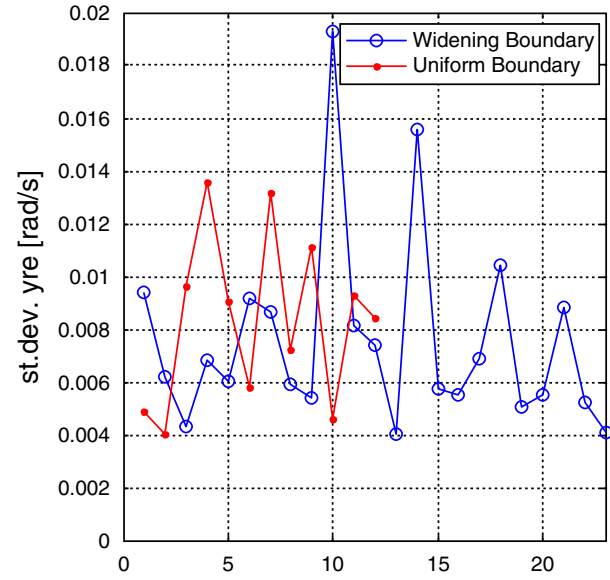

(a) Point 1 Driver Pass

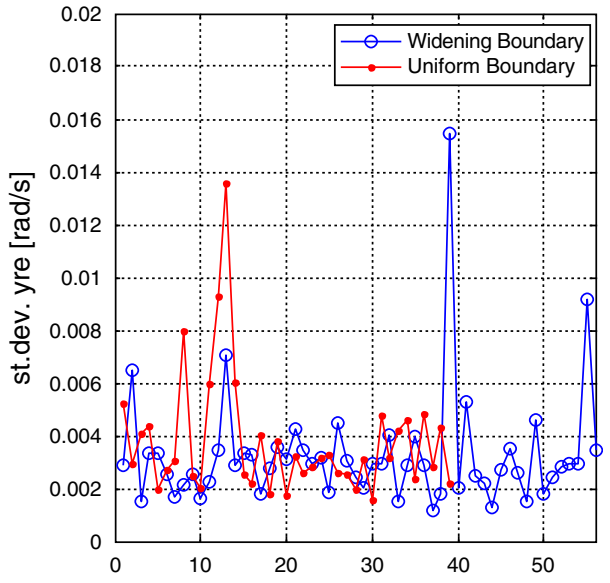

(b) Point 2 Driver Pass

Figure 8.3. Standard deviation of the estimated YRE for travel in the direction of the broken boundary and complete boundary (baseline driving conditions) for (a) Analysis Point 1 and (b) Analysis Point 2. 
lateral control. The fact that the blue outliers here are the more extreme is somewhat supportive of this idea, but clearly more data would be needed to provide definitive results. YRE is preferred to simpler lane-tracking measures, given its apparent strength of connection to control disturbances, and extreme values might be more productive than using standard deviations. To gather more data, a pool of corresponding sites could be used for aggregation, or it might be that in the future largescale driving study, a larger sample of relevant traversals could be found. The point here is that a study relating specific safetyrelated driver behavior to highway features appears feasible, but that a credible connection to disturbance in control is an essential ingredient.
While the directional differences are small and inconclusive, differences in location are very clear. Comparing Point 1 and Point 2 for the mean levels of YRE (aggregated over both directions), for Point 1 there is a mean \pm one standard deviation of $0.0078 \pm 0.0035 \mathrm{rad} / \mathrm{s}$, while this measure for Point 2 (both directions) is $0.0035 \pm 0.0022 \mathrm{rad} / \mathrm{s}$. The YRE calculation demonstrates sensitivity to the differences in lane-tracking performance between different locations. Clearly there is a difference between the driving that occurred on Points 1 and 2. Figure 8.3 shows that the driving near Point 1 had a larger variation in YRE than did the driving near Point 2, indicating less uniform lane tracking and more driver corrections for Point 1 . The reasons behind this difference have not so far been investigated. 


\section{онАРтE⿱ \\ Transferability to Other Data Sources}

The following sections describe use of the geospatial methods described previously to data mine field operational test (FOT) data in support of work to identify potential crash surrogate measures. In the previous sections, the driving data was collected during the University of Michigan Transportation Research Institute (UMTRI) FOTs. The roadway data were collected from various southeastern Michigan data sets. In this chapter, similar geospatial techniques will be tested against different data sets to explore the feasibility of transferring techniques to researchers with different data. Specifically, the purpose here was to demonstrate the same GIS-based data preparation methods using different naturalistic and roadway data.

\section{Data Used for Testing}

The naturalistic driving data (NDD) used in this demonstration were collected primarily in Virginia during the 100-Car Naturalistic Driving Study (Dingus et al. 2006). The roadway data used include databases from the Virginia Department of Transportation (VDOT) and the Federal Highway Administration (FHWA). The VDOT data were provided by staff in the VDOT Transportation Engineering Division, and the FHWA data were provided by the FHWA Office of Highway Policy Information.

\section{Crash Data}

VDOT crash data containing information recorded in police crash reports were used. These data include the latitude and longitude, date and time, jurisdiction, collision type, weather condition, surface condition, and lighting conditions of the crashes. Additionally, crashes from the 100-Car Study were used. Because the UMTRI method is intended to be used to study run-off-road crashes, only the crashes in the 100-Car Study that were classified as run-off-road crashes were used in this work. Of the 69 crashes in the 100-Car Study data set, 26 were run-off-road crashes. One of those crashes did not have valid GPS data, leaving 25 crashes available for use in this work.

\section{Highway Data}

The VDOT road data are geospatial, meaning that they include geographic location and shape information that can be mapped using computer software. Each road in the Virginia road network is made up of multiple segments of varying lengths. These segments are represented by lines in a data file, which indicate the centerline of the roadway. The centerline data also contain fields that hold attributes describing the section of roadway represented by each line. The attributes contained in these fields include the name of the road, the route number, the beginning and ending mile points, route category, and jurisdiction (i.e., county, city, or town), and segment length.

Highway Performance Monitoring System (HPMS) data were also used. FHWA gathers the data from each of the states annually and maintains the HPMS database. This database includes descriptive information for individual segments of the national highway network. The attributes available for each segment include information such as the state and county where the segment is located, the AADT, the length of the segment, and the number of through lanes. These data were delivered in a comma-delimited ASCII text file and are not geospatial.

A list of the 20 road segments in northern Virginia with the most vehicle fatalities and injuries during the years 2002 to 2004 was also obtained from VDOT. This list identifies those crash hot spots by route number and the beginning and ending mile points. It also includes descriptive attributes such as segment length, the number of crashes in the corridor, the number of those crashes that included an injury, the number of crashes with a fatality, and the crash rate. These data are not geospatial, and the segments do not necessarily correspond to the individual road segments in the VDOT centerline data representing the Virginia road network. 


\section{Software Tools}

The geospatial procedures used to prepare the naturalistic data for further analysis are dependent on the functions of GIS software. GIS encompasses a number of computer data management systems that specialize in geographic data and can be used to store, graphically represent, or analyze geographic data. ArcMap 9.3 from Esri with an ArcInfo license was used here.

Structured Query Language (SQL) was used to query relational database tables that were stored in a Microsoft SQL Server 2005 environment. SQL queries were executed in SQL Server Management Studio and in ArcMap.

\section{0-Car Naturalistic Data Description}

The time series data stored in the 100-Car Study include numeric measures such as vehicle speed, range to other vehicles, geographic location, lateral acceleration, longitudinal acceleration, and yaw, as well as video of the driver and surrounding environment. The 100-Car data describe what is occurring over time, both in noncrash situations and during actual crashes and near crashes. Within these data, epochs of driving can be located and analyzed further to identify how people drive in geographic areas of interest and in situations such as during the moments just prior to a crash, to identify what factors may contribute to crashes, and to perform detailed analyses of what occurred during these crashes. In this case, driving through road segments with high numbers of crashes can be located for further analysis.

\section{Comparing 100-Car Data and Anticipated SHRP 2 Field Data}

In many ways, the 100-Car data resemble the anticipated SHRP 2 field data. The majority of the measures available in the 100-Car data, and described in the previous section, will also be present in the SHRP 2 field data. Some additional measures are anticipated in the SHRP 2 data. For example, some roadways will be mapped in greater detail using a roadway measurement van. An automated method for monitoring the driver's gaze is also anticipated in SHRP 2. Latitude and longitude values collected by GPS are present in 100-Car data and will be available and stored in a database format in SHRP 2 data. These values will permit geospatial-related analyses to be conducted on the SHRP 2 data.

\section{Method}

The different data sources each have their own possibilities, but when they are joined, richer queries and analyses become available. Using spatial joins available in GIS and typical SQL joins based on fields in a database table, the data from the different sources were joined. The crash data were joined with the road data, which allowed counting crashes on each road segment in the Virginia highway network. After joining the VDOT road data and HPMS data, more attributes, such as number of lanes and AADT, were associated with the road segments in the VDOT data. Finally, naturalistic driving data were joined with their corresponding road segments. This process is described more completely in the following sections.

\section{Associate Road Attributes and Crash Statistics for Road Segments}

The first portion of this effort was to establish that state crash statistics could be associated with state roadway segment data. This processing will be of use in computing segment crash rates using whatever methods are appropriate for a given research question. Using GIS allows data to be joined by using spatial information. In a spatial join, data are joined based on geographic location instead of using attributes from columns within a table. The GPS data from the VDOT run-off-road crashes were plotted in a layer in GIS along with a layer of VDOT highway centerline data. The features in the two layers were then spatially joined. The result was a geospatial data set that can be plotted in GIS or queried like a table in a relational database. For this demonstration task, a query was written to count crashes that have occurred on each road segment in the VDOT road data and to identify the totals by route number. Values from a sample of 15 of these segments are shown in Table 9.1.

Table 9.1. Sample of Road Sections with Number of Crashes from VDOT Roadway and Crash Data

\begin{tabular}{|l|c|c|c|c|}
\hline $\begin{array}{l}\text { Route } \\
\text { Number }\end{array}$ & $\begin{array}{c}\text { Begin } \\
\text { Mile Point }\end{array}$ & $\begin{array}{c}\text { End Mile } \\
\text { Point }\end{array}$ & $\begin{array}{c}\text { Number of } \\
\text { Crashes }\end{array}$ & $\begin{array}{c}\text { Section } \\
\text { Length }\end{array}$ \\
\hline 6608 & 0.8 & 0.84 & 3 & 0.04 \\
\hline 7100 & 16.25 & 17.31 & 54 & 1.06 \\
\hline 6608 & 2.75 & 2.78 & 3 & 0.03 \\
\hline 6608 & 3.63 & 3.68 & 1 & 0.05 \\
\hline 7900 & 0 & 0.27 & 215 & 0.27 \\
\hline 6608 & 1.38 & 1.45 & 2 & 0.07 \\
\hline 168 & 6.78 & 6.87 & 4 & 0.09 \\
\hline 1112 & 0 & 0.16 & 44 & 0.16 \\
\hline 7100 & 22.6 & 22.99 & 11 & 0.39 \\
\hline 7100 & 23.89 & 23.96 & 26 & 0.07 \\
\hline 161 & 10.05 & 10.26 & 68 & 0.21 \\
\hline 7100 & 13.15 & 13.52 & 59 & 0.37 \\
\hline 6608 & 2.89 & 2.92 & 1 & 0.03 \\
\hline 7100 & 20.44 & 20.8 & 11 & 0.36 \\
\hline 8763 & 0 & 0.07 & 48 & 0.07 \\
\hline
\end{tabular}




\section{Joined VDOT Centerline Data and HPMS Data}

To investigate the roadway as an explanatory factor, the HPMS data can be used. These data include a number of attributes that could reveal contributing factors in crashes. As stated earlier, the HPMS data do not include geospatial information. However, FHWA has implemented the linear referencing system (LRS), which creates a unique identifier for each road segment based on the county where the segment is located, the route number, and the milepost marker. There is some flexibility in the system that allows each state to customize the numbering system to accommodate the needs of that state. By using this unique LRS ID, the data contained in the HPMS database were joined with geospatial centerline data provided by VDOT.

This join was accomplished by using SQL. In addition to their normal use with relational database tables, SQL queries can also be used with geospatial data sets. This allowed each segment in the VDOT geospatial data to be joined with its corresponding segment in the HPMS data on the basis of their shared identifying attributes (i.e., county, route number, and mile points). For this work, the joining required a twostep process. In the first step, an initial join was done in SQL Server Management Studio, where joins on multiple fields were possible. Then, a simpler join was done in ArcMap to join the result of the first join with the geospatial data from the original VDOT data. It is believed that the second step would be unnecessary by taking advantage of geospatial data types, although that belief was untested in this work. By joining the HPMS data with VDOT's road centerline data, an enhanced geospatially referenced layer was created that allowed use of GIS functionality. An example of the type of integrated data set made possible through this join is shown in Table 9.2.

In Table 9.2 the columns labeled Section Length, AADT, Present Serviceability Rating (PSR), Number of Lanes, High Occupancy Vehicle (HOV), and Truck Route are collected from the HPMS data.

\section{NDD through VDOT Hot Spots}

The previous sections have demonstrated the processing steps that would be used by researchers in quantifying elements of high crash-rate locations. A number of different approaches could then be used for locating segments that are likely to provide guidance in identifying crash surrogates for a specific crash type. Once these locations are identified, the objective

Table 9.2. Sample of Road Sections with Additional Attributes Joined from HPMS

\begin{tabular}{|l|c|c|c|c|c|c|c|c|c|}
\hline $\begin{array}{l}\text { Crash } \\
\text { ID }\end{array}$ & $\begin{array}{c}\text { Route } \\
\text { Number }\end{array}$ & $\begin{array}{c}\text { Begin Mile } \\
\text { Point }\end{array}$ & $\begin{array}{c}\text { End Mile } \\
\text { Point }\end{array}$ & $\begin{array}{c}\text { Section } \\
\text { Length }\end{array}$ & AADT & PSR & $\begin{array}{c}\text { Number } \\
\text { of Lanes }\end{array}$ & HOV & $\begin{array}{c}\text { Truck } \\
\text { Route }\end{array}$ \\
\hline 11 & 267 & 11.3 & 12.53 & 1.23 & 45,506 & 0 & 5 & 0 & 0 \\
\hline 15 & 50 & 83.99 & 84.08 & 0.09 & 64,334 & 0 & 6 & 0 & 0 \\
\hline 23 & 123 & 12.29 & 12.38 & 0.09 & 35,719 & 0 & 4 & 0 & 0 \\
\hline 49 & 236 & 9.22 & 9.29 & 0.07 & 36,328 & 0 & 5 & 0 & 0 \\
\hline 57 & 267 & 26.22 & 26.39 & 0.17 & 61,844 & 0 & 7 & 0 & 0 \\
\hline 60 & 3 & 0.25 & 0.37 & 0.12 & 12,061 & 0 & 2 & 0 & 0 \\
\hline 61 & 1 & 189.32 & 189.39 & 0.07 & 61,616 & 0 & 7 & 0 & 0 \\
\hline 62 & 6608 & 2.11 & 2.19 & 0.08 & 17,154 & 3 & 4 & 0 & 0 \\
\hline 63 & 7 & 59.59 & 59.72 & 0.13 & 68,462 & 0 & 6 & 0 & 0 \\
\hline 64 & 1 & 192.61 & 192.69 & 0.08 & 19,674 & 0 & 3 & 0 & 0 \\
\hline 65 & 7 & 59.34 & 59.48 & 0.14 & 68,462 & 0 & 6 & 0 & 0 \\
\hline 68 & 267 & 16.74 & 17.4 & 0.66 & 104,628 & 0 & 8 & 0 & 0 \\
\hline 70 & 267 & 20.32 & 22.83 & 2.51 & 133,391 & 0 & 8 & 0 & 0 \\
\hline 71 & 123 & 9.15 & 9.95 & 0.8 & 31,403 & 0 & 4 & 0 & 0 \\
\hline 72 & 29 & 244.27 & 244.34 & 0.07 & 23,333 & 0 & 4 & 0 & 0 \\
\hline 74 & 123 & 12.29 & 12.38 & 0.09 & 35,719 & 0 & 4 & 0 & 0 \\
\hline 75 & 123 & 14.1 & 14.22 & 0.12 & 35,719 & 0 & 4 & 0 & 0 \\
\hline 76 & 1 & 188.98 & 189.04 & 0.06 & 61,616 & 0 & 6 & 0 & 0 \\
\hline 78 & 267 & 7.09 & 8.09 & 1 & 37,718 & 0 & 5 & 0 & 0 \\
\hline 81 & 7602 & 3.93 & 4.11 & 0.18 & 27,165 & 4.8 & 8 & 0 & 0 \\
\hline
\end{tabular}




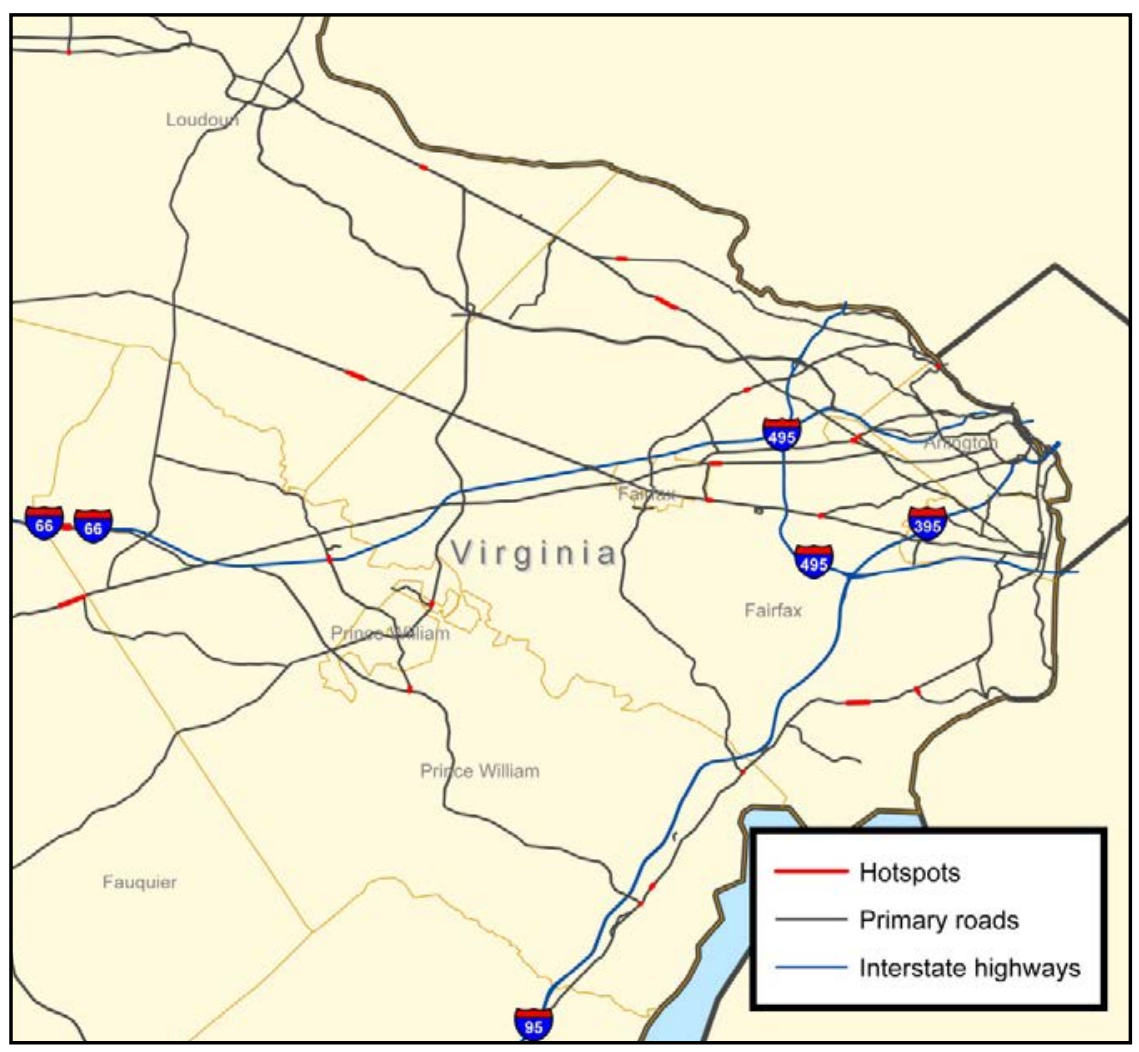

Figure 9.1. Top northern Virginia crash hot spots identified by VDOT.

of this effort is to query NDD in which the drivers passed through the locations. A list of the 20 hot spots had previously been identified by VDOT engineers. This list is based on injury and fatality rates and includes all crash types. The list of these locations was converted into a database table and imported into GIS. Again making use of the ability to join database tables with geospatial data, SQL queries were used to join the hot spots list with VDOT road centerline data to create a layer of polylines that corresponded to these 20 hotspot road segments. These queries were similar to those used in the two-part process to join the VDOT and HPMS data.
Figure 9.1 shows the resulting layer mapped with the primary and Interstate routes.

In Figure 9.1, the hot spots have been drawn thicker to make them visible. Initially, the polylines that represent the road network, and therefore hot spots, do not have a width. To give the hot spots width in the data, a GIS tool was used to generate buffers around the centerlines. Buffers of $200 \mathrm{ft}$ on both sides of the centerlines were used around these polylines, creating polygons to represent the hot spots. The result of buffering the polylines can be seen in a close-up view of two hot spots in Figure 9.2.

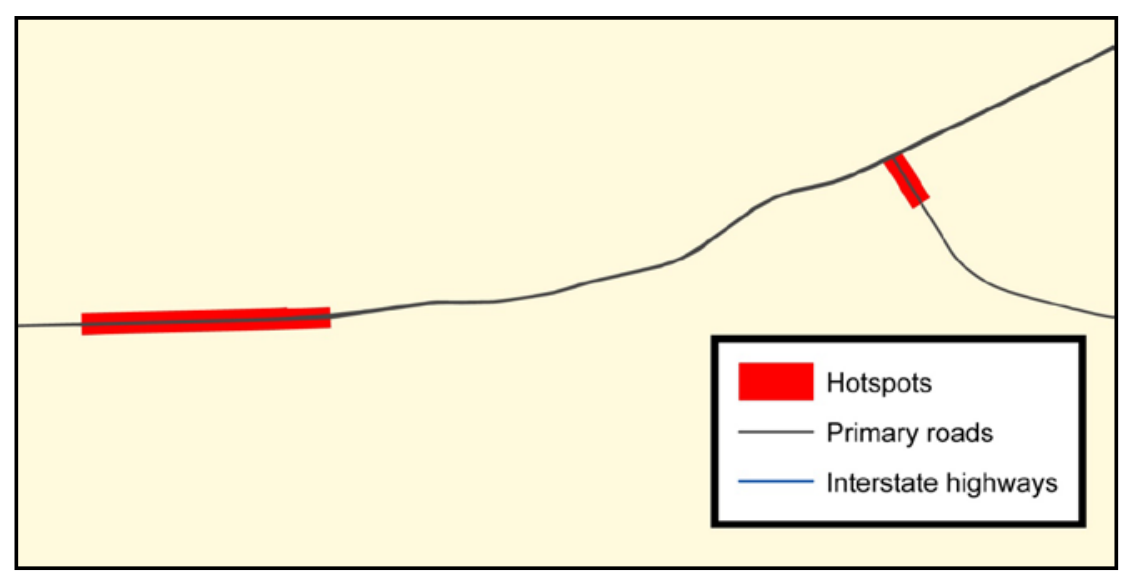

Figure 9.2. Buffers of $200 \mathrm{ft}$ created around hot spots. 
Once segments of interest such as these are identified by researchers, the next step is to locate naturalistic data describing the performance and behavior of drivers as they travel through the segments. To illustrate this step, a layer of breadcrumb trip points from 10 randomly selected trips by each vehicle in the 100-Car Study data set (1,000 trips total) was overlaid on the layer of hot spot polygons. This layer was created by using the ability to open a relational database table in ArcMap and have it plot the points defined by the table's latitude and longitude columns. The breadcrumb trip points that intersected with the polygons were used to identify trips where a vehicle passed through one of these 20 hot spots. A map of a selection of trip points through hot spots can be seen in Figure 9.3.

The figure illustrates primary, secondary, and local streets from VDOT centerline data. The blue lines are GPS breadcrumb trails from 100-Car trips. In the locations shown in dark brown, the trip is passing through one of the hot spots.

In addition to visualization of these epochs, a table was created that identifies all of the trips through the different hot spots, as well as all of the time series vehicle measures captured within the segment. This intermediate table can be further analyzed in a number of ways to identify contributing factors in crashes. The values can also be used to address video associated with the hot spot. To provide a small demonstration of what is found in these tables, box plots were created to describe the distributions of speeds found in the naturalistic data and maximum yaw values. In Figure 9.4, for each of the 20 hot spots, the speeds at which subject vehicles entered the hot spots are presented as a distribution. Outliers in the data are retained here. In the actual analyses, the outliers would be investigated further to determine if they were created, for example, by sensor errors or were true entry speed outliers. Cases in the tails of the distributions might be of interest as surrogates, particularly when speed appears to be a contributing factor in crashes for that hot spot.

Similarly, one might expect in challenging locations that exit speed would generally be lower than entrance speed. To illustrate how this might be explored in the data, the difference between segment exit speeds and entrance speeds was computed for each of the trips through the hot spots. This is provided in Figure 9.5.

Yaw is also a potential base surrogate measure related to roadway departure crashes. The maximum and minimum yaw observed as 100-Car participants traveled through the 20 hot spots is presented in Figure 9.6. Sign of the values was retained when creating the box plots. In these data, a positive yaw value indicates left-hand rotation of the vehicle around the vertical axis. Therefore, in general, the top plot in Figure 9.6 is indicating left-hand rotation and the bottom plot is indicating right-hand rotation.

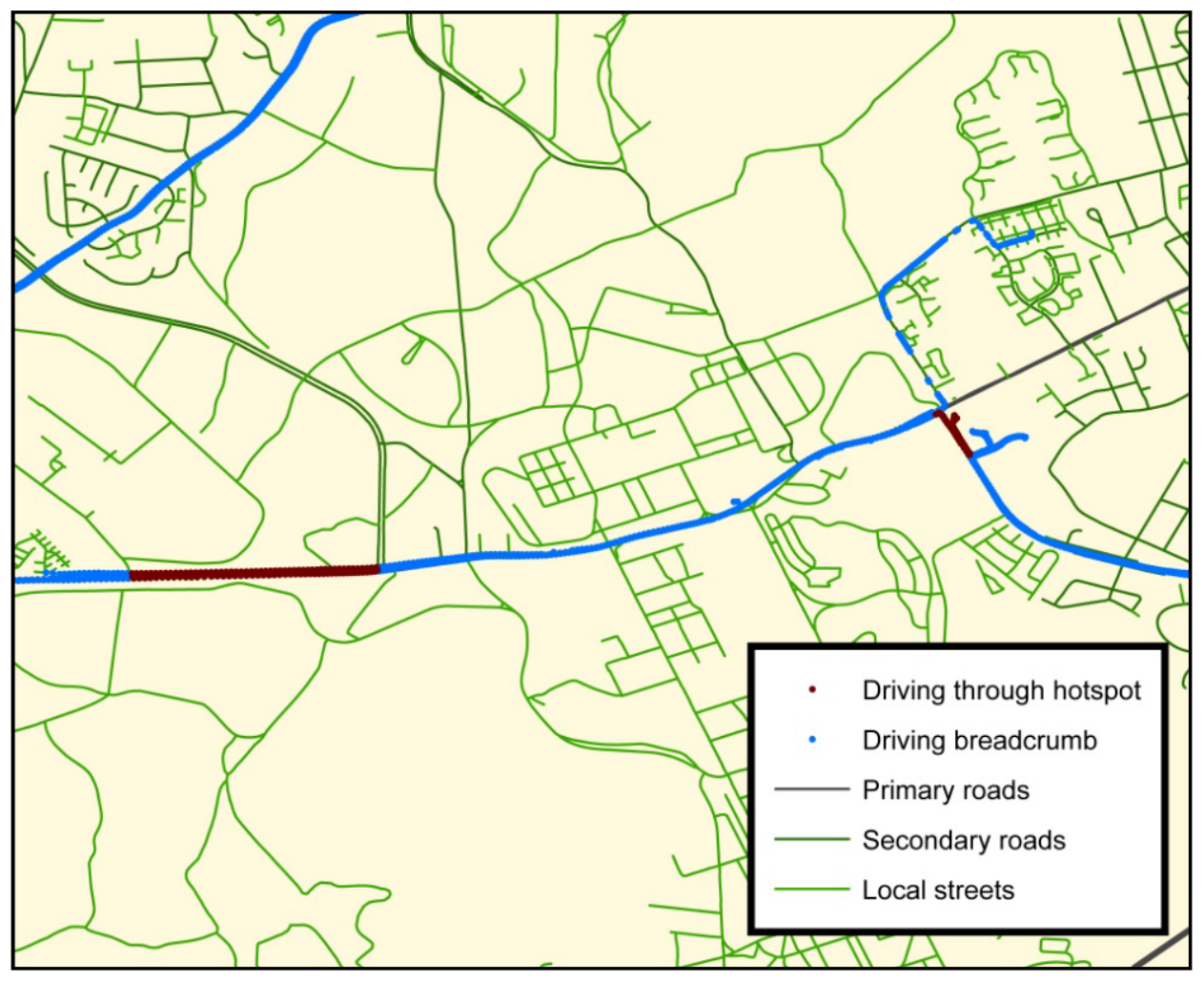

Figure 9.3. 100-Car trip points passing through hot spots. 


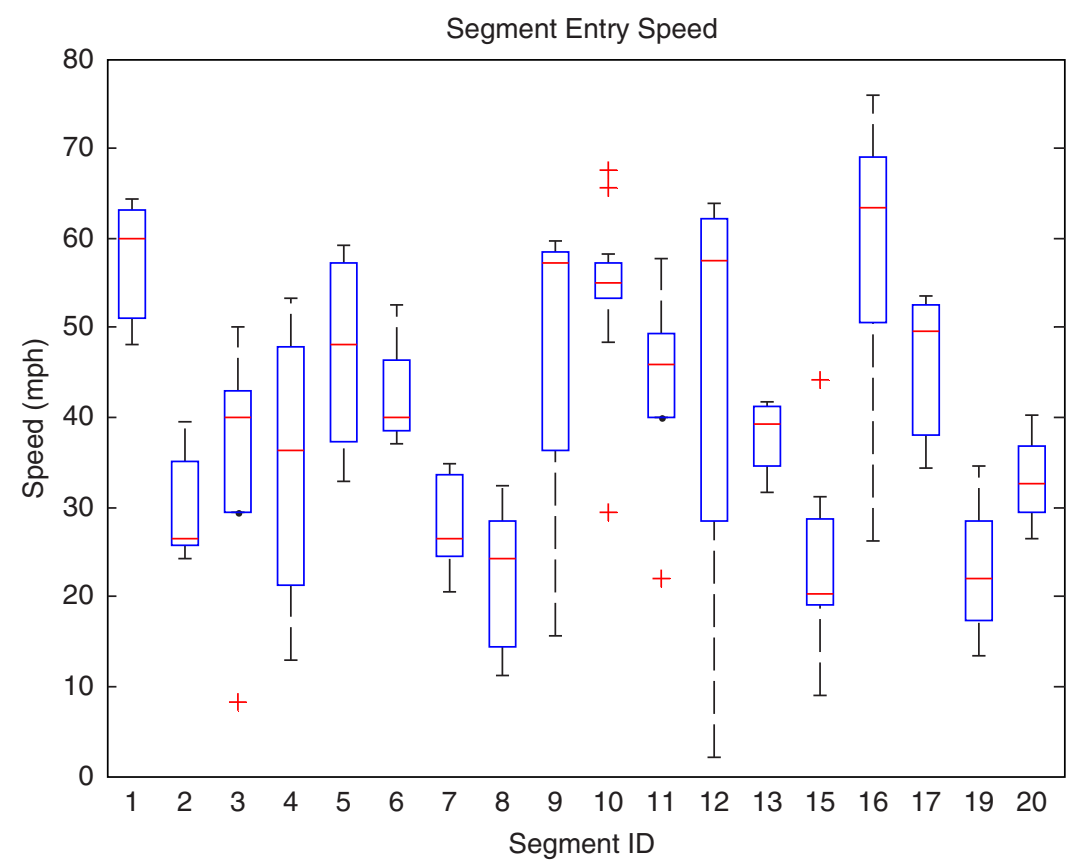

Figure 9.4. Distribution of vehicle speeds observed in naturalistic data when entering hot spot.

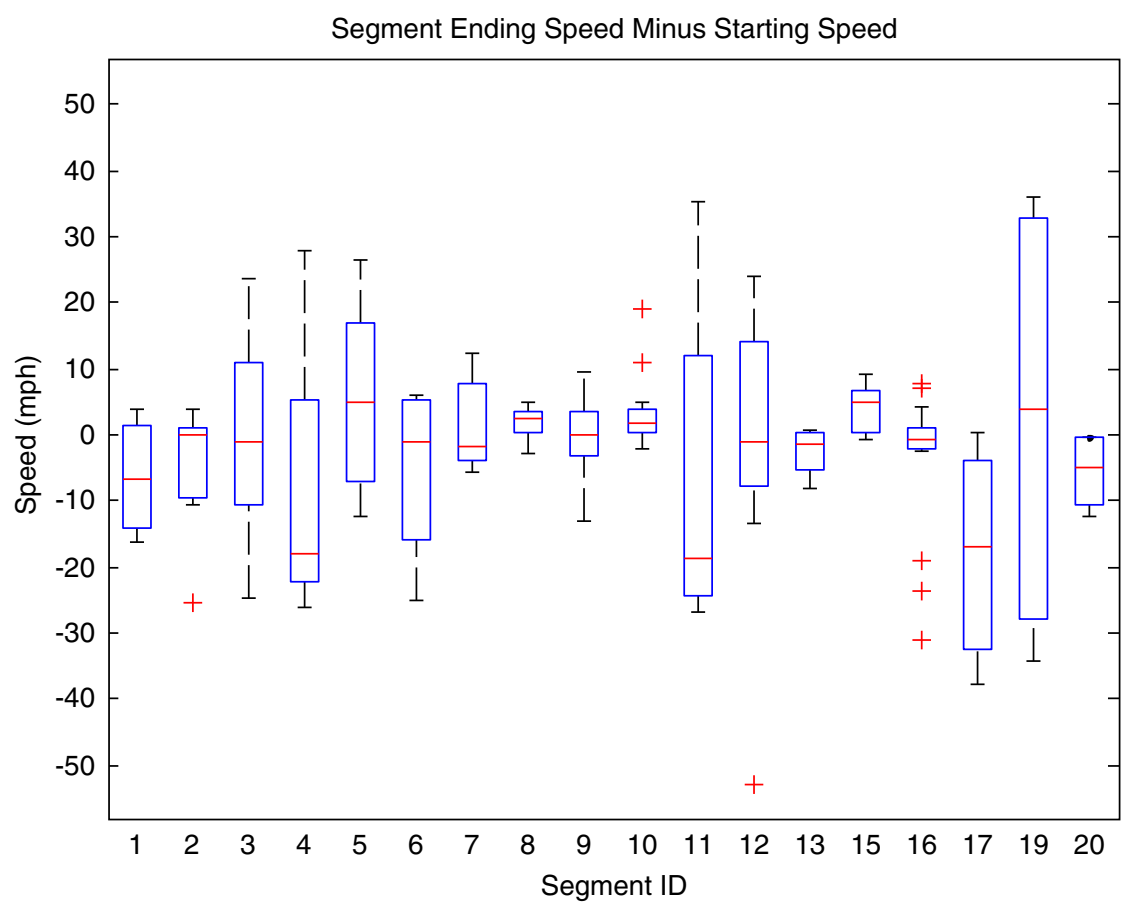

Figure 9.5. Difference in speed observed between entry into the hot spot segment and exiting the hot spot segment. Negative values indicate speed was reduced. 

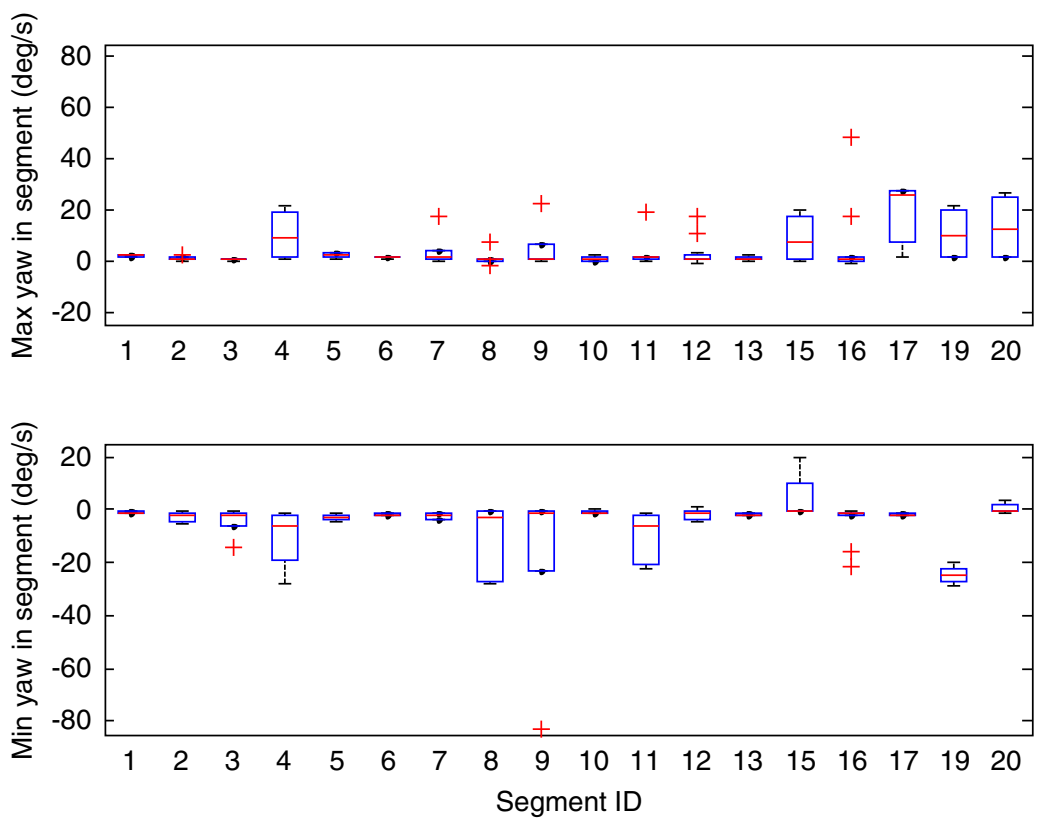

Figure 9.6. Distribution of maximum and minimum yaw observed in naturalistic data within the hot spots.

As with the speed measures, outliers may indicate cases of interest, such as a high-amplitude yaw event, or may indicate sensor anomalies.

\section{Identify Road Characteristics for Run-Off-Road Crashes}

For the VDOT crash records, exploration of road characteristics was illustrated in Table 9.2. Factors such as number of lanes, AADT, or road type may indicate explanatory factors in crashes. Similar associations can be made with events collected within a driving study (Table 9.3). The GPS data from

Table 9.3. Sample of a Roadway Explanatory Factor Collected from 100-Car Run-Off-Road Crashes

\begin{tabular}{|l|c|}
\hline Number of Lanes & $\begin{array}{c}\text { Number of 100-Car } \\
\text { Run-Off-Road Crashes }\end{array}$ \\
\hline 2 & 1 \\
\hline 3 & 1 \\
\hline 4 & 6 \\
\hline 5 & 3 \\
\hline 6 & 4 \\
\hline 7 & 2 \\
\hline 8 & 3 \\
\hline
\end{tabular}

the 100-Car Study run-off-road crashes were plotted in a layer in GIS, with the layer created from the joined VDOT centerline data and HPMS data. The features in the two layers were then spatially joined. This process associated the crashes from the 100-Car Study with the road segments where they occurred. The resultant geospatial data from the previously joined VDOT crash data road segments where the crashes occurred were also used here. After these spatial joins were performed, the road attributes contained in the VDOT road data and HPMS database were then available for analysis of crashes in both the crashes in the VDOT data and 100-Car Study crashes.

\section{Summary}

This chapter has demonstrated the viability of exploring segment crash rates as well as naturalistic data associated with high crash-rate locations using geospatial techniques. As found with the Michigan data, VDOT crash statistics can be joined with VDOT roadway segment data to identify, for example, the number of crashes on a segment. These data can then be joined with driver and vehicle measures collected in NDD. Integration of the data in this manner provides considerable power for identifying crash explanatory factors related to the driver, the environment, the vehicle, or the roadway.

The GIS methods provide a powerful tool for locating epochs of interest within naturalistic data. As with many of 
the tools for investigating these data, implementing the methods discussed here requires some GIS-specific understanding. These tools will be new for many researchers. An introductory workshop or clear documentation of procedures would be valuable.

When small data sets were processed in this manner, manual processes worked well. At the scale of the SHRP 2 study, geospatial analyses will require automated processes that can be running continuously or near continuously to generate the intermediate tables within a reasonable time frame. The programming languages native to the Esri suite should be explored for scalability. In the event that these tools are not able to support rapid processing, a possible alternative is to use an integration of the Mathworks Mapping toolbox, SQLbased geospatial data types, and a computational cluster that can run code developed in both of these applications. 


\section{HAPTER}

\section{Conclusions}

In Chapter 2, three general research questions were stated, together with 14 more specific questions that were intended to guide this research. The aim, even in the most optimistic moments, was not to answer all questions in a single project but to develop and test methods that have a strong bearing on these questions.

The broad research questions were:

- Do single-vehicle road departure crashes occur only under conditions of disturbed control?

- Do naturalistic driving data contain measurable episodes of disturbed control?

- Do objective measures of disturbed control from naturalistic driving data, together with highway geometric factors, off-highway factors, and environmental factors, satisfy criteria for crash surrogate (i.e., are they related to actual (rashes)?

On the one hand, the SUR analysis and the analysis of YRE in Chapter 7 provide ample indication that episodes of disturbed control exist in the naturalistic driving data, and that these episodes can be related to crashes via highway variables. On the other hand, insufficient data exist for the direct inclusion of off-highway factors, and little or no investigation was made in the area of other environmental factors such as lighting and weather conditions. From the data analyzed, it has not been possible to make any detailed progress on the first of the broad research questions: Do single-vehicle road departure crashes occur only under conditions of disturbed control? This lack of progress is not surprising, given that the data do not contain actual crashes. For the future SHRP 2 vehicle study, it should be a priority to establish this association. Given the very approximate estimation from Chapter 6 that around 15\% of road departures lead to actual crashes (at least on the road type considered), it should also be a priority to capture episodes of drift off the paved highway. In this way the preconditions for an expanded set of events might be used to analyze the problem, increasing the number of events and removing the factors that determining whether an actual impact occurs.

Turning next to the more specific research questions, significant progress has been made on the following research questions.

Question 1: What measures exist in naturalistic driving data that directly measure disturbed control?

The candidate surrogates LDW and TTEC provide some general measure of disturbed control, as response-only variables, while YRE appears to provide a more focused and control-related measure of this aspect.

Question 2: Are vehicle kinematic measures sufficient to identify disturbed control for risk measures in single-vehicle road departure crashes?

It has been shown that vehicle kinematics can provide a basis for risk analysis and surrogate validation. Whether this can be improved by using direct measurement of driver action has not, however, been analyzed.

Question 3: Are other driving control metrics necessary (in addition to vehicle kinematic measures) to identify disturbed control?

This question has not been resolved within the current data and time limitations of the project, but it is surely feasible in terms of some of the surrogates formulated but not analyzed in Chapter 4 (especially those relating to coherency or visual interruption).

Question 4: Are there measures of driving control performance in existing FOT data that depend on highway factors in a way that is consistent with single-vehicle road departure crash frequencies?

The prime success of the research presented relates to this research question. Through SUR analysis, it is certainly the case that measures of control performance can be related to crash data via the use of independent highway factors.

Question 9: What statistical tests are available to determine if the measures of driving control performance in naturalistic 
data and single-vehicle crashes depend on geometric features in a consistent way?

SUR analysis provides a partial answer to the question of what statistical tests are available.

Question 10: Can satisfactory crash risk predictions be made on the basis of vehicle/driver/highway information from naturalistic driving (e.g., via extreme value theory), or do additional roadside and environmental factors need to be introduced?

Extreme value theory suggests that a positive answer to Question 10 is also feasible, though in this case only a simple initial analysis was made. However, the potential benefits of introducing other roadside and environmental factors (in some form of composite surrogate) have not been tested.

Little progress has been made on the following research questions.

Question 5: Are there specific highway features that are associated with single-vehicle road departure crashes and specific driving control performance measures?

A small example of data exploration was presented for cases in which the right lane boundary was poorly defined or ambiguous, but it was not possible to find large numbers of such locations. While the optical lane tracker has some potential to be used in this way, a small attempt to conduct such an analysis did not prove fruitful. The lane tracker, when used to track broken or missing boundaries in this way, simply did not create reliable results (checking video revealed no useful patterns-i.e., the lane tracker detection states for the UMTRI NDD were not reliable predictors of road marking and lane conditions).

Question 6: Can roadside factors (e.g., locations of poles, trees, bridge abutments, and side slopes) be coupled to naturalistic driving data?

Question 7: Does the coupling of roadside factors to naturalistic driving data improve correlation with actual crashes?

Question 8: Can general descriptors of roadside environments be used in this coupling (e.g., tree density, proportion of side slope steeper than 4 to 1), or do we have to be more specific about location of roadside obstacles?

For Questions 6 to 8, relating to roadside factors, progress was not possible because of the lack of objective data for features such as cross-slope, trees, and poles. This lack of data was the most disappointing aspect of the study, especially since it is quite possible that SUR analysis could be improved with these additional factors used in the crash model (though naturally excluded in the surrogate model).

There was again little objective data directly available relating to driver state for the questions concerned with driver factors.

Question 11: Is the pattern of driving control performance different for the same driver when distracted versus not distracted (e.g., on a cell phone or not on a cell phone)?
Question 12: Can various driver states (e.g., drowsy, aggressive, distracted, engaged) be identified from naturalistic driving data?

Question 13: Can driving control performance for various states be categorized more simply (i.e., good and bad, or risky and nonrisky)?

Question 14: Is there a difference in the driving control performance of good and bad drivers (or risky and nonrisky drivers) at locations with geometric features associated with high single-vehicle crash frequency?

Some progress has been hinted in analysis of the YRE criterion. In Chapter 7, the figures presented indicate that a coherent interaction between yaw rate, yaw rate critical limits, and steering intervention is at the heart of active and effective lane-keeping control. Further work on this is necessary, but if successful, it may make active monitoring of driver gaze less critical to the assessment of driver attention state during driving. The cell phone study described in Chapter 8 under Cell Phone Use was unable to show any systematic differences between with cell phone and without cell phone, but a number of confounding factors may exist (especially in terms of the choice of time to speak on the phone). Again, a more sensitive measure of lane keeping - one based around coherency rather than simply on error magnitudes-may again be helpful. Thus there are suggestions that questions on detection and categorization of driver state (Questions 12 and 13) may be achievable. It should be noted, however, that this study relied on lateral control measures, and it is certainly the case that further analysis based on combined longitudinal and lateral control would be more complete in addressing those two questions. In this study, no analysis was considered in relation to interactions between driver types and highway risk features (Question 14). In the future it is quite possible to include such factors in the SUR analysis.

Turning to other aspects of the study, in Chapter 2 under Data Quality and Validation, the research team posed a number of questions about data availability and quality. According to Chapter 9, it is shown that the data types and quality are not unique to Michigan data. The Virginia DOT maintains spatially referenced data for crash and highway factors, and the SHRP 2 naturalistic driving study will provide many of the variable contained in the UMTRI NDD, expanded in some areas (such as head pose estimation) though reduced in other areas (such as number and location of radar units). Most, if not all, of the analysis conducted here with Michigan data is also feasible with Virginia data, though resources were too limited to engage in full replication studies.

In the SUR analysis it was recognized that a novel approach should be taken to exposure, with event rates based on segment traversals. In refining this idea, directional segments were defined, but consideration of curves made it clear that 
segment definitions were in need of further revision. There is a basic need for homogeneity in the segment with respect to the highway factors of interest. As such, HPMS segmentation is not generally appropriate, so in the future it makes good sense for safety researchers to define their own segment boundaries. Again, time was not sufficient to carry this analysis through, but it is clearly an important insight into how best to conduct future SUR analysis.
A final conclusion relates to the orthogonal studies presented in Chapter 8 and also the data analysis presented in Chapter 9: the combination of naturalistic, crash, and highway data provides such a rich data resource for research that it is relatively easy to develop new and innovative analysis methods specific to the data types. Therefore, making these data types available to independent researchers in the future seems certain to spawn new ideas and gather new insights. 


\section{References}

Agresti, A. 2002. Categorical Data Analysis, 2nd ed. Wiley-Interscience, New York.

Bauer, K. M., and D. W. Harwood. 1997. Statistical Models of At-Grade Intersection Accidents. Report FHWA-RE-96-125. U.S. Department of Transportation.

Bitzel, I. F. 1957. Accident Rates on German Expressways in Relation to Traffic Volumes and Geometric Design. Roads and Road Construction, Vol. 35, No. 409, pp. 18-20.

Brodsky, H., and A. S. Hakkert. 1988. Risk of a Road Accident in Rainy Weather. Accident Analysis and Prevention, Vol. 20, No. 2, pp. 161-176.

Caird, J., C. Scialfa, G. Ho., and A. Smiley. 2004. Effects of Cellular Phones on Driving Behavior and Crash Risk: Results of Meta-analysis. Edmonton, Alberta, Canada: AMA/CAA.

Chang, S., and T. J. Gordon. 2008. A Flexible Hierarchical Model-Based Control Methodology for Vehicle Active Safety Systems. Vehicle System Dynamics, Vol. 46, pp. 63-75.

Charlton, J., S. Koppel, M. O’Hare, D. Andrea, G. Smith, B. Khodoa, J. Langford, M. Odell, and B. Fildes. 2004. Influence of Chronic Illness in Crash Involvement of Motor Vehicle Drivers. Report 213. Monash University Accident Research Center, Victoria, Australia.

Chen, L. K., and A. Ulsoy. 2001. Identification of a Driver Steering Model and Model Uncertainty, from Driving Simulator Data. Journal of Dynamic Systems, Measurement, and Control, Vol. 123, No. 4, pp. 623-629.

Cirillo, J. A., S. K. Dietz, and R. L. Beatty. 1969. Analysis and Modeling of Relationships Between Accidents and the Geometric and Traffic Characteristics of the Interstate System. U.S. Department of Transportation.

Cirillo, J. A. 1970. The Relationship of Accidents to Length of SpeedChange Lanes and Weaving Areas on Interstate Highways. Highway Research Record 312, HRB, National Research Council, Washington, D.C., pp. 17-32.

Cleveland, D. E., L. P. Kostyniuk, and K. L. Ting. 1984. Geometric Design Element Groups and High-Volume Two-Lane Rural Highway Safety. Transportation Research Record 960, TRB, National Research Council, Washington, D.C., pp. 1-13.

Cleveland, D. E., L. P. Kostyniuk, and K. L. Ting. 1985. Design and Safety on Moderate-Volume Two-Lane Roads. Transportation Research Record 1026, TRB, National Research Council, Washington, D.C., pp. 51-61.

Coles, S. 2001. An Introduction to Statistical Modeling of Extreme Values. Springer, Berlin.

Comalli, P. E., S. Wapner, and H. Werner. 1962. Interference Effects of Stroop Color-Word Test in Childhood, Adulthood, and Aging. Journal of Genetic Psychology, Vol. 100, pp. 47-53.
Courage, C., P. Milgram, and A. Smiley. 2000. An Investigation of Attentional Demand in a Simulated Driving Environment. Proc., IEA 2000/ HFES 2000 Congress, EHES, Santa Monica, Calif., pp. III-336-III-339.

Dart, O. K., and L. Mann, Jr. 1970. Relationship of Rural Highway Geometry to Accident Rates in Louisiana. Highway Research Record 312, HRB, National Research Council, Washington, D.C., pp. 1-16.

David, N. A., and J. R. Norman. 1975. Motor Vehicle Accidents in Relation to Geometric and Traffic Features of Highway Intersections. Report FHWA-RD-76-129. U.S. Department of Transportation.

Dingus, T. A., S. G. Klauer, V. L. Neale, A. Peterson, S. E. Lee, J. Sudweeks, M. A. Perez, J. Hankey, D. Ramsey, S. Gupta, C. Bucher, Z. R. Doerzaph, J. Jarmeland, and R. R. Knipling. 2006. The 100-Car Naturalistic Driving Study, Phase II-Results of the 100-Car Field Experiment. Report DOT-HS-810-593. National Highway Traffic Safety Administration, U.S. Department of Transportation.

Di Stefano, M., and W. Macdonald. 2003. Assessment of Older Drivers: Relationships Among On-Road Errors, Medical Conditions and Test Outcome. Journal of Safety Research, Vol. 34, pp. 415-429.

Dunlap, D. F., P. S. Fancher, R. E. Scott, C. C. McAdam, and L. Segal. 1978. NCHRP Report 184: Influence of Combined Highway Grade and Horizontal Alignment on Skidding. TRB, National Research Council, Washington, D.C.

Eby, D. W., and L. J. Molnar. 1998. Matching Traffic Safety Strategies to Youth Characteristics: A Literature Review of Cognitive Development. Report 808 927. National Highway Traffic Safety Administration, U.S. Department of Transportation.

Eby, D. W., and L. P. Kostyniuk. 2004a. Estimating Driving Task Demand from Crash Probabilities: A Review of the Literature and Assessment of Databases. Safety Vehicles Using Interface Technology Final Report Task 2A. Volpe National Transportation System Center, U.S. Department of Transportation, Cambridge, Mass.

Eby, D. W., and L. P. Kostyniuk. 2004b. Distracted-Driving Scenarios: An Analysis of 2001 Crashworthiness Data System (CDS) Data. SAVE-IT/ University of Michigan Transportation Research Institute, Ann Arbor, Mich.

Federal Highway Administration. 1982. Synthesis of Safety Research Related to Traffic Control and Roadway Elements. Report FHWATS-82-232. U.S. Department of Transportation.

Federal Highway Administration. 2003. Interactive Highway Safety Design Model. www.tfhrc.gov/safety/ihsdm/ihsdm.hlm. Accessed May 2003.

Garber, N. J., and R. Gadiraju. 1990. Factors Influencing Speed Variance and Its Influence on Accidents. Transportation Research Record 1213, TRB, National Research Council, Washington, D.C., pp. 64-71. 
Getis, A., and J. K. Ord. 1996. Local Spatial Statistics: An Overview. In Spatial Analysis: Modelling in a GIS Environment (P. Longley and M. Batty, eds.), Geoinformation International, Cambridge, United Kingdom, pp. 261-277.

Glennon, J. C., T. R. Neuman, and J. E. Leisch. 1986. Safety and Operational Considerations for Design of Rural Highway Curves. Report FHWA-RD-86/035. U.S. Department of Transportation.

Godthelp, H. P. 1986. Vehicle Control During Curve Driving. Human Factors, Vol. 28, pp. 211-221.

Godthelp, H. P., P. Milgram, and G. Blaauw. 1984. The Development of a Time-Related Measure to Describe Driving Strategy. Human Factors, Vol. 26, pp. 257-268.

Golob, T. F., and W. W. Recker. 1987. An Analysis of Truck-Involved Freeway Accidents Using Log-Linear Modeling. Journal of Safety Research, Vol. 18, pp. 121-136.

Gordon, T. J., and N. Magnuski. 2006. Modeling Normal Driving as a Collision Avoidance Process. Proc., 8th International Symposium on Advanced Vehicle Control (AVEC), Taipei, Taiwan.

Green, P. E., and D. F. Blower. February 2007. Potential Effectiveness of Signal Optimization for Various Corridors in Michigan. Report 2007-5-1. Transportation Safety Analysis Division, UMTRI.

Gumbel, E. J. 1958. Statistics of Extremes. Columbia University Press, New York.

Hall, J. W., and O. J. Pendleton. 1989. Relationships between V/C Ratios and Accident Rates. Report FHWA-HR-NM-88-02. U.S. Department of Transportation.

Halpern, D. 1992. Sex Differences in Cognitive Abilities, 2nd ed. Lawrence Erlbaum Associates, Hillsdale, N.J.

Hanna, J. T., T. E. Flynn, and L. T. Webb. 1976. Characteristics of Intersection Accidents in Rural Municipalities. Transportation Research Record 601, TRB, National Research Council, Washington, D.C., pp. 79-82.

Harwood, D. W., F. M. Council, E. Hauer, W. E. Hughes, and A. Vogt. 2000. Prediction of the Expected Safety Performance of Rural Two-Lane Highways. Report FHWA-RD-99-207. U.S. Department of Transportation.

Harwood, D. W. and A. D. St. John. 1985. Passing Lanes and Other Operational Improvements on Two-Lane Highways. Report FHWARD-85-028. U.S. Department of Transportation.

Hoerrey, W. J., and C. D. Wickens. 2004. The Impact of Cellular Phone Conversations on Driving: A Meta-Analytical Approach. Technical Report AHFD-04-02/GM-04-1. Human Factors Division, University of Illinois.

Ivey, D., L. Griffin, T. Newton, and R. Laden. 1981. Predicting Wet Weather Accidents. Accident Analysis and Prevention, Vol. 13, pp. 83-89.

Janke, M. K. 1994. Age-Related Disabilities That May Impair Driving and Their Assessment. Report RSS-94-156. California Department of Motor Vehicles, Sacramento.

Jonah, B. A. 1997. Sensation Seeking and Risky Driving: A Review and Synthesis of the Literature. Accident Analysis and Prevention, Vol. 29, pp. 651-665.

Jones, B. L., L. Janssen, and F. Mannering. 1991. Analysis of the Frequency and Duration of Freeway Accidents in Seattle. Accident Analysis and Prevention, Vol. 23, pp. 239-255.

Jovanis, P., and J. Delleur. 1981. Exposure-Based Analysis of Motor Vehicle Accidents. Accident Analysis and Prevention, Vol. 13, pp. 83-99.

Khattak, A. J., P. Kantor, and F. M. Council. 1998. The Role of Adverse Weather in Key Crash Types on Limited Access Roadways: Implications for Advanced Weather Systems. Presented at 77th Annual Meeting of the Transportation Research Board, Washington, D.C.

Kihlberg, J. K., and K. J. Tharp. 1968. NCHRP Report 47: Accident Rates as Related to Design Elements of Rural Highways. HRB, National Research Council, Washington, D.C.
Land, M., and J. Horwood. 1995. Which Parts of the Road Guide Steering? Nature, Vol. 377, pp. 339-340.

LeBlanc, D., J. R. Sayer, E. R. Winkler, S. Bogard, J. M. Devonshire, J. M. Medfford, M. Hagan, Z. Bareket, R. Goodsell, and T. Gordon. 2006. Road Departure Crash Warning System Field Operational Test: Methodology and Results, Vol. 1. Technical report prepared for National Highway Traffic Safety Administration, U.S. Department of Transportation.

Marottoli, R. A., E. D. Richardson, M. H. Stowe, E. G. Miller, L. M. Brass, L. M. Cooney, and M. E. Tinetti. 1998. Development of a Test Battery to Identify Older Drivers at Risk for Self-Reported Adverse Driving Events. Journal of the American Geriatric Society, Vol. 46, No. 5, pp. 562-568.

Matthews, L. R., and J. W. Barnes. 1988. Relation Between Road Environment and Curve Accidents. Proc., 14th ARRB Conference, Canberra, Australia.

Mourant, R. R., and Z. Ge. 1997. Measuring Attentional Demand in a Virtual Environments Driving Simulator. Proc., Human Factors and Ergonomics Society 41st Annual Meeting, HFES, Santa Monica, Calif., pp. 1268-1272.

National Highway Traffic Safety Administration. 2006a. Traffic Safety Facts 2005: Older Population. Report DOT-HS-810-622. U.S. Department of Transportation.

National Highway Traffic Safety Administration. 2006b. Traffic Safety Facts 2005. Report DOT-HS-810-631. U.S. Department of Transportation.

Oxley, J., G. Fidles, B. Corben, and J. Langford. 2006. Intersection Design for Older Drivers. Transportation Research Part F, Vol. 9 , pp. 335-346.

Parasuraman, R., and Greenwood, P. M. 1998. Selective Attention in Aging and Dementia. In The Attentive Brain (R. Parasuraman, ed.), MIT Press, Cambridge, Mass.

Raff, M. S. 1953. Interstate Highway-Accident Study. Highway Research Board Bulletin 74, HRB, National Research Council, Washington, D.C., pp. 18-43.

Roy Jorgensen and Associates, Inc. 1978. NCHRP Report 197: Cost and Safety Effectiveness of Highway Design Elements. TRB, National Research Council, Washington, D.C.

Satterwaite, S. P. 1976. An Assessment of Seasonal and Weather Effects of Road Accidents in California. Accident Analysis and Prevention, Vol. 8, pp. 87-96.

Sayer, J. R., J. M. Devonshire, and C. A. Flannagan. 2005. The Effects of Secondary Tasks of Naturalistic Driving Performance. Report UMTRI25-29. University of Michigan Transportation Research Institute, Ann Arbor, Mich.

Schoppert, D. W. 1957. Predicting Traffic Accidents from Roadway Elements of Rural Two-Lane Highways with Gravel Shoulders. Highway Research Board Bulletin 158, HRB, National Research Council, Washington, D.C.

Senders, J. W., A. B. Kristofferson, W. H. Levison, C. W. Dietrich, and J. L. Ward. 1967. The Attentional Demand of Automobile Driving. Highway Research Record 195, HRB, National Research Council, Washington, D.C., pp. 15-33.

Sexton, M. A., and G. Geffen. 1979. Development of Three Strategies of Attention in Dichotic Monitoring. Developmental Psychology, Vol. 15, pp. 299-310.

Shafer, M. A., R. Q. Brackett, and R. A. Krammes. 1995. Driver Mental Workloads as a Measure of Geometric Design Consistency for Horizontal Curves. Report 950706. Texas Transportation Institute, College Station. 
Shankar, V., F. Mannering, and W. Barfield. 1995. Effects of Roadway Geometrics and Environmental Factors on Rural Freeway Accident Frequencies. Accident Analysis and Prevention, Vol. 27, pp. 371-389.

Snyder, J. F. 1974. Environmental Determinants of Traffic Accidents: An Alternate Model. Transportation Research Record 486, TRB, National Research Council, Washington, D.C., pp. 11-18.

Stokes, R. W., and M. I. Mutabazi. 1996. Rate-Quality Control Method of Identifying Hazardous Road Locations. Transportation Research Record 1542, TRB, National Research Council, Washington, D.C., pp. 44-48.

Stutts, J. C., J. R. Stewart, and C. Martell. 1998. Cognitive Test Performance and Crash Risk in an Older Driver Population. Accident Analysis and Prevention, Vol. 30, pp. 337-346.

Toffin, D., G. Reymond, A. Kemeny, and J. Droulez. 2007. Role of Steering Wheel Feedback on Driver Performance: Driving Simulator and Modeling Analysis. Vehicle System Dynamics, Vol. 45, No. 4, pp. 375-388.

Trachtler, A. 2004. Integrated Vehicle Dynamics Control Using Active Brake, Steering, and Suspension Systems. International Journal of Vehicle Design, Vol. 36, No. 1, pp. 1-12.

Tsimhoni, O., and P. Green. 1999. Visual Demand of Driving Curves as Determined by Visual Occlusion. Presented at the Vision in Vehicles VIII Conference, Boston, Mass.

Tsimhoni, O., and P. Green. 2001. Visual Demand of Driving and the Execution of Display-Intensive In-Vehicle Tasks. Proc., Human Factors and Ergonomics Society 45th Annual Meeting, HFES, Santa Monica, Calif., pp. 1586-1590.

Tsimhoni, O., H. Yoo, and P. Green. 1999. Effects of Visual Demand and In-Vehicle Task Complexity on Driving and Task Performance as Assessed by Visual Occlusion. Report UMTRI-99-37. University of Michigan Transportation Research Institute, Ann Arbor, Mich.

Van der Horst, R., and H. Godthelp. 1989. Measuring Road User Behavior with an Instrumented Car and an Outside-the-Vehicle Video Observation Technique. Transportation Research Record 1213, TRB, National Research Council, Washington, D.C., pp. 72-81.

Versace, J. 1960. Factor Analysis of Roadway and Accident Data. Highway Research Board Bulletin 240, HRB, National Research Council, Washington, D.C., pp. 24-32.
Vogel, K. 2003. A Comparison of Headway and Time to Collision as Safety Indicators. Accident Analysis and Prevention, Vol. 35, No. 3 , pp. 427-433.

Walsh, J. M., J. J. de Gier, A. S. Christopherson, and A. G. Verstraete. 2004. Drugs and Driving. Traffic Injury Prevention, Vol. 5, pp. 241-253.

Wierwille, W. W., R. J. Hanowski, J. M. Hankey, C. A. Kieliszewski, S. E. Lee, A. Medina, A. S. Keisler, and T. A. Dingus. 2002. Identification and Evaluation of Driver Errors: Overview and Recommendations. Report FHWA-RD-02-003. FHWA, U.S. Department of Transportation.

Wilkinson, C., and H. Moskowitz. 2001. Polypharmacy and Older Drivers: Literature Review. Southern California Research Institute, Los Angeles, Calif.

Wood, J. 2002. Age and Visual Impairment Decrease Driving Performance as Measured on a Closed-Circuit. Human Factors, Vol. 44, No. 3, pp. 482-494.

Wooldridge, M., K. Bauer, P. Green, and K. Fitzpatrick. 1999. Comparison of Driver Visual Demand in Test Track, Simulator, and On-Road Environments. Presented at 79th Annual Meeting of the Transportation Research Board, Washington, D.C.

Wooldridge, M., K. Fitzpatrick, R. Koppa, and K. Bauer. 2000. Effects of Horizontal Curvature on Driver Visual Demand. Transportation Research Record: Journal of the Transportation Research Board, No. 1737, TRB, National Research Council, Washington, D.C., pp. 71-77.

Zegeer, C. V., and J. A. Deacon. 1987. Effects of Lane Width, Shoulder Width, and Shoulder Type on Highway Safety. State-of-the-Art Report 6: Relationship Between Safety and Key Highway Features-A Synthesis of Prior Research. Transportation Research Board, National Research Council, Washington, D.C.

Zegeer, C., R. Stewart, D. Reinfurt, F. Council, T. Newman, E. Hamilton, T. Miller, and W. Hunter. 1990. Cost Effective Geometric Improvements for Safety Upgrading of Horizontal Curves. Report FHWA-RD-90-021. U.S. Department of Transportation.

Zellner, A. 1962. An Efficient Method of Estimating Seemingly Unrelated Regressions and Tests for Aggregation Bias. Journal of the American Statistical Association, Vol. 57, pp. 348-368.

Zuckerman, M. 1994. Behavioral Expressions and Biosocial Bases of Sensation Seeking. Cambridge University Press, New York. 


\section{Literature Review: Crash Rates and Highway, Environmental, and Driver Factors}

The ability to predict the occurrence of crashes on roadways has been a challenge to the transportation profession since the early days of motorized transportation, and a large number of studied have been conducted to relate highway safety to highway design, environmental and driver characteristics. The literature on these studies is extensive. A sample of studies relevant to the current research is briefly reviewed.

\section{Highway Design Factors}

There are three primary geometric elements in highway design and, thus, also in highway characteristics which have been studied in relation to crashes. These are cross section, horizontal alignment, and vertical alignment. Note that there is extensive literature relating intersection characteristics and crashes. However, because this study is concerned with lane departure crashes that occur on segments, the review does not include studies concerned with intersection crashes.

\section{Cross Section}

The major elements of cross section include the number and width of lanes, presence and type of median, type and width of shoulders, and roadside features (e.g., side slope, clear zone, placement and types of roadside obstacles). The effects of cross-sectional elements on crash occurrence have been examined in many empirical studies. A classic early study by Schoppert (1957) examined crash occurrence on two-lane rural roads in Oregon and developed descriptive and predictive models using regression analysis. Schoppert found that vehicle crashes were directly related to traffic volume and certain features of the roadway, including lane and shoulder widths. He found that the crash rate increased with reduced cross-section width but reported that lane and shoulder widths did not serve as good predictors of the number of crashes. Versace
(1960), in analyzing Schoppert's data, recognized that roadway features were correlated with each other; that is, good crosssectional elements usually go together, and furthermore, good cross-sectional elements were usually found together with good alignment. These, he noted, are the result of road design and construction practices. Versace identified shoulder and lane widths as factors affecting crash occurrence but noted that their effects were not as important as that of traffic volume. Increased crash rates with decreased lane and shoulder widths were also reported by Dart and Mann (1970) and Roy Jorgensen and Associates, Inc. (1978).

Kihlberg and Tharp (1968) investigated the relationship between motor vehicle crashes and highway design elements using data from five states by analyzing crash counts on homogenous road segments of two- and multilane roadways. They found that number of lanes and median affect crash rates. The effect of the median, however, was not very marked and was found in only some of the states examined.

Cleveland, Kostyniuk, and Ting $(1984,1985)$ examined crash data for two-lane rural highway segments from 14 states by using statistical categorical techniques. They confirmed Versace's observation that good (or bad) geometric features were usually found together, and grouped cross-sectional features (lane width, shoulder width, side slope, ditch condition) that were usually found together in roads into a set of "geometric bundles" that varied from excellent to poor. An effect of the geometric bundles on crashes was found, but it was not as important as the effects of traffic volume and access density and the interactive effect of the geometric bundles and access density.

A study by Zegeer and Deacon (1987) quantified the effects of lane width, shoulder width, and shoulder type on highway crash experience based on the analysis of data for nearly $5,000 \mathrm{mi}$ of two-lane highway from seven states. The study controlled for many roadway and traffic features, including roadside hazard, terrain, and traffic volume. Lane width 
and shoulder type and width were found to be related to crash rates and could also be related to crash type. A crash prediction model was developed and used to determine the expected effects of lane and shoulder widening improvements on related crashes.

A large effort at the Federal Highway Administration (FHWA 1982; Cirillo, Dietz, and Beatty 1969; Cirillo 1970) investigated the effects of geometric and traffic parameters on crashes on the Interstate system. By using data from 24 states, regression models were developed for 19 model categories for various segments of the Interstate freeway system, including interchanges of the mainline roadway. The basic finding of these analyses concerning geometric elements, which included cross section, was that because the geometrics on Interstate roads are generally very good, their variations, when they occur, have little influence on crashes.

The Interactive Highway Safety Design Module (IHSDM) is a suite of software analysis tools developed by FHWA to evaluate safety and operational effects of geometric design decisions during the design process (FHWA 2003). The IHSDM consists of modules, among which is the Crash Prediction Module, which estimates the number and severity of crashes that could be expected on specified road segments based on its geometric design and traffic characteristics. The IHSDM includes an algorithm for predicting the crashes on two-lane rural roads (Harwood et al. 2000). The base model provides an estimate of the safety performance on a roadway or intersection for a set of assumed nominal conditions. The modification factors adjust the base model predictions to account for the effects on safety for roadway segments of various geometric and operational features. For cross-sectional elements, the base conditions are two $12-\mathrm{ft}$ lanes, paved $6-\mathrm{ft}$ shoulders, and a roadside characterized as marginally recoverable. The adjustment factors vary by traffic volume conditions. According to the IHSDM crash prediction algorithm, the effects of lane width and shoulder type and width at low traffic volumes are very limited, but become larger at traffic volumes of more than 2,000 vehicles per day. The IHSDM algorithm also includes the effects of passing lanes on crash rates on two-lane rural roads. Based on the work of Harwood and St. John (1985), the algorithm predicts a reduction of crash rates with the installation of short fourlane sections that allow passing. It is worth noting that the IHSDM algorithm is based on historical accident data, before-and-after studies, as well as expert judgment as the basis for the factor analysis, and is an excellent example of a classical analysis that provides a contrast to the work contained in the present project. Perhaps a future extension of the IHSDM algorithm will include further data from naturalistic driving, site-based trajectory analysis and analysis of spatially referenced data.

\section{Horizontal and Vertical Alignment}

Elements of horizontal alignment include degree and length of horizontal curve, presence of spiral or other transition curve, and the superelevation. Elements of vertical alignment include vertical lines or grades and vertical curves (sags and crests). In a study of two-lane and multilane rural roads from 15 states, Raff (1953) found that crash rates increased with the degree of curvature. On two-lane roads, the crash rate increased with curve frequency. Crash rates also increased with sight distance restrictions, which are primarily due to crest vertical curves. The study also found that grade alone did not have an effect on crash rates on tangent (straight) sections of road, but there was an increase in crash rates when both grade and horizontal curvature were present. Increased crash rates on combinations of grades and horizontal curves have also been reported by Bitzel (1957). Bitzel's data was from 25,500 crashes on 1,300 mi of German highways. However, Bitzel found that crash rates increased as grades increased. Kihlberg and Tharp (1968) found effects on crash rates of horizontal curves only for curves of 4 degrees or more and for grades of $4 \%$ or more.

The effect of horizontal curves on crashes was also investigated by Glennon, Neuman, and Leisch (1986). A database that included crash, geometric, and traffic data for two-lane rural highway segments from four states was developed for this study. There were more than 3,000 segments with horizontal curves and about 350 control tangent sections. Care was taken to select sites with uniform lane and shoulder conditions and to avoid influences of bridges, intersections, curbs and other nearby horizontal curves. Analysis of covariance methods were used to develop a model that related the number of crashes on curves to the traffic volume, degree of curve, and length of curve.

Matthews and Barnes (1988) analyzed curve crashes on $2,000 \mathrm{~km}$ of highways in New Zealand. They identified prior curvature (total number of curvature in the two km preceding the curve where a crash occurred) as having the largest effect on curve crash rates, followed by grades, and radius of curve. They also reported that crash risk was particularly high on short radius curves located at the end of long tangents and on steep down grades. Safety effects of horizontal curves on two-lane rural roads were studied by Zegeer et al. (1990), using 5 years of crash data from Washington State and detailed information on horizontal alignment [degree of curve, length of curve, curve direction, central angle, presence of spiral transition, roadside (recovery distance, roadside hazard rating), cross section (lane width, width and type of shoulder), and traffic volume]. In all, there were more than 12,000 crashes with an average of 0.22 crashes per curve. Statistical analysis revealed significantly higher curve crashes for sharper curves, narrower lane width on curves, lack of spiral transitions, and increased superelevation deficiency. 
All else being equal, higher traffic volume and longer curves were associated with significantly higher frequency of curve crashes.

Federal Highway Administration studies (FHWA 1982; Cirillo, Dietz, and Beatty 1969; Cirillo 1970) of the effects of geometric and traffic parameters on crashes on the Interstate system did not find a significant contribution of horizontal or vertical alignment on crash rates on freeways. However, a study by Dunlap et al. (1978) that examined the effects of horizontal and vertical curves on crash rates on the Pennsylvania and Ohio Turnpikes found no significant relationship between crash rates and grades and horizontal curves in Ohio, but there were increases in crash rates with increasing curvature of horizontal curves in Pennsylvania.

The IHSDM crash prediction algorithm for two-lane rural roads (Harwood et al. 2000) includes the effects of horizontal and vertical alignment. The base model provides an estimate of the safety performance on a tangent and flat road segment. The crash rate for long flat curves is only slightly higher than for tangent roadways. However, the crash rate increases with the sharpness and shortness of the curve. Spiral transitions to the curve mitigate the crash rates as does adequate superelevation. The algorithm also predicts an increase in crash rate for increases in grades at steeper grades.

\section{Environmental Factors}

For road departure crashes the main factors of additional interest are weather and traffic volumes. Of course other factors such as the presence of construction zones, access density, and intersection design all have a strong influence on crash rates and are of great general interest. Because the present focus is on road departure crashes, those factors will not be reviewed here.

\section{Weather}

Weather constitutes a set of environmental factors that can influence crash occurrence by increasing crash risk. Empirical evidence suggests that a wet road surface increases crash frequency (Jones, Janssen, and Mannering 1991) and that truck-involved freeway collisions increase on wet and icy road surfaces (Golob and Recker 1987). Many studies have investigated the impacts of adverse weather and road geometry on crashes (Khattak, Kantor, and Council 1998; Ivey et al. 1981; Jovanis and Delleur 1981; Snyder 1974; Brodsky and Hakkert 1988; Shankar, Mannering, and Barfield 1995). Satterwaitte (1976) analyzing California data, found a ratio of the number of crashes during $24 \mathrm{~h}$ when almost all crashes occurred in wet conditions to the number of crashes occurring in dry conditions to be 2.23 times. A study on Texas roadways (Ivey et al. 1981) found that wet crash frequency per mile increased with higher AADT, higher number of lanes, greater access density, higher proportion of time the road surface is wet, and higher traffic speed variation but lower speed and lower skid number (a measure of the surface friction). Shankar, Mannering, and Barfield (1995) found that crash frequency on an Interstate in the Snoqualmie Pass area of Washington State increased with a higher number of horizontal curves, higher maximum grades, higher frequency of rainy days, higher maximum daily snowfall in a month, interactions of maximum snowfall with grade, and with curves. Overall, the literature shows that crash frequencies are higher in adverse weather conditions because of reduced visibility and reduced road friction.

\section{Traffic Volume}

Relationships between crash occurrence and geometric and operational characteristics of roadways often use a measure of traffic volume as the exposure measure of crash occurrence. However, there is strong empirical evidence of relationships between crash rates and traffic volume, conditional on roadway characteristics (e.g., Schoppert 1957; Versace 1960; Cleveland, Kostyniuk, and Ting 1984, 1985; Hall and Pendleton 1989; Stokes and Mutabazi 1996; Garber and Gadiraju 1990). Schoppert's (1957) study found that crash rates increased with increases in vehicle volume. He also reported that crashes on low-volume roads did not appear to be related to any roadway feature. Versace (1960) found average daily traffic (ADT), which is a measure of traffic volume, to be the variable most highly related to crash occurrence. Cleveland, Kostyniuk, and Ting $(1984,1985)$ found the relationship between crashes on road segments and ADT to be nonlinear and the best predictor of crashes on twolane rural roads. They also found the interactive effects of access point density with ADT to be very important in predicting crashes. In roadways built for high-design speeds, such as freeways, traffic volume appears to be the most important predictor of crashes. Other studies that FHWA studied of crashes on the Interstate system (Cirillo, Dietz, and Beatty, 1969; Cirillo 1970) concluded that the traffic volumes and commercial traffic volumes were the main contributors to the explanation of the crashes on the Interstate system of roads. ADT was also found to be the most important variable in the relationship between traffic crashes and highway geometric design elements and traffic volumes on interchange ramps and speed-change lanes (Bauer and Harwood 1997).

The interactive effect of traffic volume on crash occurrence is built into the IHSDM crash prediction algorithm for twolane rural roads and intersections (Harwood et al. 2000). The effects of each of the design or operational features are given for different levels of ADT. 


\section{Driver Factors}

The driving task is also affected by the driver's characteristics and behaviors. The SHRP 2 Safety program has a strong focus on the safety impacts of human behavior, especially driver error and the interaction with relevant highway and other factors. Human factors research focuses on the specific ways in which the human characteristics and behaviors influence the driving process. This is useful in understanding the basic mechanisms of how, for example, highway factors interact with human factors in determining crash risk in any particular situation.

To help understand how human behaviors affect crash risk, Wierwille et al. (2002) proposed the following taxonomy of four major factors degrading driving performance:

1. Inadequate knowledge, training, and skill: A lack of understanding or misunderstanding of traffic laws, vehicle kinematics, driving techniques, or driver capabilities and limitations.

2. Impairment: Fatigue and drowsiness; use of alcohol and illegal drugs; illness; lack of or incorrect use of medication; disability or uncorrected disability.

3. Willful inappropriate behavior: Purposeful violation of traffic laws, regulation; aggressive driving; use of vehicle for intimidation or as a weapon.

4. Infrastructure, environmental problems: Traffic control device related; roadway related; alignment, sight distance, delineation; weather, visibility related.

State and national crash databases include some information on drivers including age, sex, and some contain information on driver impairment and distraction. For example, the National Automotive Sampling System (NASS) Crashworthiness Data System (CDS) contains variables on police-reported alcohol presence and distraction. Analysis of these data shows that in 2001 , police noted alcohol presence for about $5 \%$ of all crashinvolved drivers in the United States; $25 \%$ of crash-involved drivers were distracted in some way, including $5 \%$ who were sleepy or asleep. Among drivers involved in single-vehicle road departure crashes, alcohol presence was noted for 18\%; $40 \%$ were distracted in some way, including $21 \%$ who were sleepy or asleep (Eby and Kostyniuk 2004b).

Crash risk varies with age. Whether considered on a per mile basis or a per population basis, crash rates vary as a function of age (NHTSA 2006a). Safe and efficient driving requires the adequate functioning of a range of abilities including vision, perception, cognitive functioning and physical abilities, which tend to decline with age; the loss of efficiency in any of these functions can reduce performance and increase risk on the road (Janke 1994; Marottoli et al. 1998; Stutts et al. 1998; Wood 2002; Oxley et al. 2006). Chronic disease and medications also affect driving abilities and crash risk (e.g., Wilkinson and Moskowitz 2001; Charlton et al. 2004; Walsh et al. 2004). A study of driving errors by 533 older drivers (mean age 76.2 years), whose competence was in question (Di Stefano and Macdonald 2003) found that their most frequent errors were failure to turn their heads to look behind, nonuse of mirrors, problems maintaining lane position and lane keeping, and keeping up with traffic.

Driving performance problems and elevated crash risk among young drivers have been related to inexperience, lack of knowledge about factors such as driving situations, vehicle handling, problem solving, and decision-making strategies (Eby and Molnar 1998). Young drivers also tend to perceive less risk in driving than do older drivers and are poorer at identifying hazards when driving (Eby and Molnar 1998; Jonah 1997). Sensation seeking among young drivers has also been linked to unsafe driving behaviors and purposeful violation of traffic laws and regulations, crashes, and violations (Eby and Molnar 1998; Jonah 1997). High levels of sensation seeking [as measured on the sensation-seeking scale (Zuckerman 1994)] have been associated with drinking and driving in young driver populations. The relationship was much weaker for female drivers than male drivers, and appears to decline with age (Jonah 1997).

There are also other gender effects on the driving task and crash risk. Men and women differ in cognitive abilities (Halpern 1992), risk taking (Jonah 1997), and automobile crash rates (NHTSA 2006b). These differences suggest that men and women may also differ in driving performance in a given situation. Studies that have examined visual aspects of attention demand find that female drivers require more time viewing than do male drivers for the same situation (Courage, Milgram, and Smiley 2000; Tsimhoni and Green 1999; Tsimhoni and Green 2001; Tsimhoni, Yoo, and Green 1999).

\section{Infrastructure and Highway Features}

Horizontal curves and lane width are two roadway features associated with road departure crash risk. As such, human factors research includes many studies of visual demand (the amount of time drivers look at road) associated with these road features. Results of these studies indicate that curved roads require more visual demand than straight sections. Visual demand increased with decreasing lane width (e.g., Courage, Milgram, and Smiley 2000; Senders et al. 1967; Van der Horst and Godthelp 1989).

\section{Age}

Whether considered on a per mile basis or a per population basis, crash rates vary as a function of age (NHTSA 2006a). There is clear evidence of age effects in selective, divided, 
and sustained attention (Comalli, Wapner, and Werner 1962; Parasuraman and Greenwood 1998; Sexton and Geffen 1979).

Tsimhoni, Yoo, and Green (1999) and Tsimhoni and Green (2001) assessed the differential effects of young drivers (aged 21 to 28 years) and older drivers (aged 66 to 73 years) on visual demand (the proportion of time that a driver views the roadway over a segment of interest). Using the visual occlusion method in a driving simulator, they found that older drivers had significantly higher visual demand for straight roadway sections and three curves of different radii. In a similar study, Tsimhoni and Green (1999) investigated differences in visual demand among three age groups ( 18 to $24 ; 35$ to 54 ; 55 and older). Again, subjects drove both straight and curved sections of roadway. These researchers found increased visual demand by age group for all curve radii studied. On straight roadways, however, visual demand was significantly different only for the oldest age group. Thus, these studies show that there is a clear interaction among age, roadway type, and visual demand, and that for a given driving situation, older drivers will experience a greater visual demand than younger drivers.

\section{Gender}

Men and women differ in cognitive abilities (Halpern 1992), risk taking (Jonah 1997), and automobile crash rates (NHTSA 2006b). These differences suggest that men and women may also differ in driving performance in a given situation. Several studies have addressed the visual aspect of this issue (Courage, Milgram, and Smiley 2000; Tsimhoni and Green 1999; Tsimhoni and Green 2001; Tsimhoni, Yoo, and Green 1999). Generally these studies find that female drivers require more time viewing the road in a given situation than male drivers. For example, Courage, Milgram, and Smiley (2000) had subjects drive straight and curved roadways that varied in width. Over all conditions, they found that women required $8 \%$ more time viewing the road than did men. This significant difference was of the same magnitude as the effect of lane width found in the same study.

\section{Driving Speed}

Driving speed is not a human factor, but rather a human response to the situation urgency of the trip, to conditions on the road, and to conditions within the vehicle. It is a trade-off between reduced journey time and risk of crash or conviction (or other social impact), as well as a result of decisions made on comfort and workload issues. Speed is a result of driver choice and has a profound effect on crash risk.

Numerous investigations have shown that as velocity increases, the percent of time viewing the forward scene also increases (Courage, Milgram, and Smiley 2000; Godthelp, Milgram, and Blaauw 1984; Mourant and Ge 1997; Senders et al. 1967). Senders et al. studied velocities ranging from 5 to $75 \mathrm{mph}$ in an on-road occlusion study. They found a monotonic relationship between velocity and percent of time viewing the roadway. Mourant and $\mathrm{Ge}$ (1997) considered two velocities (20 and $60 \mathrm{mph}$ ). They found a 9-percentage-point increase in visual demand as velocity increased from 30 to $60 \mathrm{mph}$. In a study using similar speeds Courage, Milgram, and Smiley (2000) found slightly greater increases in visual demand. Thus, there is a clear relationship between the speed at which a driver is traveling and the visual demand of the driving situation.

\section{Visual Demands Associated with Highway Features}

Human factors research includes many studies of visual demand associated with road features, focusing on lane width and horizontal curves. Studies using the visual occlusion method have found that visual demand increased with decreasing lane width (Courage, Milgram, and Smiley 2000; Senders et al. 1967; Van der Horst and Godthelp 1989). For example, Courage, Milgram, and Smiley (2000) varied lane width in a mediumfidelity driving simulator. They found that as width varied from 3.7 to $2.7 \mathrm{~m}$ ( 12 to $9 \mathrm{ft}$ ), visual demand increased by $6 \%$. Thus, the effect of lane width is significant but not strong.

The visual demand of driving horizontal curves has also been studied extensively with the visual occlusion method (Courage, Milgram, and Smiley 2000; Godthelp 1986; Mourant and Ge 1997; Senders et al. 1967; Shafer, Brackett, and Krammes 1995; Tsimhoni and Green 1999; Tsimhoni, Yoo, and Green 1999; Wooldridge et al. 1999; Wooldridge et al. 2000). Generally, these studies show that drivers need more visual input for curves than for straight sections of roadway, indicating that curves require greater visual demand. Those studies that have systematically varied the features of curves (e.g., Shafer, Brackett, and Krammes 1995; Tsimhoni and Green 1999; Tsimhoni, Yoo, and Green 1999; Wooldridge et al. 2000) have found that visual demand (a) is inversely related to the radius of curvature, (b) does not vary much with deflection angle, (c) begins to rise at the end of the approach tangent and peaks at the beginning of the curve followed by a decline throughout the curve, and (d) was higher for S-curves than for broken-back curves, but the effect was weakened with a large separation between the curves. Note that a broken-back curve has two curves in the same direction, whereas an S-curve has two curves in opposite directions. Those findings held for both on-the-road and simulator studies.

Vertical curves and combinations of vertical and horizontal curves have not been studied in human factors research. The most likely reason for this is that driving simulators do not simulate vertical curves adequately and test courses are usually flat, and hence techniques such as visual occlusion cannot be easily applied. 


\section{ABPEEN DIX \\ Road Departure Crashes by Environmental and Driver Conditions}

The road departure crashes show different patterns of involvements than other crash types. Table B.1 shows the percentage distribution of selected environmental and driver conditions. The table shows distributions for road departure crashes (as defined here-that is, departing the road before a harmful event), nonroad departure crashes, and all crash types. The cells show the percentages, summing to $100 \%$ for each factor. Total involvements are shown at the bottom of the table. The percentages represent the 71,308 road departure involvements, 967,944 nonroad departure involvements, and 1,039,252 total involvements.

Road departure crash involvements are more evenly distributed across the week than are other crash involvements.
If anything, they tend to be more prevalent on Saturday and Sunday, with some ramping up from the work week on Friday. Tuesday has the lowest incidence of road departure crash involvements. This pattern is in marked contrast with nonroad departure crash involvements, which tend to have a higher incidence in the work week (Monday through Friday), with a decline on Saturday and the lowest incidence on Sunday.

Road departure crashes also show a relatively higher incidence of crash involvements in dark conditions, whether lighted or unlighted; on snowy or icy road surfaces; on roads in which access is fully controlled (such as Interstate highways); and in which the driver had been drinking or using illegal drugs. 
Table B.1. Environmental and Driver Factors in Road Departure Crashes and Other Crash Types

\begin{tabular}{|c|c|c|c|}
\hline \multirow[b]{2}{*}{ Factor } & \multicolumn{2}{|c|}{$\begin{array}{c}\text { Road Departure } \\
\text { Crash? }\end{array}$} & \multirow[b]{2}{*}{ All Crash Involvements } \\
\hline & No & Yes & \\
\hline \multicolumn{4}{|c|}{ Day of Week } \\
\hline Sunday & 9.2 & 16.1 & 9.6 \\
\hline Monday & 14.8 & 13.7 & 14.8 \\
\hline Tuesday & 15.2 & 12.5 & 15.0 \\
\hline Wednesday & 15.6 & 13.8 & 15.5 \\
\hline Thursday & 15.4 & 13.3 & 15.3 \\
\hline Friday & 17.6 & 14.2 & 17.4 \\
\hline Saturday & 12.1 & 16.5 & 12.4 \\
\hline Total & 100.0 & 100.0 & 100.0 \\
\hline \multicolumn{4}{|c|}{ Light Condition } \\
\hline Daylight & 71.5 & 48.1 & 69.9 \\
\hline Dawn/dusk & 5.1 & 5.5 & 5.1 \\
\hline Dark: Lighted & 15.4 & 22.3 & 15.9 \\
\hline Dark: Unlighted & 6.4 & 22.7 & 7.5 \\
\hline Other/unknown & 1.6 & 1.2 & 1.6 \\
\hline Total & 100.0 & 100.0 & 100.0 \\
\hline \multicolumn{4}{|c|}{ Road Surface Condition } \\
\hline Dry & 72.2 & 51.2 & 70.7 \\
\hline Wet & 18.6 & 19.7 & 18.7 \\
\hline Icy & 2.6 & 11.6 & 3.2 \\
\hline Snowy/slushy & 4.2 & 14.5 & 4.9 \\
\hline Other/unknown & 2.5 & 2.9 & 2.5 \\
\hline Total & 100.0 & 100.0 & 100.0 \\
\hline \multicolumn{4}{|c|}{ Roadway Access Control } \\
\hline No control & 67.9 & 55.9 & 67.1 \\
\hline Full control & 10.3 & 28.0 & 11.5 \\
\hline Other/partial & 3.1 & 2.3 & 3.0 \\
\hline Unknown & 18.7 & 13.9 & 18.4 \\
\hline Total & 100.0 & 100.0 & 100.0 \\
\hline \multicolumn{4}{|c|}{ Alcohol Use } \\
\hline None & 97.4 & 88.3 & 96.8 \\
\hline Used & 2.6 & 11.7 & 3.2 \\
\hline Total & 100.0 & 100.0 & 100.0 \\
\hline \multicolumn{4}{|c|}{ Drug Use } \\
\hline None & 99.7 & 98.9 & 99.6 \\
\hline Used & 0.3 & 1.1 & 0.4 \\
\hline Total & 100.0 & 100.0 & 100.0 \\
\hline$N=$ & 967,944 & 71,308 & $1,039,252$ \\
\hline
\end{tabular}




\section{Data Tables}

\begin{tabular}{|r|r|r|r|r|r|r|r|}
\hline \multicolumn{7}{|c|}{ Lateral Deviation (LDEV) } \\
\hline curve & freeway & rururb & rights & crash & $\begin{array}{r}\text { LDEV } \\
\text { event }\end{array}$ & \multicolumn{1}{c|}{ cexpose } & aexpose \\
\hline 1 & 1 & 1 & 3 & 102 & 286 & 262.37 & 5.46 \\
\hline 1 & 1 & 2 & 3 & 1,500 & 4,417 & $95,095.84$ & $1,957.44$ \\
\hline 1 & 2 & 1 & 1 & 77 & 137 & 101.39 & 8.04 \\
\hline 1 & 2 & 1 & 2 & 280 & 299 & 277.27 & 11.53 \\
\hline 1 & 2 & 1 & 3 & 58 & 27 & 7.96 & 0.24 \\
\hline 1 & 2 & 2 & 1 & 1,879 & 2,779 & $192,834.52$ & $4,391.00$ \\
\hline 1 & 2 & 2 & 2 & 827 & 1,417 & $10,907.50$ & 397.73 \\
\hline 1 & 2 & 2 & 3 & 798 & 1,687 & $19,569.09$ & 456.76 \\
\hline 2 & 1 & 1 & 3 & 153 & 335 & $1,224.56$ & 16.78 \\
\hline 2 & 1 & 2 & 3 & 86 & 250 & $1,437.40$ & 23.52 \\
\hline 2 & 2 & 1 & 1 & 123 & 190 & 661.86 & 34.93 \\
\hline 2 & 2 & 1 & 2 & 173 & 381 & 555.98 & 30.51 \\
\hline 2 & 2 & 1 & 3 & 52 & 71 & 29.37 & 0.94 \\
\hline 2 & 2 & 2 & 1 & 836 & 1,128 & $135,541.93$ & $2,842.46$ \\
\hline 2 & 2 & 2 & 2 & 361 & 464 & $8,788.65$ & 182.54 \\
\hline 2 & 2 & 2 & 3 & 255 & 382 & $3,708.49$ & 77.42 \\
\hline
\end{tabular}

Note: cexpose $=365$ * 5 * AADT * Section Length * 10-9; aexpose $=$ Traversals * Section Length * $10^{-4}$. 


\begin{tabular}{|l|r|r|r|r|r|r|r|}
\hline \multicolumn{7}{|c|}{ Lane Departure Warning (LDW) } \\
\hline curve & freeway & rururb & rights & crash & $\begin{array}{r}\text { LDW } \\
\text { event }\end{array}$ & cexpose & aexpose \\
\hline 1 & 1 & 1 & 3 & 102 & 14 & 262.3685 & 5.464514 \\
\hline 1 & 1 & 2 & 3 & 1,500 & 340 & $95,095.84$ & $1,957.439$ \\
\hline 1 & 2 & 1 & 1 & 77 & 32 & 101.3881 & 8.035152 \\
\hline 1 & 2 & 1 & 2 & 280 & 53 & 277.2732 & 11.53001 \\
\hline 1 & 2 & 1 & 3 & 58 & 2 & 7.964373 & 0.238431 \\
\hline 1 & 2 & 2 & 1 & 1,879 & 235 & $192,834.5$ & $4,390.998$ \\
\hline 1 & 2 & 2 & 2 & 827 & 110 & $10,907.5$ & 397.7283 \\
\hline 1 & 2 & 2 & 3 & 798 & 135 & $19,569.09$ & 456.7622 \\
\hline 2 & 1 & 1 & 3 & 153 & 21 & $1,224.556$ & 16.78393 \\
\hline 2 & 1 & 2 & 3 & 86 & 19 & $1,437.401$ & 23.52065 \\
\hline 2 & 2 & 1 & 1 & 123 & 19 & 661.8614 & 34.92711 \\
\hline 2 & 2 & 1 & 2 & 173 & 37 & 555.9811 & 30.50896 \\
\hline 2 & 2 & 1 & 3 & 52 & 7 & 29.36845 & 0.94122 \\
\hline 2 & 2 & 2 & 1 & 836 & 94 & $135,541.9$ & $2,842.457$ \\
\hline 2 & 2 & 2 & 2 & 361 & 54 & $8,788.652$ & 182.5388 \\
\hline 2 & 2 & 2 & 3 & 255 & 71 & $3,708.489$ & 77.42169 \\
\hline
\end{tabular}

Note: cexpose $=365 * 5 *$ AADT * Section Length * $10^{-9}$; aexpose $=$ Traversals * Section Length * $10^{-4}$.

\begin{tabular}{|l|r|r|r|r|r|r|r|}
\hline \multicolumn{7}{|c|}{ Time to Edge Crossing (TTEC) } \\
\hline curve & freeway & rururb & rights & crash & $\begin{array}{r}\text { TTEC } \\
\text { event }\end{array}$ & cexpose & aexpose \\
\hline 1 & 1 & 1 & 3 & 102 & 238 & 262.37 & 5.46 \\
\hline 1 & 1 & 2 & 3 & 1,500 & 3,357 & $95,095.84$ & $1,957.44$ \\
\hline 1 & 2 & 1 & 1 & 77 & 388 & 101.39 & 8.04 \\
\hline 1 & 2 & 1 & 2 & 280 & 426 & 277.27 & 11.53 \\
\hline 1 & 2 & 1 & 3 & 58 & 39 & 7.96 & 0.24 \\
\hline 1 & 2 & 2 & 1 & 1,879 & 2,990 & $192,834.52$ & $4,391.00$ \\
\hline 1 & 2 & 2 & 2 & 827 & 1,711 & $10,907.50$ & 397.73 \\
\hline 1 & 2 & 2 & 3 & 798 & 1,708 & $19,569.09$ & 456.76 \\
\hline 2 & 1 & 1 & 3 & 153 & 279 & $1,224.56$ & 16.78 \\
\hline 2 & 1 & 2 & 3 & 86 & 180 & $1,437.40$ & 23.52 \\
\hline 2 & 2 & 1 & 1 & 123 & 360 & 661.86 & 34.93 \\
\hline 2 & 2 & 1 & 2 & 173 & 454 & 555.98 & 30.51 \\
\hline 2 & 2 & 1 & 3 & 52 & 48 & 29.37 & 0.94 \\
\hline 2 & 2 & 2 & 1 & 836 & 1,345 & $135,541.93$ & $2,842.46$ \\
\hline 2 & 2 & 2 & 2 & 361 & 613 & $8,788.65$ & 182.54 \\
\hline 2 & 2 & 2 & 3 & 255 & 512 & $3,708.49$ & 77.42 \\
\hline
\end{tabular}

Note: cexpose $=365 * 5 *$ AADT * Section Length * 10-9; aexpose $=$ Traversals $*$ Section Length * $10^{-4}$. 


\section{APPEN D IX}

\section{Determination of Yaw Rate Error from Vehicle-Based Measurements}

As shown in Chapter 3, the critical case occurs when the turning radius $R$ satisfies the equation

$$
\sin \phi=\frac{d}{2 R}
$$

which is equivalent to the yaw rate condition

$$
r=\frac{2 U \sin \phi}{d}
$$

where $U$ is vehicle speed. Thus Equation D.2 defines the maximum yaw rate of the vehicle to avoid conflict with right boundary point $P$.

The idea is to use the vehicle itself, with known speed and yaw rate, to provide a reference for which to estimate the relative position of the lane boundary over time. From the variations in lateral lane position over time, the lane geometry is to be estimated, and variables such as $\phi$ and $d$ derived.

We need to estimate the azimuth offset $\phi_{0}$ for the direction of the velocity vector at the front wheel relative to the vehicle longitudinal axis (at low speed this is the steer angle, but in general it depends on the front axle cornering stiffness). In the case where steer angle and cornering stiffness is not available, a simple estimate can be made on the basis of general vehicle dynamics properties

$\tan \phi_{0}=\frac{r L}{U-r c}$

where $r$ is the yaw rate, $L$ is the wheelbase, $2 c$ is the front track, and $U$ is the instantaneous vehicle speed-this equation is based on the assumption of near-zero slip angle at the rear axle but is expected to be reasonably accurate. Simple adjustments are to be made to this equation when considering left boundary points.
Figure D.1 shows the modified geometry when boundary point $B$ is offset from the vehicle path. For simplicity assume a fixed preview time $T$ to the boundary point, and an approximately constant curvature for the path of the reference point from $Q$ to $P$. In the figure, $\phi$ is the azimuth angle to the boundary point $B$, while $\theta$ is now the critical azimuth angle corresponding to the motion from $Q$ to $P$. (Again, both angles are defined relative to the velocity vector, not the vehicle longitudinal axis.)

During the vehicle motion from $Q$ to $P$, the heading angle and direction of velocity vector $V$ change by $2 \theta$, so numerically integrating the yaw rate over the time interval $T$ we have

$\theta=\frac{1}{2} \sum_{i} r\left(t_{i}\right) \delta t$

The mean radius of turn, $R$, during the time interval can also be obtained from the yaw rate:

$\frac{2 \theta}{T}=\frac{\bar{U}}{R}$

where $\bar{U}$ is the mean vehicle speed during the interval, and both sides of this equation are estimates of the mean yaw rate during time interval $T$. Then, to determine $\phi$ we consider triangle $B P Q$ in Figure D.2. Angles at $P$ and $Q$ are known in terms of $\phi$ and $\theta$ and hence the angle at $B$ is given by

$\beta=180-(90-\theta+\alpha)=90+\theta-\alpha=90+2 \theta-\phi$

Then from the sine rule

$\frac{\sin \beta}{2 R \sin \theta}=\frac{\sin (\phi-\theta)}{s}$

which is a nonlinear implicit equation for $\phi$ in terms of other known variables. For normal highway driving we expect 


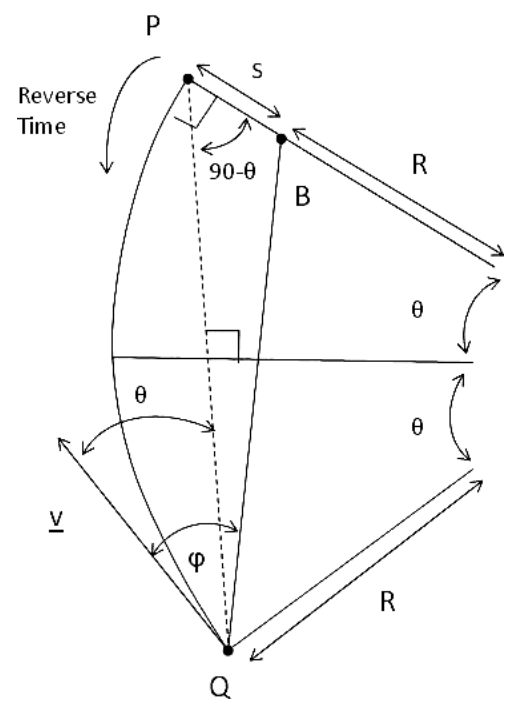

Figure D.1. Sketch of turning geometry for offset boundary point $\mathrm{B}$.

$\alpha=\phi-\theta$ to be sufficiently small (less than around $5^{\circ}$ ) to allow the approximation $\sin \alpha \approx \alpha, \cos \alpha \approx 1$. In this case,

$$
\begin{aligned}
\sin \beta & =\sin (\theta+(90-\alpha)) \\
& =\sin \theta \cos (90-\alpha)+\cos \theta \sin (90-\alpha) \\
& =\sin \theta \sin \alpha+\cos \theta \cos \alpha \\
& =\sin \theta \alpha+\cos \theta
\end{aligned}
$$

Substituting this into Equation D.6 then gives

$$
\frac{\sin \theta \alpha+\cos \theta}{2 R \sin \theta}=\frac{\alpha}{s}
$$

Hence

$\alpha\left(\frac{1}{s}-\frac{1}{2 R}\right)=\frac{1}{2 R \tan \theta}$

giving the approximate expression for $\phi$ (with all angles in radians)

$\phi=\theta+\frac{s}{(2 R-s) \tan \theta}$

Distance $d=|Q B|$ is also found from the geometry of Figure D.2:

$$
\begin{aligned}
d & =|Q P| \cos \alpha-|B P| \cos (90-\theta) \\
& \approx 2 R \sin \theta-s \sin \theta
\end{aligned}
$$

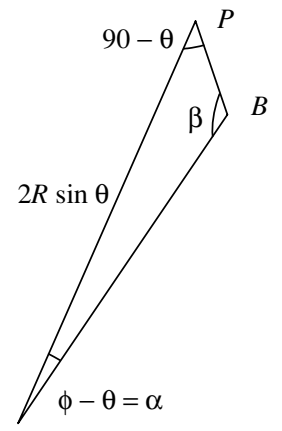

Figure D.2. Geometry to determine $\phi$.

And hence

$d=(2 R-s) \sin \theta$

Equations D.4, D.5, D.6a, and D.7 then determine all the relevant terms in the critical yaw rate expression

$r_{c}=\frac{2 U \sin \phi}{d}$

where $U$ is the instantaneous vehicle speed at $Q$, and now $r_{c}$ denotes the critical yaw rate.

Multiple calculations can be performed for point pairs $(P, Q)$ for values of $T$ in a range of say $0.5-2$ seconds, and the results referenced on the initial point $Q$. We are then interested in the minimum value of $r_{c}(Q)$ and its corresponding distance $d$ from $Q$. The yaw rate error (YRE) is then

$\operatorname{YRE}\left(t_{\mathrm{Q}}\right)=r\left(t_{\mathrm{Q}}\right)-r_{c} *(Q)$

where $r\left(t_{Q}\right)$ is the vehicle yaw rate at time $t_{Q}$, and $r_{c} *(Q)$ is the minimum critical yaw rate at $Q$.

A second YRE for left boundary points also has to be found, making similar calculations with relevant shift of reference point (to the outside of the left front tire) together with relevant sign changes.

The above equations are obtained for computing YRE but it is worth noting that, with a minor adjustment, they can be used to give a refined estimate of local road curvature from the onboard vehicle data (again assuming lane position, speed, and yaw rate are measured), thus removing the effects of vehicle lateral drift. The method is to estimate the critical yaw rate for a shifted point $P$ that has the same lateral offset as current point $Q$; thus replace $s=s(P)$ in the above, by $s^{\prime}=s(P)-s(Q)$. The critical yaw rate $r_{c}^{\prime}$ is then the yaw rate that maintains equal lane deviation over time interval $T$, and hence provides the radius of curvature $R_{e}$ (referenced at the right lane boundary). We obtain

$R_{e}=\frac{\bar{U}}{r_{c}^{\prime}}-s^{\prime}$ 


\section{TRB OVERSIGHT COMMITTEE FOR THE STRATEGIC HIGHWAY RESEARCH PROGRAM 2*}

CHAIR: Kirk T. Steudle, Director, Michigan Department of Transportation

\section{MEMBERS}

H. Norman Abramson, Executive Vice President (retired), Southwest Research Institute

Alan C. Clark, MPO Director, Houston-Galveston Area Council

Frank L. Danchetz, Vice President, ARCADIS-US, Inc.

Malcolm Dougherty, Director, California Department of Transportation

Stanley Gee, Executive Deputy Commissioner, New York State Department of Transportation

Mary L. Klein, President and CEO, NatureServe

Michael P. Lewis, Director, Rhode Island Department of Transportation

John R. Njord, Executive Director (retired), Utah Department of Transportation

Charles F. Potts, Chief Executive Officer, Heritage Construction and Materials

Ananth K. Prasad, Secretary, Florida Department of Transportation

Gerald M. Ross, Chief Engineer (retired), Georgia Department of Transportation

George E. Schoener, Executive Director, I-95 Corridor Coalition

Kumares C. Sinha, Olson Distinguished Professor of Civil Engineering, Purdue University

Paul Trombino III, Director, Iowa Department of Transportation

EX OFFICIO MEMBERS

Victor M. Mendez, Administrator, Federal Highway Administration

David L. Strickland, Administrator, National Highway Transportation Safety Administration

Frederick “Bud” Wright, Executive Director, American Association of State Highway and Transportation Officials

\section{LIAISONS}

Ken Jacoby, Communications and Outreach Team Director, Office of Corporate Research, Technology, and Innovation Management, Federal Highway Administration Tony Kane, Director, Engineering and Technical Services, American Association of State Highway and Transportation Officials Jeffrey F. Paniati, Executive Director, Federal Highway Administration

John Pearson, Program Director, Council of Deputy Ministers Responsible for Transportation and Highway Safety, Canada

Michael F. Trentacoste, Associate Administrator, Research, Development, and Technology, Federal Highway Administration

\section{SAFETY TECHNICAL COORDINATING COMMITTEE*}

CHAIR: Forrest M. Council, Senior Research Scientist, Highway Safety Research Center, University of North Carolina

\section{MEMBERS}

Timothy E. Barnett, State Safety Engineer, Alabama Department of Transportation

James A. Bonneson, Senior Principal Engineer, Kittelson and Associates

Richard K. Deering, President, RK Deering \& Associates, Inc.

Leanna Depue, Director, Traffic and Highway Safety Division, Missouri Department of Transportation

Bradley M. Estochen, State Traffic Safety Engineer, Minnesota Department of Transportation

Jurek Grabowski, Research Director, AAA Foundation for Traffic Safety

Jeffrey Greenberg, Senior Technical Leader, Ford Motor Company

Joanne Harbluk, Human Factors Specialist, Transport Canada

Brent Jennings, Highway Safety Manager, Idaho Transportation Department

Alan F. Karr, Director, National Institute of Statistical Sciences

Bani K. Mallick, Distinguished Professor, Department of Statistics, Texas AઐM University

John C. Milton, Director, Enterprise Risk \& Safety Management, Washington State Department of Transportation

Harlan J. Onsrud, Professor, School of Computing \& Information Science

Michael Perel, Safety Knowledge Engineer

Charles W. Reider, Chief Safety Engineer, Nevada Department of Transportation

David Shinar, Professor, Department of Industrial Engineering and Management, Ben Gurion University of the Negev

Alison Smiley, President, Human Factors North, Inc.

Thomas M. Welch, State Transportation Safety Engineer (retired), Office of Traffic and Safety, Iowa Department of Transportation

\section{AASHTO LIAISONS}

Kelly Hardy, Safety Program Manager, American Association of State Highway and Transportation Officials

Pam Hutton, SHRP 2 Implementation Manager, American Association of State Highway and Transportation Officials

Jim McDonnell, Program Director for Engineering, American Association of State Highway and Transportation Officials

\section{FHWA LIAISONS}

Monique Evans, Director, Office of Safety Technologies, Federal Highway Administration

Michael Griffith, Director, Office of Safety Integration, Federal Highway Administration

\section{AUTO INDUSTRY LIAISONS}

Michael Cammisa, Director, Safety, Association of Global Automakers

Scott Schmidt, Director, Safety and Regulatory Affairs, Alliance of Automobile Manufacturers

\section{EUROPEAN SAFETY LIAISON}

Fred Wegman, Managing Director, SWOV Institute for Road Safety Research, Netherlands

\section{FMCSA LIAISON}

Martin Walker, Chief, Research Division, Federal Motor Carrier Safety Administration

\section{NHTSA LIAISONS}

Richard Compton, Director, Office of Behavioral Safety Research, National Highway Traffic Safety Administration Tim Johnson, Director, Office of Human-Vehicle Performance Research, National Highway Traffic Safety Administration Seymour Stern, Team Leader, State Based Systems, National Highway Traffic Safety Administration

\footnotetext{
${ }^{\star}$ Membership as of April 2013.
} 


\section{Related SHRP 2 Research}

Development of Analysis Methods Using Recent Data (S01A)

Analysis of Existing Data: Prospective Views on Methodological Paradigms (S01B)

Evaluation of Data Needs, Crash Surrogates, and Analysis Methods to Address Lane Departure Research Questions Using Naturalistic Driving Study Data (S01E)

Design of the In-Vehicle Driving Behavior and Crash Risk Study (S05) Technical Coordination and Quality Control for Field Study (S06) Initial Analyses from the SHRP 2 Naturalistic Driving Study: Addressing Driver Performance and Behavior in Traffic Safety (S08) 